OSMIA la revue des apidologues

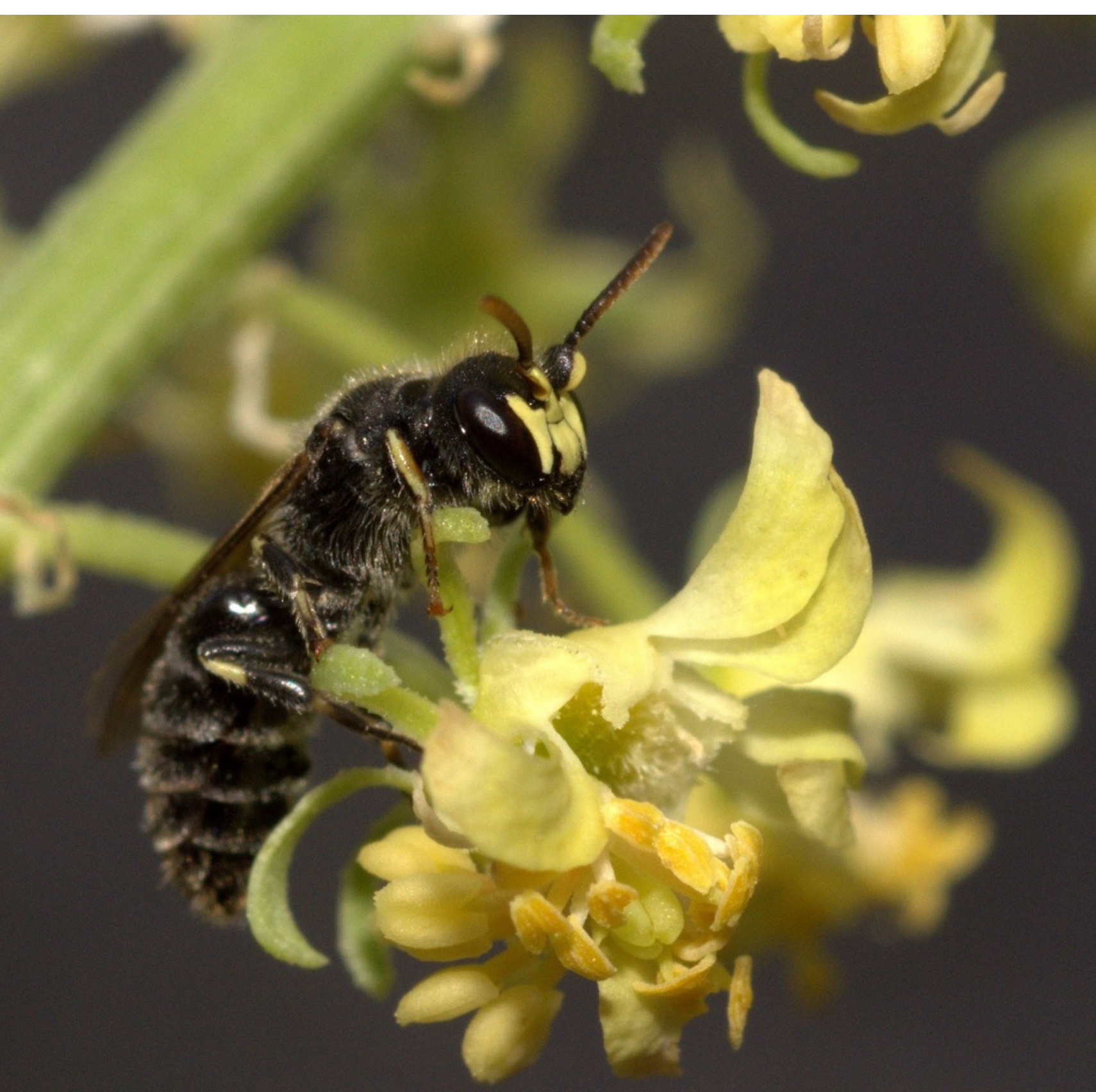

NUMÉRO 7 - ÉTÉ 2018 

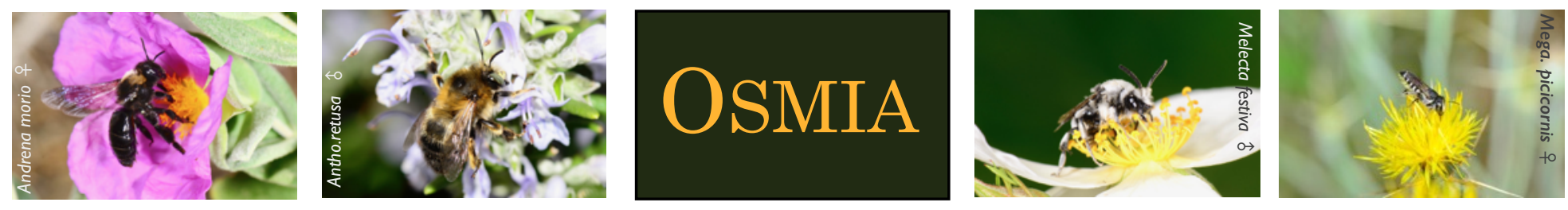

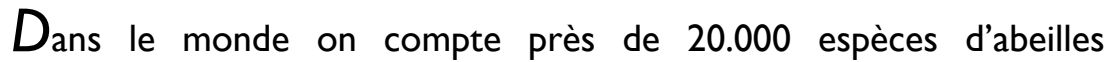
sauvages. Plus de 2000 en Europe et environ 970 en France. Les Apoïdes (les abeilles sensu lato) fascinent par leurs adaptations multiples, la diversité de leurs modes de vie et de leurs relations avec les plantes à fleurs. Les liens que les abeilles tissent avec les fleurs sont garant de la reproduction de plus de $80 \%$ d'entre elles, sur tous les continents, permettant leurs maintiens et leurs évolutions dans les écosystèmes.

Pour aller plus loin

$\star$ https://

fr.groups.yahoo.com/neo/

groups/apoidea-gallica/info

$\star$ www.oabeilles.net

\section{Pour partager}

* www.facebook.com/

Observatoire-des-

Abeilles-695II6727285I5

4 l

$\star$ https://twitter.com/

Obs Abeilles

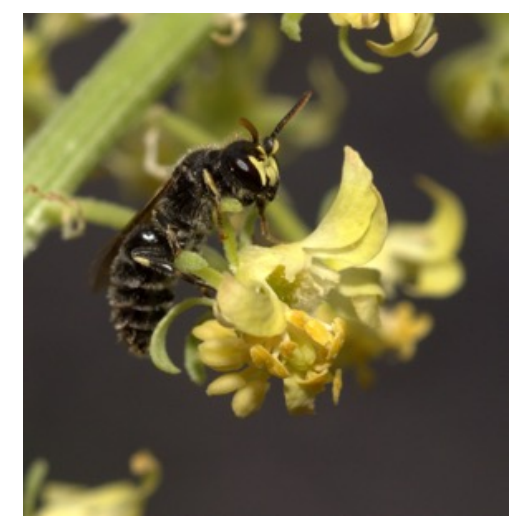

Photo de couverture

Mâle d'Hylaeus brachycephalus (Colletidae) sur Reseda sp. (Resedaceae). Juin 2017,Alpes du Sud, France. Matthieu Aubert.
Depuis maintenant plusieurs décennies, on observe un déclin, une perte de l'abondance et de la diversité des abeilles sauvages. Ainsi près de $10 \%$ des espèces seraient menacées d'extinction à l'échelle Européenne. En cause : la pression anthropique croissante et la perte d'habitats favorables à l'établissement et au maintien des populations d'abeilles sauvages. Le déclin associé de la flore sauvage et les pertes de rendement sur de nombreuses cultures font craindre une crise de pollinisation à l'échelle mondiale.

Pourtant, relativement à leur importance dans les écosystèmes, les abeilles sauvages sont peu connues et de nombreux aspects de leur biologie - distribution, morphologie, dynamique des populations, mode de vie restent encore mystérieux. C'est pourquoi s'est créé il y a maintenant une dizaine d'année le groupe Apoidea gallica, dans le but de rassembler les naturalistes, les scientifiques et les passionnés des abeilles sauvages. Les membres de ce groupe, originaires d'horizons divers, partagent le souhait d'approfondir les connaissances relatives à la biologie, à l'écologie et à l'évolution des Apoïdes, l'accent étant tout particulièrement mis sur l'observation de ces insectes dans leur milieu naturel et leur recensement en Europe de l'Ouest.

OSMIA est née de l'initiative de membres d'Apoidea gallica

Depuis 2008 des membres d'Apoidea gallica se sont regroupés au sein d'une association, L'Observatoire des Abeilles, qui continue de faire vivre OSMIA pour toute la communauté naturaliste et scientifique francophone avec la volonté que cette lettre de contact soit la plus riche possible. La diffusion d' OSMIA se fait gratuitement et au format PDF par l'intermédiaire du site web de l'Observatoire des Abeilles, et ce afin de permettre l'accès à la lettre de contact au plus grand nombre.

Nous espérons continuer à vous passionner et à vous faire vivre le monde des abeilles. Et chacun d'entre vous est le bienvenu pour s'inscrire sur la liste de diffusion Apoidea gallica, pour adhérer à l'Observatoire des Abeilles ou pour publier des articles dans notre journal à tous, OSMIA. 


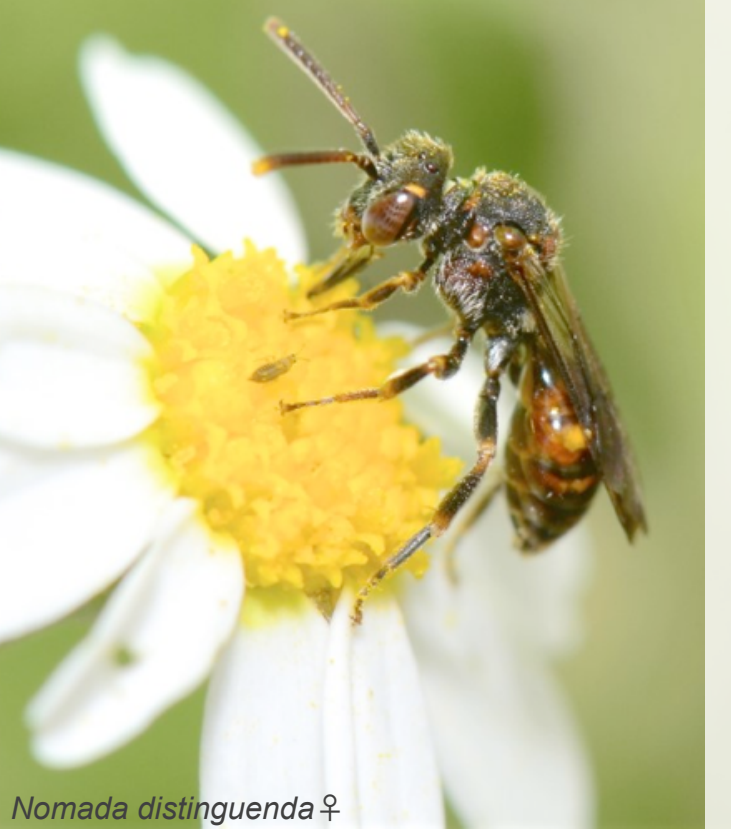

Nomada distinguenda우

\begin{tabular}{|l|}
\hline \multicolumn{1}{|c|}{ COMITÉ } \\
ÉDITORIAL \\
Editeur en chef \\
Benoît Geslin \\
Comité de relecture \\
Matthieu Aubert \\
Éric Dufrêne \\
David Genoud \\
Benoît Geslin \\
Tanguy Jean \\
Gérard Le Goff \\
Violette Le Féon \\
Léa Lemaire \\
Gilles Mahé \\
Denis Michez \\
Géraud de Prémorel \\
Nicolas J.Vereecken \\
\hline
\end{tabular}

\section{SOUMISSION D'ARTICLES}

\section{De l'importance des sciences naturalistes}

"If we do not know where bee species live, and how abundant they are, it is almost impossible to measure decline and generate prioritised and meaningful conservation strategies. ॥ Brown \& Paxton 2009!.

Pour beaucoup d'entre nous, cette phrase publiée dans la revue scientifique Apidologie il y a maintenant presque 10 ans raisonne comme un mantra, une profession de foi et elle est plus que jamais d'actualité. II est en effet tout à fait frappant de réaliser à quel point nous manquons encore de connaissances de base sur les abeilles sauvages malgré toutes les études, publications et autres analyses qui paraissent chaque année. Pour une partie non négligeable d'entre elles, nous ignorons encore tout simplement leur répartition, leur écologie et la dynamique de leur population. Dans ce cadre, le développement des connaissances naturalistes sur les Apoidés sauvages - une des aspirations de l'Observatoire des Abeilles - reste un objectif majeur qui doit tendre à se développer dans les années futures.

e nouveau numéro d'Osmia est une illustration parfaite de l'importance des sciences naturalistes pour la progression des connaissances sur les abeilles. En effet, ce ne sont pas moins de 3 espèces nouvelles pour la faune de France métropolitaine qui sont présentées dans ce septième numéro. Tout d'abord, E. Gabiot et E. Dufrêne font pour la première fois mention de Nomada numida dans l'hexagone. Une autre espèce du genre, Nomada rubricoxa, y a également été découverte récemment dans le cadre d'une étude menée dans le Parc National des Calanques (Geslin et al.). Enfin, Ghisbain et al. étoffent le genre Dasypoda en y ajoutant une neuvième espèce à savoir D. morawitzi. Ce numéro d'Osmia s'attache aussi à accroître les connaissances sur les communautés d'abeilles sauvages dans les milieux anthropisés (Paris, Ropars et al.) ou semi-naturels (Parc National des Calanques, Geslin et al.). Enfin, les aires de répartition nationale telles que connues à ce jour d'un de nos bourdons les plus majestueux, Bombus argillaceus, et d'une espèce exotique envahissante, Megachile sculptularis, font l'objet d'une présentation détaillée, respectivement par B. Frin ainsi que par V. Le Féon et al. Nous inaugurons également une nouvelle rubrique dans la partie Forum dans ce numéro puisque T.Jean présente ici ses conseils de lecture pour tous les passionnés de taxonomie et d'écologie des abeilles sauvages. Pour finir, vous y retrouverez une magnifique planche photo réalisée par $G$ de Premorel.

Nous espérons que vous apprécierez ce numéro autant que nous avons eu plaisir à le rédiger .... et que vivent les sciences naturalistes !

Benoît GESLIN, Editeur d'Osmia, pour le comité de rédaction. 


\section{Sommaire}

\section{Articles}

Première mention de Nomada numida LEPELETIER I84I (Apoidea - Apidae - Nomadini) pour la France continentale

Par E. GABIOT, E. DUFRÊNE.

Dasypoda morawitzi RADCHENKO 2016 (Apoidea - Melittidae - Dasypodaini), une espèce nouvelle pour la faune de France

Par G. GHISBAIN, V. G. RADCHENKO et D. MICHEZ ........................................ 10

La diversité des abeilles parisiennes

Par L. ROPARS, I. DAJOZ et B. GESLIN.

Compte rendu des captures réalisées lors de la formation Européenne à la détermination des abeilles (COST Super-B Project) dans le Parc National des Calanques

Par B. GESLIN et al.

Présence de Bombus argillaceus Scopoli 1763 (Apoidae - Apidae - Bombini) dans les Hautes-Alpes en France

Par B. FRIN

Écologie et distribution de l'abeille originaire d'Asie Megachile sculpturalis SMITH I853 (ApoideaMegachilidae - Megachilini) : un état des connaissances dix ans après sa première observation en Europe par V. LE FÉON et B. GESLIN. 31

Forum

Quelques notes de lecture ParT.JEAN. .40

Galerie photo

Oligolectisme chez les abeilles sauvages Par G. DE PRÉMOREL... 45 


\title{
Première mention de Nomada numida Lepeletier I84 I (Apoidea - Apidae - Nomadini) pour la France continentale
}

\author{
par Eric GABIOT*, Eric DUFRÊNE**
}

Résumé. La découverte de Nomada numida Lep. 1841 en France dans le Var au printemps 2017 est présentée. Les connaissances sur la répartition des deux sous-espèces ( $N$. numida ssp. numida Lep. 1841 N. numida ssp. manni Morawitz 1871) sont ensuite synthétisées. Une mise au point sur la répartition de Nomada mauritanica Lep. 1841 et de ses deux sous-espèces est donnée en complément.

Abstract. We present here the discovery of Nomada numida Lep. 1841 in France (Var) during the spring of 2017. The knowledge relative to the distribution of the two subspecies (N. numida ssp. numida Lep. 1841 and N. numida ssp. manni Morawitz 1871) is then synthesized. In addition, an update of the distribution of $N$. mauritanica Lep. 1841 and its two subspecies is given.

Mots-clés : Nomada numida, Nomada mauritanica, France, Var.

Keywords : Nomada numida, Nomada mauritanica, France, Var.

\section{Introduction}

Les Nomada sont dépendantes d'autres espèces d'abeilles sauvages pour réaliser leur cycle de vie. En effet, les femelles ne récoltent pas de pollen et pondent leurs oufs dans le nid d'autres espèces après que ces dernières aient accumulé les réserves nécessaires pour approvisionner la larve. Ce comportement est qualifié de cleptoparasite et les Nomada sont appelées " abeilles coucous " par analogie avec le comportement de l'oiseau.

Avec 101 espèces en France, le genre Nomada est le genre cleptoparasite le plus diversifié et le second genre en nombre d'espèces, après les Andrènes, et ce quel que soit le mode de vie considéré (RASMONT et al. 2017). Trois nouvelles espèces ont été découvertes depuis la mise à jour de DUFRÊNE et al. (2014). Les spécimens de deux d'entre elles, Nomada erythrocephala Morawitz

\footnotetext{
* Société des Sciences Naturelles et d'Archéologie de Toulon et du Var (https://www.ssnatv.fr/), 2 allée Amiral Courbet, 83000 Toulon - gabiot.eric@gmail.com

** Observatoire des Abeilles (https://oabeilles.net/), 9 allées des Pins, Malassis, 91470 Forges-les-Bains - eric.dfn@wanadoo.fr
}

1870 et Nomada trapeziformis Schmiedeknecht 1882 ont été retrouvés dans des collections et correspondent à des données anciennes (Jan Smit com. pers.). La troisième espèce, Nomada rubricoxa Schwarz 1977, dont la découverte en 2016 est évoquée dans un autre article du présent numéro d'OSMIA (GESLIN et al. 2018).

\section{Observations sur le terrain}

Au cours d'une sortie de la Société des Sciences Naturelles et d'Archéologie de Toulon et du Var (SSNATV), le 5 avril 2017, une femelle d'abeille du genre Nomada a été capturée au filet alors qu'elle volait à proximité de cistes cotonneux (Cistus albidus) en fleurs. Cette sortie, dont le but était d'évaluer la diversité entomologique, botanique, mycologique et géologique du site, s'est déroulée sur la commune d'Évenos dans le Var, sur la piste faisant le tour du Fort du Pipaudon (figure 1). Le sentier emprunté au cours de la sortie traverse successivement des sols sableux d'origine calcaire et des sables grésifiés, restes issus d'une ancienne carrière de marbre et de sable. Ces substrats meubles sont propices aux creusements de nids pour de nombreuses espèces d'abeilles sauvages. Début 


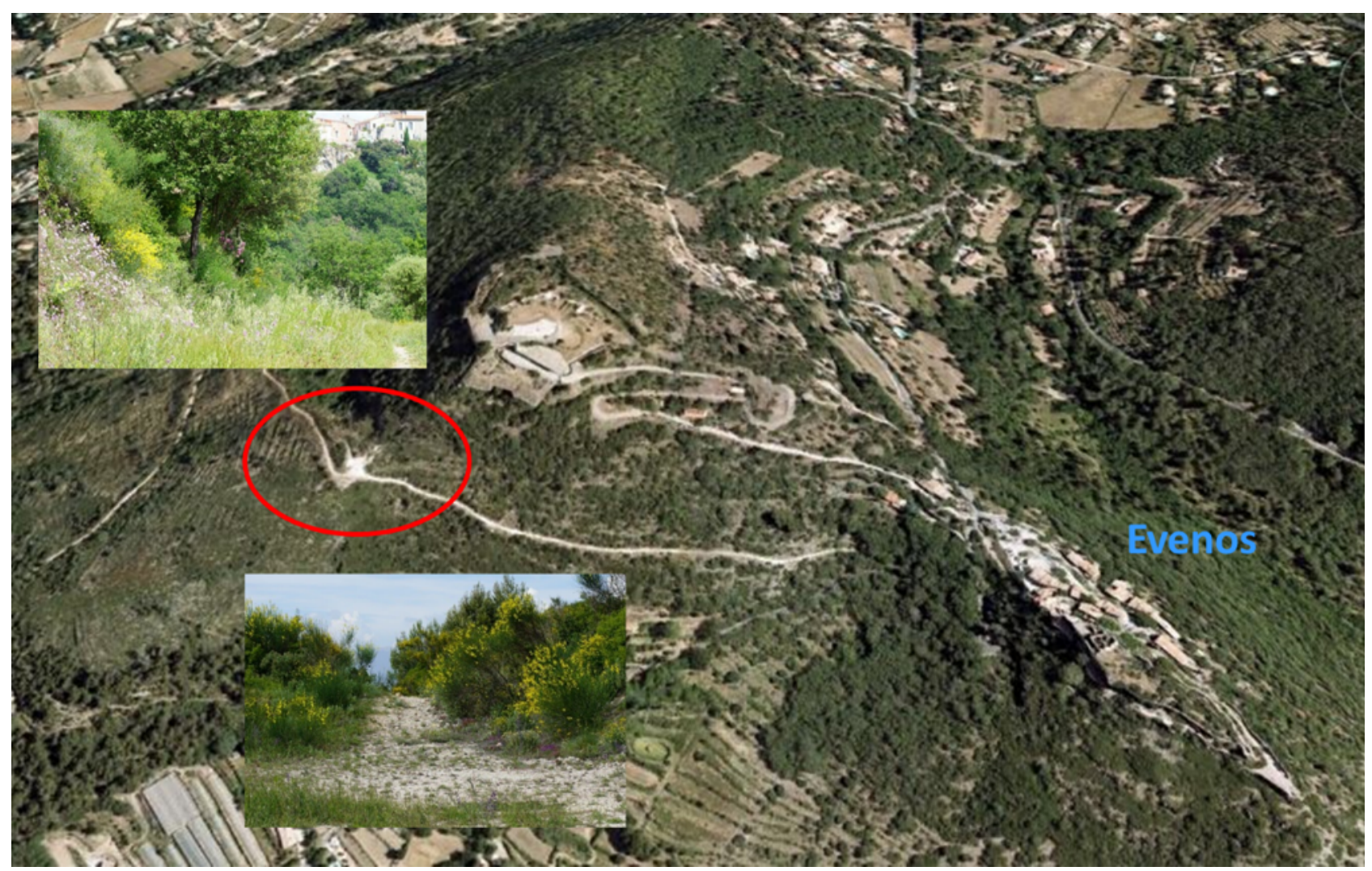

Figure I : localisation de la capture avec deux photos des milieux.

avril, la floraison était abondante comme c'est fréquent en climat méditerranéen, avec en particulier de nombreux pieds de cistes, chardons, bourraches, thyms, asphodèles, vipérines et genêts.

De nombreuses abeilles sauvages ont été capturées ce jour-là. Plusieurs individus d'Andrena morio (mâles et femelles) ont pu ainsi être observés sur les fleurs de cistes cotonneux.

C'est à cette occasion et faisant suite à la capture d'une femelle d'Andrena morio qu'une Nomada a été capturée. Après examen à la binoculaire, celle-ci s'est avérée être $N$. numida numida.

\section{Statut systématique de Nomada numida et de Nomada mauritanica et de leurs sous- espèces}

Jusqu'à la mise au point de ScHWARZ \& GUSENLEITNER (2003), il régnait une certaine confusion dans la nomenclature des deux espèces N. numida Lep. 1841 et $N$. mauritanica Lep. 1841 et de leurs sous-espèces respectives à savoir N. numida ssp. numida Lep. 1841, N. numida ssp. manni Mor. 1871, N. mauritanica ssp. mauritanica Lep. 1841 et $N$. mauritanica ssp. chrysopyga Mor. 1871.
Précédemment, N. chrysopyga Mor. 1871 était considérée comme une espèce valide et $N$. manni était considérée comme une sous-espèce de $N$. mauritanica. Les deux espèces et leurs sousespèces respectives appartiennent à deux "groupes" différents (définis au sens d'ALEXANDER \& SCHWARZ, 1994). A noter qu'une inversion s'est produite dans la publication de DUFRÊNE et al. (2014; tab. II p. 1490) : $N$. numida appartient en fait au « groupe bifasciata " et $N$. mauritanica au "groupe ruficornis ". Les critères d'identification du "groupe bifasciata" sont bien établis, pour les mâles comme pour les femelles, évitant ainsi toute ambiguïté.

Les deux espèces $N$. numida et $N$. mauritanica et leurs deux sous-espèces respectives sont très variables en taille et en coloration ce qui a entretenu la confusion. Les femelles de $N$. numida se distinguent aisément par la présence de deux (rarement trois) petites épines accolées et fortement coudées à l'apex du tibia postérieur, comme chez toutes les espèces du " groupe bifasciata ". Les femelles de $N$. mauritanica présentent deux ou trois petites épines droites et bien séparées à l'apex du tibia postérieur. 
Les deux sous-espèces de $N$. numida sont plus difficiles à séparer en raison de leur grande variabilité et des critères exclusivement basés sur des différences de coloration. La sous-espèce nominale présente un abdomen orange à rouille, sans jaune (parfois orange délavé), souvent sans noir avec quelques exceptions toutefois. La sousespèce manni présente une bande jaune vif transversale, au centre des tergites 2 à 5 , parfois aussi du jaune sur le tergite 1. La base des tergites 3 et 4, parfois aussi le 2 et le 5 , présente une bande noire bien marquée. La coloration de la tête et du thorax est trop variable pour être discriminante.

\section{Le spécimen capturé dans le Var}

La femelle capturée dans le Var présente un habitus (figure 2) très proche de N. mephisto Schmied., 1882 [= N. numida numida Lep. 1841] tel que figuré dans ScHWARZ \& GUSENLEITNER (2015 ; fig. 34 à 36, p. 1042). Si l'abdomen est tout à fait similaire, la tête et le thorax présentent un peu de coloration jaune : les taches $\mathrm{du}$ scutellum et post-scutellum sont jaunes de même que les calli, les bandes colorées longeant la partie interne de l'œil sont majoritairement jaunes. Avec à peine $10 \mathrm{~mm}$ de longueur, l'individu capturé est de petite taille pour l'espèce. Toutefois des spécimens de cette taille se rencontrent occasionnellement en Espagne (Maximilian Schwarz, com. pers.).

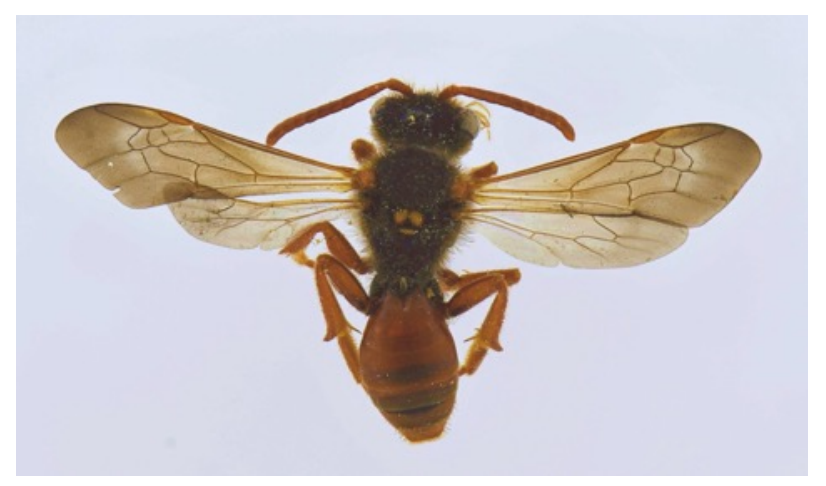

Figure 2 : photo du spécimen - habitus

\section{Distribution géographique}

La confusion passée dans la nomenclature (nombreuses synonymies) et la variabilité en taille et coloration des deux espèces ( $N$. numida et $N$. mauritanica) rendent difficile l'établissement d'une répartition géographique précise et fiable.
Les sites internet traitant de ce sujet ne sont pas encore à jour. Ainsi $N$. chrysopyga Mor. 1871 est toujours considérée comme une espèce valide sur les sites ITIS et GBIF. Sur Westpalbees (KuHLMANN et al. 2017), seules les deux espèces valides sont considérées mais il s'y trouve des confusions dans la synonymie. Par conséquent, les répartitions indiquées sur ces sites sont sujettes à caution et ne peuvent être considérées directement.

Nous avons donc repris les publications originales ainsi que les spécimens des collections $\mathrm{du}$ MNHN (Paris) en utilisant la synonymie donnée par SCHWARZ \& GUSENLEITNER (2003). Ces données ont été complétées par les spécimens de la collection de l'un d'entre nous (E.D.) et par des informations communiquées par M. Schwarz. Dans l'état actuel des connaissances, on peut considérer que les distributions suivantes sont avérées même si probablement incomplètes :

$N$. numida ssp. numida Lep. est connue des trois pays du Maghreb, d'Espagne y compris les Baléares, de Croatie (côte dalmate) et de Russie méridionale (sud de Volvograd).

$N$. numida ssp. manni Mor. est bien présente sur les îles méditerranéennes (Corse, Sardaigne, Cyclades-Tinos, Crète) ainsi qu'en Grèce (Epire), Croatie (côte dalmate) et Italie (Calabre).

N. mauritanica ssp. mauritanica Lep. semble limitée à l'Afrique du Nord (Algérie, Tunisie et Egypte).

N. mauritanica ssp. chrysopyga Mor. semble absente d'Afrique du Nord. Elle présente une large répartition sur les rives nord et orientale de la Méditerranée (Espagne, France continentale, Italie dont Sicile, Grèce, Crète, Syrie) mais se rencontre aussi plus au nord en Hongrie et en Autriche.

En ce qui concerne la France, il est bien établi qu'aucune des deux sous-espèces de $N$. numida n'avait été mentionnée de France continentale. Pour la Corse, seule la sous-espèce $N$. numida ssp. manni Mor. est présente et assez fréquente.

Concernant $N$. mauritanica Lep., seule la sousespèce $N$. mauritanica ssp. chrysopyga Mor. est présente en France continentale où elle est rare. Elle se rencontre principalement en zone 
méditerranéenne mais remonte jusqu'à Lyon. Sa présence en Corse n'est pas avérée.

\section{Discussion et conclusion}

La découverte remarquable de $N$. numida ssp. numida Lep. en France continentale pose, comme pour d'autres espèces du genre découvertes récemment, la question de l'ancienneté de sa présence sur notre territoire. Compte tenu de la grande distance relative qui la sépare de ses bastions espagnols, il est probable que cette découverte relève plus de la méconnaissance que nous avons de notre faune des abeilles plutôt que d'une forte dynamique des populations espagnoles, même si cette dernière hypothèse ne peut être complètement écartée. La très forte augmentation du nombre d'espèces de Nomada (DUfRÊNE et al. 2014) et plus généralement d'abeilles (RASMONT et al. 1995 ; RASMONT et al. 2017) en France depuis 1995 ne peut trouver son explication que dans la dynamique d'inventaires initiée au début des années 2000 après presque un siècle de " basses eaux ". Si les changements environnementaux, et en particulier le climat, jouent un rôle dans cette très forte augmentation du nombre d'espèces, ce rôle est certainement modeste et ne peut pas actuellement être mis en évidence.

Il est notable qu'en dépit de la présence de $N$. numida ssp. manni Mor. en Corse, en Italie (Sardaigne et Calabre) ainsi qu'en Croatie (côte dalmate), ce soit l'autre sous-espèce $N$. numida ssp. numida Lep., connue d'Espagne et de Croatie (côte dalmate), qui soit présente dans le Var.

\section{Remerciements}

Un grand merci à Maximilian Schwarz pour les informations communiquées sur ces deux espèces de Nomada et leurs sous-espèces respectives. Nous remercions Agnièle Touret-Alby, conservatrice de la collection d'Hyménoptères du Muséum de Paris, pour son accueil et son aide au cours de nos recherches.

\section{Références bibliographiques}

AleXANDer BA \& Schwarz M, 1994. A catalog of the species of Nomada (Hymenoptera Apoidea) of the world. The University of Kansas Science Bulletin 55 (7) : 239-268.
Dufrêne E, Schwarz M, SMit J, 2014. Le genre Nomada Scopoli en France continentale et en Corse : citation de 15 espèces nouvelles pour la faune de France et mise à jour de la liste taxonomique des espèces (Hymenoptera: Apoidea, Anthophila). Linzer biologische Beitrage 46 (2) : 1479-1490.

Geslin B, Vereecken NJ, Roberts R, Murat Aytekin A, Genoud D, Aubert M, Burdon RCF, Ruiz C, FisogNi A, FlaCher F, Grabowski M, JaCqUemin F, KhVir V, KirkitAdZe G, KLUMPERS SGT, LEVy K, MAHER S, MARKOV Z, GERRARD A, ROQUER L, Ropars L, Schurr L, VARnava A, Michez D, 2018. Compte rendu des captures réalisées lors de la formation européenne à la détermination des abeilles (COST Super-B Project) dans le Parc National des Calanques. Osmia 7 : 20-25.

Kuhlmann M, Ascher JS, DAThe HH, Eimer AW, Hartmann $P$, Michel $D$, Müller $A$, Patiny S, Pauly a, Praz C, Rasmont P, Risch S, Scheuchl E, Schwarz M, Terzo M, Williams PH, AMiet F, BALdock D, Berg Ø, Bogusch P, Calabuig I, Cederberg B, Gogala A, Gusenleitner F, Josan Z, MADSEN HB, NilsSON A, ØDEGAARD F, ORTIZSanchez J, Paukkunen J, PaWlikowski T, QUARANTA M, ROBERTS SPM, SAROPATAKI M, SCHWENNINGER HR, SMIT J, SöDERMAN G, TOMOzEI B (2017/09/12). Checklist of the Western Palaearctic Bees (Hymenoptera : Apoidea : Anthophila). http:// westpalbees.myspecies.info.

RASMONT P, EBMER P.A, BANASZAK J.G \& VAN DER ZANDEN G, 1995. Hymenoptera Apoidea Gallica. Liste taxonomique des abeilles de France, de Suisse et du Grand-Duché de Luxembourg. Bulletin de la Société entomologique de France 100 (hors-série) : 1-98.

Rasmont P, Genoud D, Gadoum S, Aubert M, Dufrêne E, LE GofF G, MAHÉ G, Michez D \& Pauly A, 2017. Hymenoptera Apoidea Gallica : liste des abeilles sauvages de Belgique, France, Luxembourg et Suisse. Atlas Hymenoptera, Université de Mons, Mons, Belgium. http://www.atlashymenoptera.net/

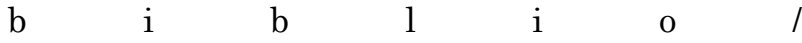
414_Rasmont_et_al_2017_Hymenoptera_Apoidea _Gallica_2017_02_16.pdf

SChWARZ M \& Gusenleitner F, 2003. Ergebnis der Untersuchung einiger von SPINOLA beschriebener Apiden mit Bemerkungen und Ergänzungen. (Hymenoptera Apidae). Entomofauna 24: 237-280. 
SChWARZ M \& GUSENLEITNER F, 2015. Über den Verbleib der von SCHMIEDEKNECHT 1882 beschriebenen Nomada-Arten und Festlegung von Lectotypen der aufgefundenen Arten. Mit zusätzlichen Ergänzungen und Beschreibungen $\mathrm{zu}$ einigen Arten (Hymenoptera, Apidae, Nomadinae). Linzer biologische Beitrage 47 (1) : 1003-1044.

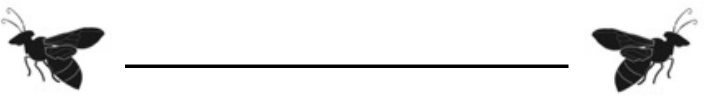




\section{Dasypoda morawitzi RADCHENKO 2016 (Apoidea - Melittidae - Dasypodaini), une espèce nouvelle pour la faune de France}

par Guillaume GHISBAIN*, Vladimir G. RADCHENKO** et Denis MICHEZ*

Résumé. Dasypoda morawitzi RADCHEnKo 2016, Melittidae cryptique longtemps confondue avec l'espèce à large distribution Dasypoda hirtipes, est pour la première fois recensée en France.

Abstract. Dasypoda morawitzi RADChEnKo 2016, a cryptic Melittidae mistakenly identified as the large-distribution species Dasypoda hirtipes for a long time, is recorded in France for the first time.

Mots-clés : Dasypoda morawitzi ; Melittidae ; France

$\mathrm{Au}$ sein des Melittidae, l'une des plus petites familles d'apoïdes en termes de nombre d'espèces (MICHENER 2000), se trouve le genre paléarctique Dasypoda LATREILle 1802. Les scopae notablement développées des femelles et la nervation de leurs ailes antérieures, à deux cellules submarginales, distinguent aisément les Dasypoda des autres genres inclus dans la famille des Melittidae. Le recensement le plus récent de la faune d'abeilles de France comptabilise actuellement, pour le genre Dasypoda, 8 espèces (RASMONT et al. 2017b) sur les 28 espèces de la révision ouest-paléarctique de Michez et al. (2004). L'espèce Dasypoda hirtipes (FABRICIUS 1793), largement répandue au sein du Paléarctique attira cependant l'attention de RADCHENKO (2016) qui observa des différences morphologiques notables entre des individus de collection identifiés comme Dasypoda hirtipes. Sur la base de plusieurs caractères morphologiques incluant la pilosité des genitalia des mâles et la morphologie des galéas des deux sexes, RADCHENKo (2016) décrit la nouvelle espèce Dasypoda morawitzi, $29^{\mathrm{ème}}$ espèce de Dasypoda dans la région ouestpaléarctique (RASMONT et al. 2017a) (figure 1).

\footnotetext{
* Laboratoire de Zoologie, Université de Mons, bâtiment

Pentagone, 7000 Mons, Belgique -

guillaume.ghisbain@student.umons.ac.be et denis.michez@umons.ac.be

** Institute for Evolutionary Ecology of the National Academy of Sciences of Ukraine, acad. Lebedev 37, Kyiv 03 I43, Ukraine rvg@nas.gov.ua
}

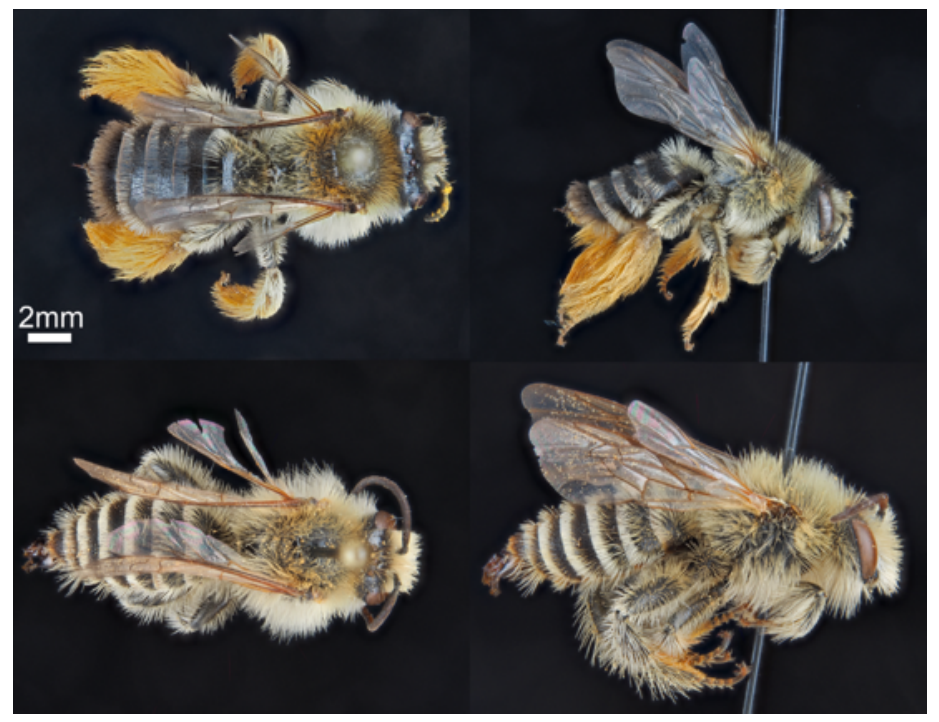

Figure I. Habitus en face dorsale et latérale de Dasypoda morawitzi, femelle en haut, mâle en bas (photos P. Rasmont).

En plus de sa présence en Russie, Ukraine et pays adjacents (RADCHENKO 2016), une publication récente de SCHMID-EGGER et DUBITZKY (2017) recense l'espèce en Europe centrale : l'Allemagne, l'Autriche, ainsi que la Bulgarie sont ajoutées à la distribution de D. morawitzi. L'espèce a aussi été recensée dans les îles grecques orientales (RASMONT et al. 2017a). Nous avons recensé l'espèce pour la première fois en France grâce à des collectes réalisée les 17, 19 et 20 juillet 2017 dans les communes de Peyrins et de Génissieux (département de la Drôme (26)), où mâles et 
femelles furent capturés sur des Asteraceae (habitus illustrés en figure 1). Une étude détaillée des collections du laboratoire de Zoologie de l'Université de Mons nous a également permis d'identifier d'autres spécimens dont les informations relatives à la capture sont détaillées ci-dessous. En France, la distribution de cette espèce semble se limiter à la zone méditerranéenne (figure 2).

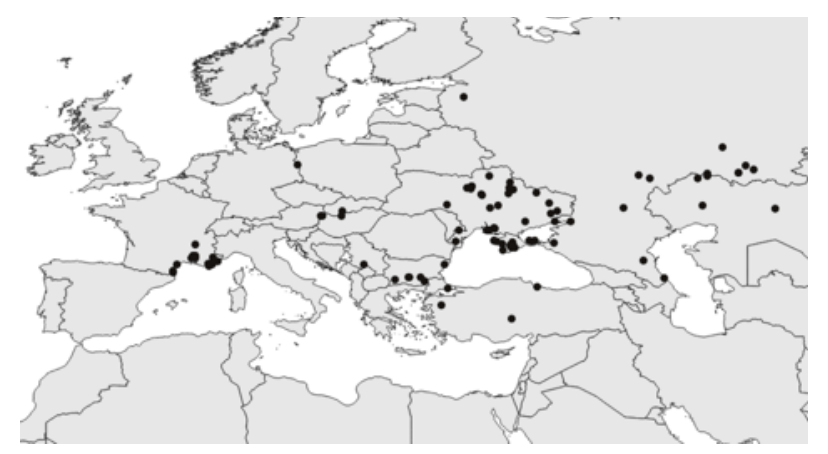

Figure 2. Distribution connue de Dasypoda morawitzi, reprenant les données de Radchenko (2016), Schmid-Egger et Dubitzky (2017) et de la présente étude.

\section{Données additionnelles de distribution et choix floraux}

Sur les 256 spécimens identifiés comme $D$. hirtipes dans les collections de l'Université de Mons, 51 femelles et 27 mâles ont été révisés comme $D$. morawitzi. Les informations de récolte tirées des étiquettes de ces spécimens sont les suivantes : France, Alpes-Maritimes (06), Antibes, mâle récolté le 26 juillet 1962 par J. Mathot ; France, Alpes-de-Haute-Provence (04), mâles récoltés le 18 juillet 1965 entre Annot et Fugeret sur Jasione montana ; France, Alpesde-Haute-Provence, mâle collecté à Annot le 27 juillet 1966 ; France, Var (83), Vidauban (Miquelette), femelles récoltées sur Cichorium intybus le 30 août 1996 par S. Patiny ; France, Var, Plan de la Tour, femelles récoltées sur Crepis vesicaria le 29 août 1996 par S. Patiny; France, Var, Bormes-les-Mimosas (L'Estagnol), femelle récoltée le $1^{\mathrm{er}}$ juillet 1988 par P. Rasmont sur Cichorieae ; France, Var, Gonfaron, femelle capturée le 23 août 1977 par Rasmont P. et Wéry ; France, Var, Saint-Tropez, femelle récoltée sur C. intybus le 27 août 1996 par S. Patiny ; France, Vaucluse (84), Ile d'Oiselay, femelle récoltée par piège à coupelle bleue le 21 août 2009 par Guilbaud et Paganin ; France, Vaucluse, Courthézon, femelle récoltée par piège à coupelle bleue le 21 août 2009 par Guilbaud et
Paganin ; France, Vaucluse, Lagnes, femelle capturée le 15 juillet 1980 ; France, Vaucluse, Carpentras, mâle collecté sur Malva le 22 juillet 1981 ; France, Gard (30), Aramon, femelle capturée le $1^{\text {er }}$ août 1979 ; France, Hérault (34), Portiragnes-Plage, femelles capturées le 18 juillet 2003 sur Cichorioideae par D. Michez; France, Hérault, Portiragnes-Plage, mâle capturé le 18 juillet 2003 sur Cichorioidae par D. Michez ; France, Pyrénées-Orientales (66), Argelès-plage, femelle capturée le 8 juillet 1965 par J.v.D. Vecht sur Cichorium ; France, Pyrénées-Orientales, Canet, mâle récolté le 3 juillet 1956 ; Serbie, Raska, femelle récoltée le 4 août 1972 par Wahis ; Slovaquie, Levice, femelle capturée le $1^{\text {er }}$ août 1961 par Kocourek ; Slovaquie, Cenkov, mâle capturé le $1^{\text {er }}$ août 1999 par M. Snizek ; Bulgarie, Melnik, mâle capturé le 13 août 1993 par M. Halada ; Bulgarie, Plovdiv, mâles capturés le $1^{\text {er }}$ juillet 1997 par Zaykov ; Bulgarie, Trakia, mâle récolté le 20 juillet 1996 par Zaykov ; Bulgarie, Razlog, mâle capturé le 13 août 1970 par Fraser et Jenkins ; Bulgarie, Trakia, Voïvodine, mâle capturé le 5 août 1997 par Zaykov ; Bulgarie, Svilengrad, mâle capturé le 5 juillet 2000 par M. Snizek ; Bulgarie, Rodopi, Galabovo, mâle capturé le 25 juillet 1997 par Zaykov ; Bulgarie, Zlaté Piesky, mâle récolté en juin 1986 par J.a.M. Halada ; Turquie, Istanbul, Selimpasa, femelles récoltées le 5 juillet 1966 ; Turquie, Samsun, Dikbiyik, mâles capturés le 11 juillet 1967 ; Turquie, Balikesir, Erdek, mâle récolté le 16 juillet 1967 ; Turquie, Istanbul, Selimpasa, mâle collecté le 5 juillet 1966.

Des sessions de collecte de 2017 ont permis de répertorier l'espèce dans deux stations de la Drôme en France : Peyrins, La Savasse (4505’53”N 504'31"E, $275 \mathrm{~m})$, 19.VII.2017 sur Asteraceae (dét. par G. Ghisbain) et Génissieux, Montée St-Christophe $\left(45^{\circ} 05^{\prime} 19^{\prime \prime} \mathrm{N} 5^{\circ} 04^{\prime} 17\right.$ ' E, 260m), 17.VII.2017 sur Asteraceae (dét. par G. Ghisbain).

Les données issues des collections l'Université de Mons ont révélé la présence de l'espèce en Serbie, en Slovaquie et ont confirmé sa présence en France. Ces informations corroborent également une nette association entre les femelles de cette espèce et la famille des Asteraceae. Les mâles semblent quant à eux interagir avec un panel floral plus important, englobant également des Malvaceae et Campanulaceae. 

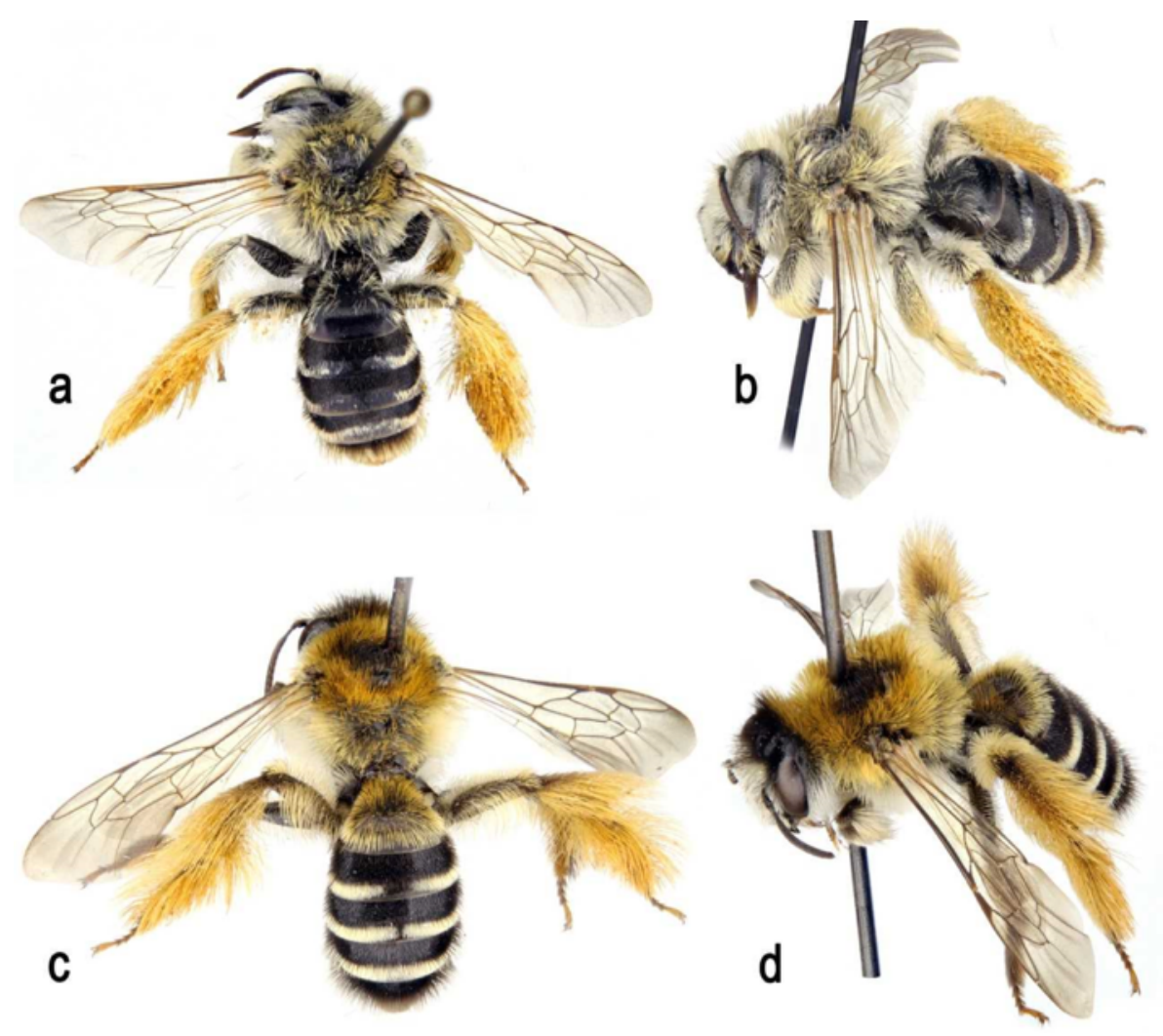

Figure 3. Comparaison des femelles de Dasypoda morawitzi (a, b) et Dasypoda hirtipes (c, d) (photos V. G. Radchenko).

Une révision des spécimens mal identifiés comme $D$. hirtipes dans les collections paléarctiques compléterait la distribution de $D$. morawitzi qui semble vraisemblablement se superposer partiellement à celle de son espèce sœur cryptique. L’identification est relativement aisée sur la base de caractères morphologiques typiques bien décrits par RADCHENKO (2016) (figures 3-4). Notons comme caractère diagnostique net la pilosité des genitalia des mâles, illustrés dans la figure 5 .

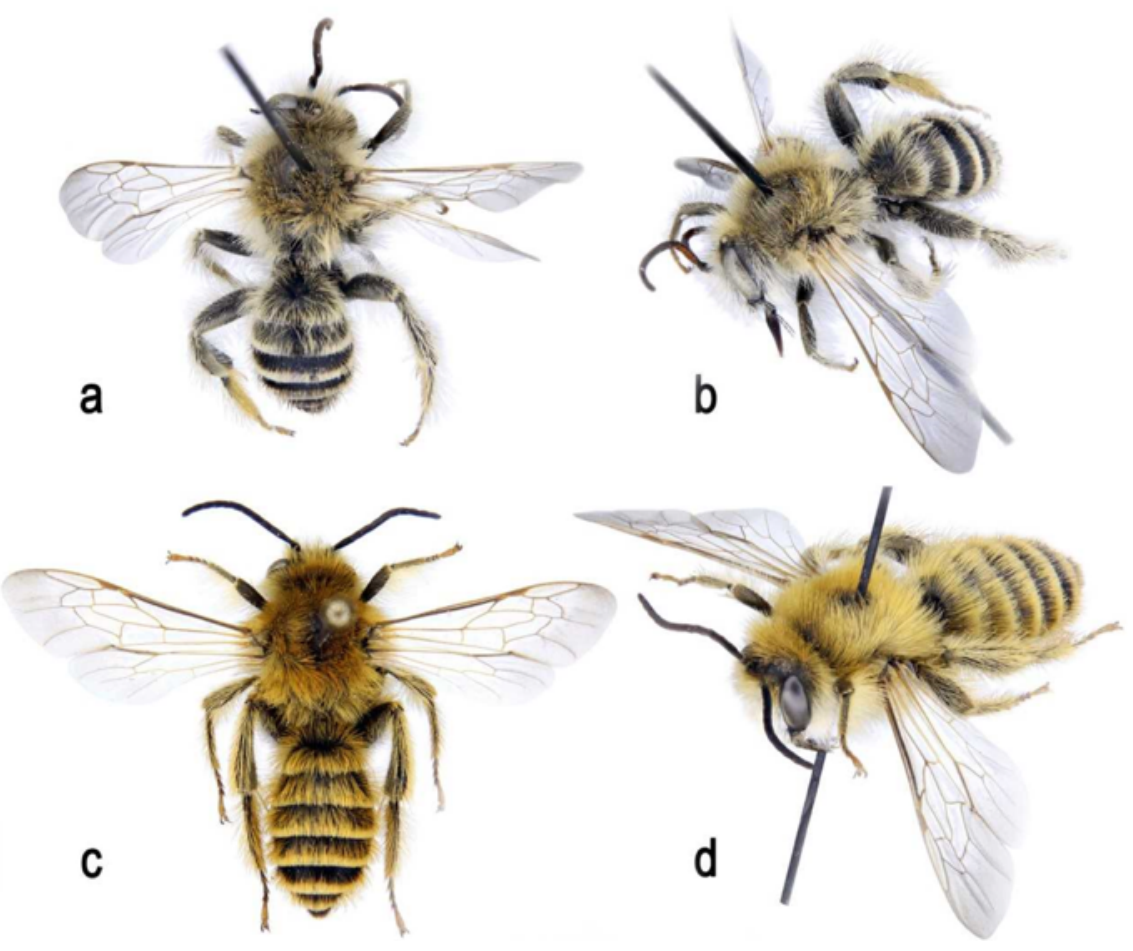

Figure 4. Comparaison des mâles de Dasypoda morawitzi (a, b) et Dasypoda hirtipes (c, d) (photos V. G. Radchenko). 

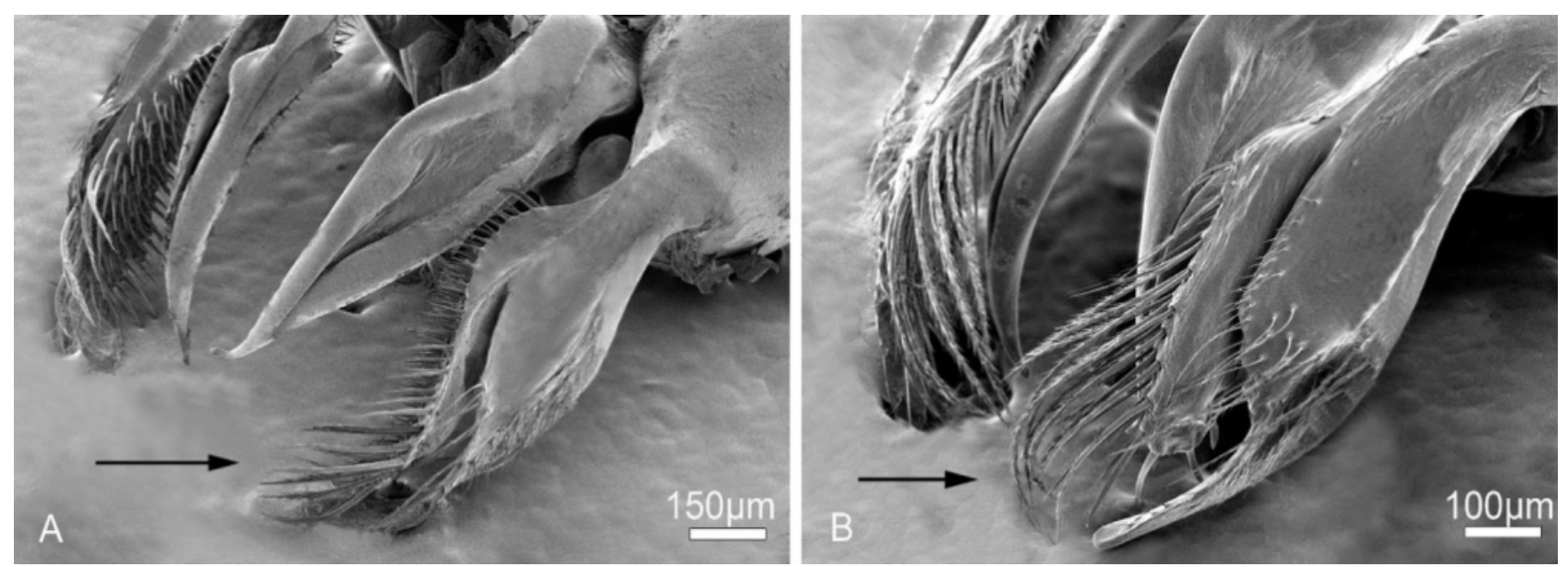

Figure 5. Détail par microscopie électronique des genitalia des mâles de Dasypoda morawitzi (A) et Dasypoda hirtipes (B). Les flèches indiquent la nette différence de pilosité au niveau des gonostyles des deux espèces (photos G. Ghisbain).

\section{Références bibliographiques}

MICHENER CD, 2000. The bees of the world. The Johns Hopkins University Press, Baltimore.

Michez D, Terzo M, RASmont P, 2004. Révision des espèces ouest-paléarctiques du genre Dasypoda Latreille 1802 (Hymenoptera, Apoidea, Melittidae). Linzer Biologische Beitrage 36(2): 847 - 900.

RADCHENKo VG, 2016. A new widespread European bee species of the genus Dasypoda Latreille (Hymenoptera, Apoidea). Zootaxa 4184: 491 - 504.

Rasmont P, Devalez J, Pauly A, Michez D \& RADChenko VG, 2017a. Addition to the checklist of IUCN European wild bees (Hymenoptera: Apoidea). Annales de la Société entomologique de France (N.S.) 53: 17 - 32.

Rasmont P, Genoud D, Gadoum S, Aubert M, Dufrêne E, Le Goff G, MAhÉ G, Michez D \& Pauly A, 2017b. Hymenoptera Apoidea Gallica: liste des abeilles sauvages de Belgique, France, Luxembourg et Suisse. Atlas Hymenoptera, Université de Mons, Mons, Belgium. http://www.atlashymenoptera.net (consulté le 28/02/2018).

SChmid-EgGer C \& Dubitzky A, 2017. Dasypoda morawitzi (Radchenko, 2016) neu für die Fauna von Mitteleuropa (Hymenoptera, Apoidea). Ampulex - Zeitschrift für aculeate Hymenopteren 9: 27 - 31. 


\title{
La diversité des abeilles parisiennes
}

\author{
par Lise ROPARS***, Isabelle DAJOZ** et Benoît GESLIN*
}

\begin{abstract}
Résumé. Malgré les perturbations dues à l'urbanisation grandissante, les villes abritent une biodiversité non négligeable. Comme beaucoup d'autres taxons, les abeilles sauvages peuvent être relativement abondantes dans les zones urbaines du fait de certaines conditions favorables par rapport aux milieux agricoles (absence de pesticides, îlot de chaleur urbain, floraison toute l'année). Dans cette étude, nous compilons nos travaux conjoints afin d'enrichir la liste des espèces d'abeilles parisiennes. Au total, le suivi de 4 années (2011 puis 2014, 2015, 2016) a permis de recenser 87 espèces d'abeilles à Paris intra-muros. L'ajout en 2016 de la méthode de capture avec des filets à papillon en plus de la méthode passive avec coupelles colorées a permis de capturer 11 espèces supplémentaires. L'assemblage, qui représente à ce jour 8,8 \% de la diversité spécifique des abeilles sauvages de France métropolitaine, est cependant dominé par les Halictidae et ne compte que quelques espèces cleptoparasites, témoignant d'une communauté appauvrie.
\end{abstract}

\begin{abstract}
Despite the forever increasing urbanization, cities often shelter a significant biodiversity. As many other taxa, wild bees may find refuge in urban areas because of relative favorable conditions compared to intensive agricultural environments. In this study, we compiled our joint work to extend the list of Parisian bees. Over 4 years of monitoring $(2011,2014,2015,2016)$, we recorded 87 bee species in intra-muros Paris. In 2016 we added net captures to our sampling procedures with colored pan traps, which provided 11 additional species. The assemblage now represents $8.8 \%$ of the French fauna of Apoïdae. However, this assemblage is mostly dominated by Halictidae and includes only few kleptoparasitic species highlighting an impoverished community.
\end{abstract}

Mots-clés : abeilles sauvages, Paris, écologie urbaine.

Keywords : wild bees, Paris, urban ecology.

\section{Introduction}

Les insectes pollinisateurs sont en fort déclin depuis une soixantaine d'années (PоTтs et al. 2016). Plusieurs facteurs sont à l'origine de cette régression notamment la destruction des habitats naturels, l'utilisation de pesticides, la perte de ressources florales ou encore l'introduction d'espèces envahissantes (GOULSON et al. 2015). En dépit de l'effet négatif de l'urbanisation sur les communautés de pollinisateurs (DEGUINES et al. 2016), plusieurs études ont montré que les villes pouvaient abriter un certain nombre d'espèces d'abeilles (BALDOCK et al. 2015; HALL et al. 2016). Par exemple, 291 espèces ont été recensées sur le Grand Lyon sur 2 ans d'échantillonnage (FORTEL

\footnotetext{
*Aix Marseille Univ, Univ Avignon, CNRS, IRD, IMBE, Marseille, France

** Institut d'Ecologie et des Sciences de l'Environnement de Paris (iEES Paris UMR CNRS 7618), Equipe Ecologie et Evolution des réseaux d'interactions, Université Paris Diderot, CNRS-UPMC.
}

et al. 2014). Les habitats favorables pour les abeilles sauvages doivent à la fois proposer une offre de nourriture suffisante ainsi que des sites de nidifications variés (WESTRICH 1996). Contrairement à une grande partie des espaces agricoles actuels, les espaces verts présents en zones urbaines et péri-urbaines offrent une grande diversité d'espèces de plantes à fleurs, sont moins traités avec des pesticides et bénéficient de températures en moyennes plus douces dues à l'îlot de chaleur urbain (BALDOCK et al. 2015).

En 2011, GESLIN et al. (2016) ont dénombré 44 espèces d'abeilles dans les parcs urbains de Paris. En 2014 et 2015, nos travaux conjoints ont permis d'étendre cette liste à 67 espèces (ROPARS et al. 2017). Nous présentons ici une mise à jour des espèces présentes à Paris avec l'ajout des spécimens capturés en 2016. 


\section{Matériel et Méthodes}

\section{Sites d'études}

Toutes les captures d'abeilles ont été conduites dans la ville de Paris, qui couvre près de $105 \mathrm{~km}^{2}$. Cette ville possède plus de 2.2 millions d'habitants (INSEE 2014) et dispose seulement de 3000 ha d'espaces verts ce qui équivaut à $14,5 \mathrm{~m}^{2}$ ou $5,8 \mathrm{~m}^{2}$ de surface végétalisée par habitant si on prend en compte ou non la surface des deux bois urbains (Vincennes et Boulogne) (APUR 2004). Cela est faible par rapport aux capitales voisines avec $36 \mathrm{~m}^{2}$ d'espaces verts par habitant à Amsterdam, $45 \mathrm{~m}^{2}$ à Londres, $59 \mathrm{~m}^{2}$ à Bruxelles ou encore $321 \mathrm{~m}^{2}$ à Rome (APUR 2004). Le climat parisien est océanique avec une température moyenne annuelle de $12,4^{\circ} \mathrm{C}$, un minimum de $4,9^{\circ} \mathrm{C}$ en janvier et un maximum de $20,5^{\circ} \mathrm{C}$ en juillet (http://www.infoclimat.fr).

\section{Suivi des abeilles sauvages}

Dans cette étude, nous compilons les données des espèces capturées en 2011, 2014, 2015 et 2016. Les sites sont localisés dans des squares, parcs urbains ou campus universitaires de Paris (figure 1). Pour chacun d'entre eux, les abeilles ont été capturées soit par méthode active soit par méthode passive soit en employant l'une et l'autre et systématiquement lorsque les conditions météorologiques étaient favorables (température $>12^{\circ} \mathrm{C}$, journée ensoleillée et faible vent). La méthode a consisté à capturer au filet pendant 15 minutes toutes les abeilles en train de butiner sur des patchs de végétation très fleuris $\left(3 \mathrm{~m}^{2}\right)$. La méthode passive, quant à elle, a consisté en l'emploi de pièges sous la forme de coupelles colorés (une blanche, une jaune, une bleue) laissés sur le terrain $24 \mathrm{~h}$ et préalablement peintes avec des peintures reflétant les ultra-violets auxquels sont sensibles les abeilles. Pour rendre effectif le piégeage, chaque coupelle doit être remplie d'eau (légèrement) savonneuse. Sur une station, les trois coupelles sont disposées linéairement et espacées d'1 m entre elles (WeStPHAL et al. 2008). Ces deux méthodes se complètent puisque celle dite passive sous-estime la présence et l'abondance des abeilles les plus grandes (Bombus, Colletes) tandis que la méthode active sous-estime la présence des abeilles les plus petites (Halictus, Lasioglossum) (WILSON et al. 2008 ; ROULSTON et al. 2007).
Le détail du protocole a varié d'une année à l'autre. En voici les détails :

- en 2011, GESLIN et al. (2016) ont capturé par méthode passive pendant 15 sessions d'échantillonnage de mi-avril à mi-juillet dans 3 grands parcs parisiens à savoir le Jardin des Plantes, le Jardin du Luxembourg et le Jardin de la Cité Internationale Universitaire de Paris ; - en 2014, Ropars et al. (2017) ont capturé par méthode passive pendant 10 sessions d'échantillonnage de mi-mai à mi-juillet dans 5 localités qui sont les campus des Universités Pierre et Marie Curie, Paris Descartes et Paris Diderot, de l'Institut de Physique du Globe de Paris et le Parc de Bercy (un essai expérimental a également été conduit à la Bibliothèque Nationale de France avec une seule pose de coupelles colorées - ayant fourni 4 abeilles) ;

- en 2015 , les 5 localités précédentes ont été rééchantillonnées par méthode passive avec l'ajout de 2 nouvelles localités (Jardin du Collège de France et Jardin de la Bibliothèque Nationale de France) pendant 13 sessions d'échantillonnage de mi-avril à mi-juillet.

- en 2016, les 7 localités précédentes ont été inventoriées pour la $3^{\mathrm{e}}$ année consécutive employant systématiquement les deux méthodes pendant 12 sessions d'échantillonnage de début avril à mi-juillet.

Les spécimens capturés dans les coupelles ont été conservés dans de l'alcool à $70^{\circ}$ jusqu'à leur préparation (rinçage, épinglage, séchage) dans le but de les déterminer à l'espèce. Ceux capturés au filet ont été tués en les plaçant dans des bocaux avec des granulés imbibés d'acétate d'éthyle. Ils ont ensuite été épinglés et déterminés suivant la nomenclature de KUHLMANN et al. (2013).

Enfin, à partir des données de la littérature, les informations sur les traits écologiques ont été renseignées pour chaque espèce. Les traits écologiques qui ont été considérés sont le type de nidification, la sociabilité, le caractère parasite et le lectisme (GENOUD 2017 ; Atlas Hymenoptera ; FlorAbeilles) ainsi que le statut de conservation tel que défini dans la liste rouge Européenne (NIETO et al. 2014). 


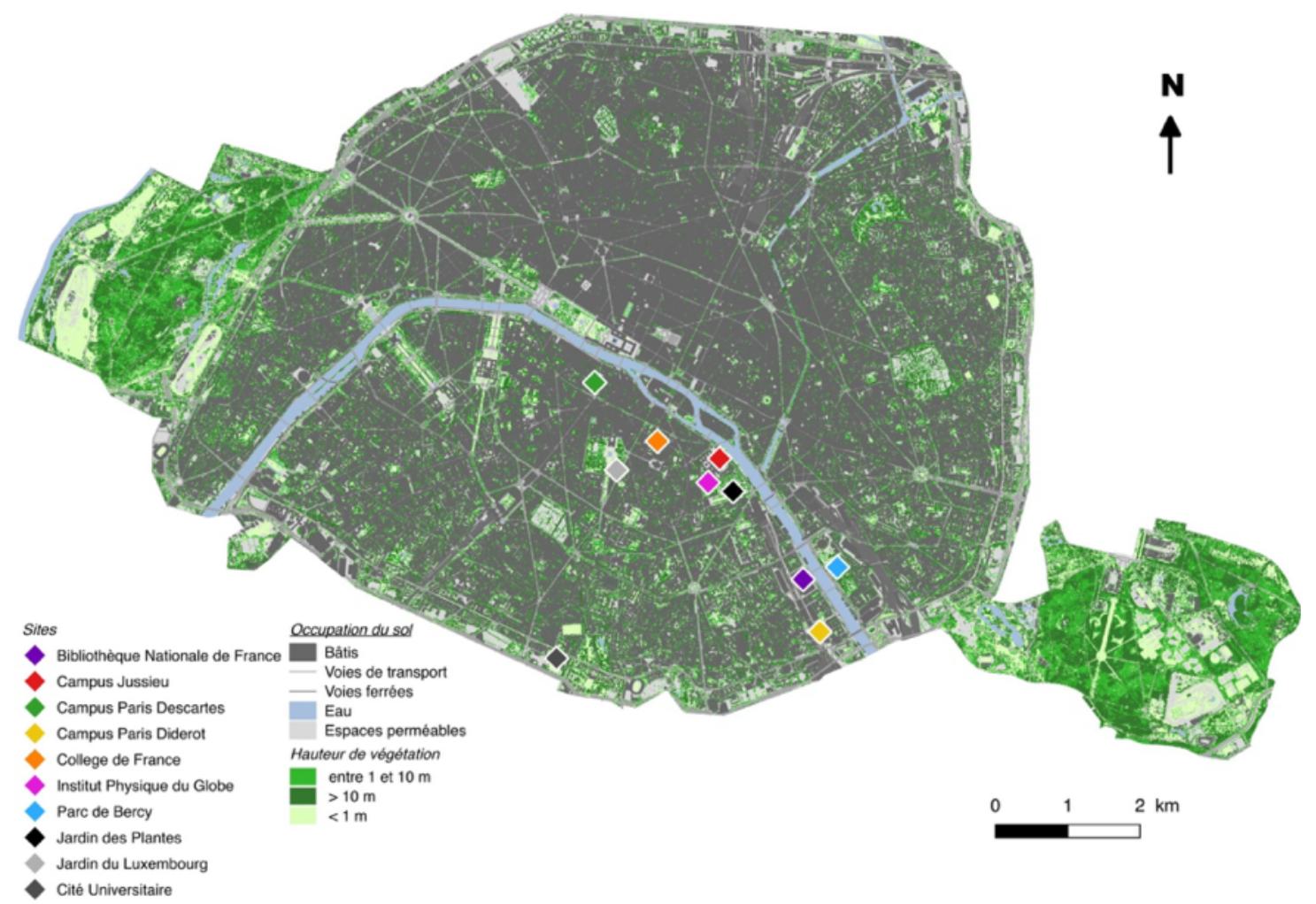

Figure $n^{\circ} \mathrm{I}$. Cartographie des sites échantillonnés. (en noir et gris, les sites de l'étude de Geslin et al. 2016 ; en couleurs, ceux de Ropars et al. 2017)

\section{Résultats}

\section{Caractérisation des abeilles parisiennes}

$\mathrm{Au}$ cours de l'année 2016, un total de 292 individus a été récolté dont 131 par piégeage passif et 161 par méthode active. Ils représentent 6 familles (Andrenidae, Apidae, Colletidae, Halictidae, Megachilidae, Melittidae), 16 genres et 55 espèces (la liste est disponible ici). A ce jour, seuls 22 spécimens du genre Hylaeus restent à déterminer. Les Halictidae dominent l'échantillon avec $33,2 \%$ de l'abondance totale suivis ensuite par les Apidae avec 29,4\% et par les Megachilidae avec 21,5\%. Les Andrènes constituent le genre le plus diversement représenté avec 23 espèces alors que seule une espèce de Sphecodes a été inventoriée. L'espèce la plus fréquemment rencontrée est Lasioglossum morio avec 31 individus soit 10,6\% du total des abeilles capturées en 2016. Elle est suivie par Anthophora plumipes avec 27 individus $(9,2 \%$ du total) et par Bombus pascuorum et Lasioglossum nitidulum avec 23 individus chacun $(7,8 \%$ du total des captures dans les deux cas). La plupart des espèces ne sont représentées que par quelques individus : 43 espèces le sont par moins de 5 individus dont 23 seulement par un seul individu. Le détail de la richesse spécifique mise en évidence par année est le suivant : 22 espèces en 2014, 43 en 2015 et 55 en 2016 amenant à un total de 74 espèces. Au total, 87 espèces d'abeilles sauvages ont été recensées jusqu'alors à Paris intramuros.

\section{Analyse des traits fonctionnels}

Parmi les 87 espèces capturées de 2011 à 2016, seulement 7 espèces sont des espèces coucous, soit $8 \%$ de la richesse spécifique et $1,6 \%$ de l'abondance. Parmi les 80 autres espèces, 12 sont oligolectiques : 6 espèces sont spécialisées pour les Asteracées ; 2 espèces pour les Fabacées ; 2 pour les Campanulacées ; 1 pour les Apiacées et une dernière pour les Borraginacées du genre Echium. Les 78 espèces restantes sont donc polylectiques dont 5 espèces possèdent tout de même des préférences pour certaines familles de plantes.

La plupart des espèces inventoriées sont solitaires $(88,7 \%), 8$ espèces $(9 \%)$ sont des bourdons au mode de vie eusocial à l'exception d'un entre eux, Bombus vestalis qui est un cleptoparasite et 2 espèces d'Halictidae sont sociales $(2,3 \%)$ : Halictus scabiosae et 

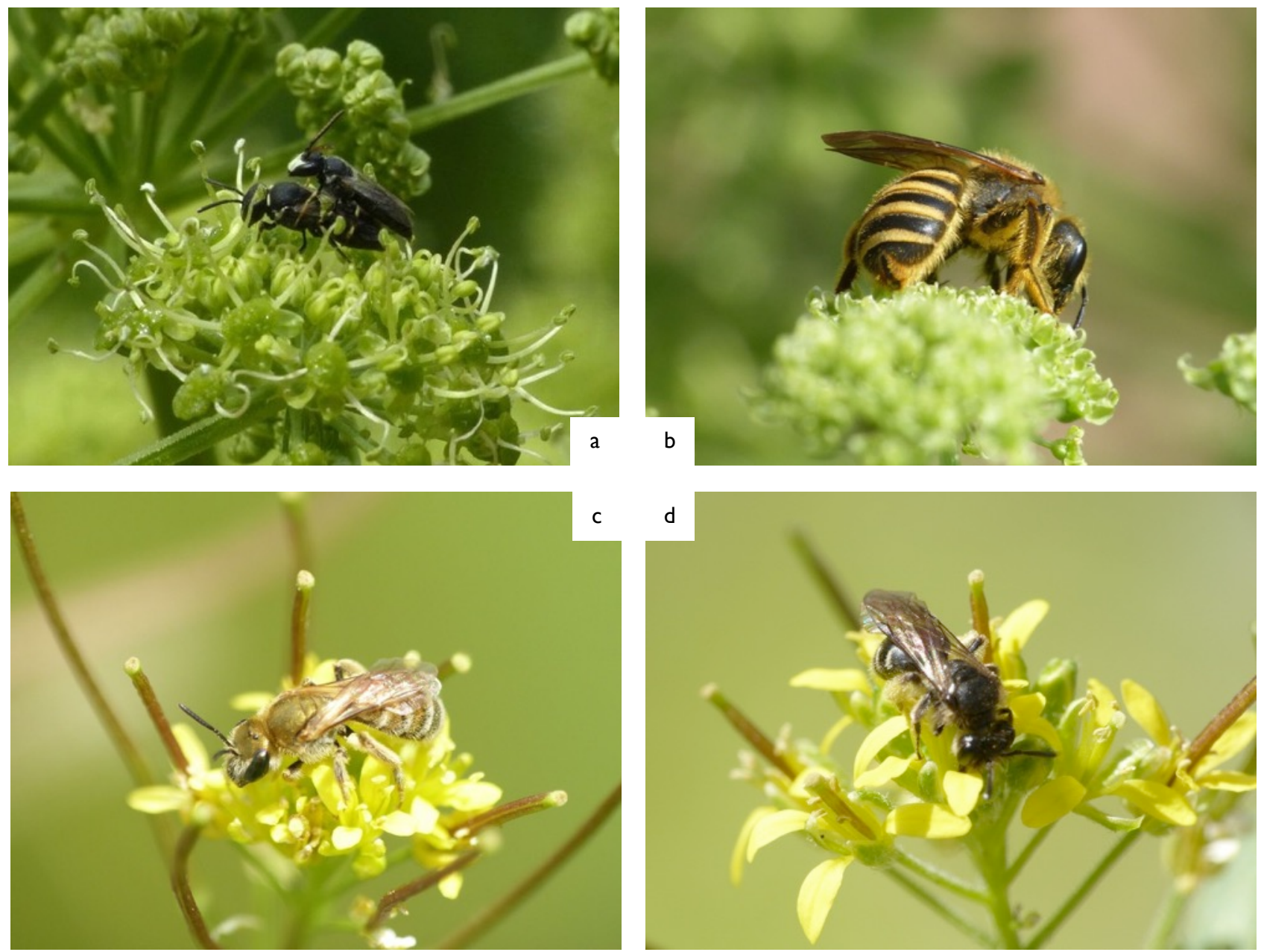

Figure $n^{\circ} 2$. (a) Couple d'Hylaeus sp.sur Angelica archangelica, (b) femelle d'Halictus scabiosae sur A. archangelica au Parc de Bercy en 2015,

(c) femelle de Seladonia sp. sur Sisymbrium irio et (d) femelle de Andrena sp. sur S. irio au Jardin des Plantes en 2015.

Lasioglossum malachurum. Sur les 80 espèces non cleptoparasites, 55 sont terricoles, 14 sont cavicoles, 8 sont rubicoles, 1 est xylocole (Xylocopa violacea). Enfin, 3 espèces peuvent nicher dans deux substrats différents.

Pour finir, seulement 3 espèces récoltées sont classées comme quasi-menacées sur la liste rouge européenne des abeilles sauvages (NIETO et al. 2014) : Lasioglossum pygmaeum, Lasioglossum sabulosum, et Lasioglossum sexnotatum. Toutes les autres espèces sont classées en préoccupation mineure (LC) ou les données qui les concernent sont insuffisantes pour statuer sur leur état de conservation (DD).

\section{Discussion}

Sur les 4 années au cours desquelles des inventaires ont été effectués dans Paris, la liste d'abeilles parisiennes totalise 87 espèces pour 1081 individus. Cela correspond à près de $9 \%$ du nombre d'espèces présentes en France, qui dépasse 970 espèces. L'ajout de la capture au filet a été bénéfique pour détecter la présence de 11 espèces supplémentaires ce qui témoigne de la nécessité de coupler les méthodes d'échantillonnages.

Avec au moins 87 espèces d'abeilles présentes à Paris, cette dernière se classe parmi les villes les plus riches en diversité derrière le Grand Lyon avec 291 espèces (ForTEL et al. 2014), Berlin avec 262 espèces (SAURE 1996), New York avec 110 espèces (FRETRIDGE et al. 2008) et enfin Poznan (Pologne) avec 104 espèces différentes (BANASZAK et al. 2011). Cette diversité existe en dépit de la faible superficie d'espaces verts dans la capitale française. En effet, les habitants de Berlin disposent de $31 \mathrm{~m}^{2}$ d'espaces verts, ceux de New York disposent de $23 \mathrm{~m}^{2}$ contre entre 5 et $14 \mathrm{~m}^{2}$ pour les habitants de Paris. Bien sûr, ces résultats doivent être interprétés avec beaucoup de prudence. Tout d'abord, il existe d'autres travaux portant sur les abeilles sauvages à Paris 
qui n'ont pas été compilés dans cet article et qui pourraient enrichir la liste d'espèces (FERRAND et al. 2014 ; StallegGer 2015 ; Plateaux 2017). De plus, les villes ne diffèrent pas seulement par leur mode d'occupation du sol mais également par leur climat, leur nombre d'habitants, leur superficie, et également par la nature de leur aire urbaine dominée ou non par des espaces agricoles. Par exemple, les disponibilités en ressources florales et en sites de nidification influencent fortement les communautés d'abeilles sauvages en ville (WRAY et al. 2014). Les espèces présentes à Paris sont majoritairement terricoles $(68,7 \%)$ mais sont proportionnellement moins représentatives que pour l'ensemble de la faune française $(80 \%$ d'espèces terricoles) ce qui suggère un effet délétère de la superficie de surfaces imperméables. Comme dans l'étude de GESLIN et al. (2016), Lasioglossum morio est l'espèce dominante sur les 4 années de suivi, représentant $20,7 \%$ du total des individus capturés, suivie de Lasioglossum nitidulum $(12,7 \%)$ et Lasioglossum laticeps $(10,4 \%)$. Nous constatons également une augmentation de la proportion d'espèces cleptoparasites par rapport aux précédentes études, cette dernière passant de $4,5 \%$ à $8 \%$ de la totalité des espèces capturées, ce qui reste encore faible par rapport aux $17 \%$ observées dans le Grand Lyon (FORTEL et al. 2014) bien que cette dernière étude présente des méthodes de captures différentes. Même si au fil des années la liste des espèces parisiennes d'abeilles sauvages s'étend, cet assemblage continue de présenter des caractéristiques d'une communauté appauvrie, tant par la dominance d'une seule espèce que par la faible proportion d'abeilles cleptoparasites.

A ce jour, il n'existe toujours pas de consensus dans la communauté scientifique sur la capacité des villes à abriter une diversité importante d'abeilles sauvages. Les espaces urbanisés semblent néanmoins être surtout propices aux espèces généralistes et ubiquistes. L'absence de consensus tient en partie au fait que pour la majorité de ces villes, les connaissances restent lacunaires. Cette étude apporte de nouvelles données pour Paris intramuros mais elle ne fait pas la synthèse de tous les travaux effectués sur la capitale française. Ceci doit être la prochaine étape dans l'amélioration de nos connaissances. Nous appelons donc ici à une mutualisation de tous ces travaux afin d'obtenir une liste plus exhaustive des abeilles parisiennes.

\section{Remerciements}

Un grand merci à David Genoud, Matthieu Aubert et Eric Dufrêne pour les déterminations à l'espèce de toutes les abeilles collectées chaque année.

\section{Bibliographie}

APUR 2004. Développer le végétal à Paris. Atelier Parisien d'Urbanisme 13.

Atlas Hymenoptera [En ligne]. Disponible à l'adresse http://www.atlashymenoptera.net (consulté le 15 février 2018).

BALDOCK K ET AL., 2015. Where Is the UK' $\mathrm{S}$ Pollinator Biodiversity? The Importance of Urban Areas for Flower-Visiting Insects. Proceedings of the Royal Society Biological Sciences 282 : 20142849.

BANASZAK-CIBICKA W, ŻMIHORSKI M, 2012. Wild bees along an urban gradient: winners and losers. Journal of Insect Conservation 16 : 331343.

Ferrand M, Garrin M, Mériguet B, GADOuM S, 2014. Réalisation d'un inventairediagnostic entomologique dans le cadre d'une démarche Oasis-nature (portée par Humanité \& Biodiversité) dans le parc de la Villette (75). Rapport final 2012-2014. Établissement public du parc et de la grande halle de la Villette (EPPGHV), Office pour les insectes et leur environnement (OPIE). $90 \mathrm{p}$.

FETRIDGE ED, Ascher JS, LANGELlotto GA, 2008. The bee fauna of residential gardens in a suburb of New York City (Hymenoptera: Apoidea). Annals of the Entomological Society of America. 101 : 1067-1077.

FlorAbeille [EN Ligne]. Disponible à l'adresse http://florabeilles.org [consulté le 15 février 2018].

FORTEL L ET AL., 2014. Decreasing Abundance, Increasing Diversity and Changing Structure of the Wild Bee Community (Hymenoptera: Anthophila) along an Urbanization Gradient. PLOS ONE 9(8).

GESLIN B ET AL., 2016. The Bee Fauna of Large Parks in Downtown Paris, France. Annales de la Société Entomologique de France 9271 (April).

Goulson D, Nicholls E, Botias C, AND Rotheray E, 2015. Bee Declines Driven by Combined Stress from Parasites, Pesticides, and Lack of Flowers. Science 347 (6229).

GENOUD D, 2017. Synthèse des connaissances sur les hyménoptères apoïdes - prélèvements 
2002 - 2009 et étude 2014. Travaux de la Massane. Tome 108.

HALl DM ET AL., 2016. The City as a Refuge for Insect Pollinators. Conservation Biology 31 (1) : 24-29. doi: 10.1111/cobi.1284.

INSEE. Population légales 2014. Commune de Paris (75056). Disponible à l'adresse https:// ww w.insee.fr/fr/statistiques/2534314? geo=COM-75056 [consulté le 15 février 2018].

NiEto A ET AL., 2014. European Red List of Bees. Publication Office of the European Union, Luxembourg. http://ec.europa.eu/environment/ nature/conservation/species/redlist/downloads/ European_bees.pdf

PotT SG ET AL., 2016. Safeguarding Pollinators and Their Values to Human Well-Being. Nature 540 : 220-229. http://dx.doi.org/10.1038/ nature20588.

Ropars L, DAJOz I, Geslin B, 2017. La ville un désert pour les abeilles sauvages ? Journal de Botanique 79 : 29-35.

Roulston H, Smith SA, Brewster AL, 2007. A comparison of pan trap and intensive net sampling techniques for documenting a bee (Hymenoptera: Apiformes) Fauna. Journal of Kansas Entomological Society 80(2) : 179-181.

SAURE C, 1996. Urban habitats for bees : the example of city of Berlin. Dans The conservation of bees (eds Matheson A, Buchmann SL, O'toole C, Westrich $\mathrm{P}$ and Williams IH), pp. 47-52. London Academic Press.

STALLEGGER P, 2015. Inventaire et analyse du peuplement d'abeilles sauvages (Hymenoptera Apoidea Anthophila) de la Petite Ceinture ferroviaire de Paris. Dans Institut d'Ecologie Appliquée \& SETEC Environnement 2015. Rapport d'étude pour la Ville de Paris, Direction des Espaces Verts et de l'Environnement. 171 p.

WESTRICH P. Habitat requirements of central European bees and the problems of partial habitats. Dans The conservation of bees (eds Matheson A, Buchmann SL, O'toole C, Westrich $\mathrm{P}$ and Williams IH), pp. 1-16. London Academic Press.

WestPhal C ET AL., 2008. Measuring Bee Diversity in Different European Habitats and Biogeographical Regions. Ecological Monographs 78(4) : 653-71.

Wilson JS, Griswold T, \& Messinger, OJ, 2008 . Sampling Bee Communities (Hymenoptera: Apiformes) in a Desert Landscape : Are Pan Traps Sufficient? Journal of Kansas Entomological Society 81(3), 288-300.

Wray JC, NeAme LA, Elle E, 2014. Floral resources, body size, and surrounding landscape influence bee community assemblages in oaksavannah fragments. Ecological Entomology 39 : 83-93.

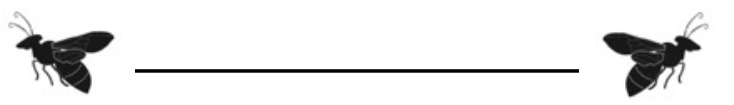




\title{
Compte rendu des captures réalisées lors de la formation Européenne à la détermination des abeilles (COST Super-B Project) dans le Parc National des Calanques
}

\begin{abstract}
par Benoît GesLIN'; Nicolas J.VereeCKeN² ; Stuart ROBERTS ${ }^{3}$;A. MURAT AYTEKIN ${ }^{4}$; David GeNOUd ${ }^{5}$; Matthieu AUBeRT ${ }^{5}$; Rosalie C. F. BURDON ${ }^{6}$; Carlos RUIZ ${ }^{7}$;Alessandro FISOGNI ${ }^{8}$; Floriane FLACHER ${ }^{1}$; Marcin GRABOWSKI ${ }^{9}$; Floriane JACQUEMIN $^{10}$; Viktor KHVIR ${ }^{11}$; Georgi KIRKITADZE ${ }^{12}$; Saskia G. T. KLUMPERS ${ }^{13}$; Karmit LEVY ${ }^{14}$; Stephanie MAHER ${ }^{15}$, Zlata MARKOV ${ }^{16}$;Adrien PERRARD ${ }^{17}$; Laura ROQUER ${ }^{18}$; Lise ROPARS' ; Lucie SCHURR ${ }^{1}$;AndriVARNAVA ${ }^{19}$; \& Denis MICHEZ ${ }^{20}$
\end{abstract}

\section{Introduction}

A l'échelle Européenne, le pourtour méditerranéen français représente un haut lieu de la biodiversité des abeilles sauvages. En effet, sur près de 2000 espèces répertoriées par la Liste Rouge UICN des abeilles sauvages d'Europe (NIETO et al., 2014), on estime que plus de 700 espèces seraient présentes sur une zone allant des Pyrénées Atlantiques aux contreforts Alpins. Cependant, ce chiffre ne reste qu'une estimation de la réelle richesse spécifique des abeilles autour du littoral méditerranéen, pour deux raisons principales. La première raison, soulignée par l'étude de NIETO et al. (2014), tient dans le fait que pour plus de $55 \%$ des 2000 espèces répertoriées en Europe, nous ne disposons pas, actuellement, de suffisamment de données pour pouvoir statuer sur l'état réel de leurs populations ou de leur répartition géographique. La seconde raison réside dans le fait qu'à l'échelle locale la plupart des

\footnotetext{
(1)Aix Marseille Univ, Univ Avignon, CNRS, IRD, IMBE, Aix-Marseille Université - Campus Étoile Faculté des Sciences St-Jérôme Case 42I Av Escadrille Normandie Niémen 13397 Marseille Cedex 20, France

(2)AgroecologyLab, Université libre de Bruxelles (ULB), Boulevard du Triomphe CP 264/2, B-I050 Bruxelles, Belgique.

${ }^{(3)}$ School of Agriculture, Policy and Development, University of Reading, Reading, UK

(4) Hacettepe University Faculty of Science Department of Biology 06800 Beytepe/Ankara/Turkey

(5)Observatoire des Abeilles, Arzens, France

(6) Department of Plant Ecology and Genetics, Evolutionary Biology Centre, Uppsala University, Norbyvägen I8D Uppsala, 75236, Sweden

(7)Dpto. Zoología y Antropología Física, Facultad de Veterinaria, Universidad de Murcia, 30100 Murcia, Spain.

(8)Univ. Lille, CNRS, UMR 8198 - Evo-Eco- Paleo, F-59000 Lille, France

(9)Department of Applied Entomology, Faculty of Horticulture, Biotechnology and Landscape

Architecture, Warsaw University of Life Sciences, Nowoursynowska 159, 02-776 Warsaw,

Poland

(10)Université de Liège-Gembloux Agro-Bio Tech, Unité de recherche BIOSE (ingénierie des

bio-systèmes), Axe Biodiversité et Paysage, Passage des Déportés 2, B-5030 Gembloux,

Belgique.

(II)Faculté de biologie, Université d'Etat de Biélorussie, Minsk, Biélorussie

(12)Agricultural University of Georgia, Institute Of Entomology, Kakha Bendukidze University Campus, \# 240 David Aghmashenebeli Alley, Tbilisi, Georgia

(13)Plant Ecology and Phytochemistry, Institute of Biology Leiden, Leiden University, Sylviusweg 72, 2333 BE Leiden, The Netherlands

(14) Department of Entomology The Robert H Smith Faculty of Agriculture, Food and Environment and The Advanced School for

Environmental Studies, The Hebrew University of Jerusalem

(15)Dept. of Biology, Anglia Ruskin University, Cambridge, UK.

(16) University of Novi Sad, Faculty of Science, Department of Biology and Ecology, 21000 Novi Sad, Serbia

(17)Univ Paris Diderot, iEES Paris UMR 7618, CNRS, Sorbonne Université, Sorbonne Paris Cité, 4 Place Jussieu, 75005, Paris, France

(18)CREAF, Universitat Autònoma de Barcelona, 08193 Cerdanyola del Vallès, Spain

(19)Cyprus University of Technology, Department of Agricultural Sciences, Biotechnology and Food Science, 30 Archbishop Street, 3036 Limassol/Cyprus

(20)Université de Mons, Institut de recherche des Biosciences, Laboratoire de Zoologie, place du parc 23, 7000 Mons, Belgique
}

OSMIA $\mathrm{N}^{\circ} 7$ 
gestionnaires ignorent la biodiversité des abeilles qui vivent réellement sur leurs territoires. Ces deux raisons expliquent principalement les manques qui existent dans les bases de données Européennes.

A titre d'exemple, la base de données du Parc national des Calanques comptait en 2016 moins d'une dizaine espèces d'abeilles dont Apis mellifera et Bombus terrestris. Ce simple constat laissant penser que peu d'études avaient alors été réalisées dans cette zone. C'est une des raisons pour laquelle nous avons décidé d'organiser en 2016 une campagne de collecte et de détermination d'abeilles dans le cadre d'une formation des doctorants/postdoc européens travaillant sur les questions liées aux thématiques de pollinisation (programme Européen COST-Super B ci-après Bee Course http://www.superb-project.eu/, figure 1). Notre objectif principal était de réaliser trois journées de collecte au sein du Parc National des Calanques et ainsi d'apporter de nouvelles informations sur la faune locale d'Apoidea Apiformes.

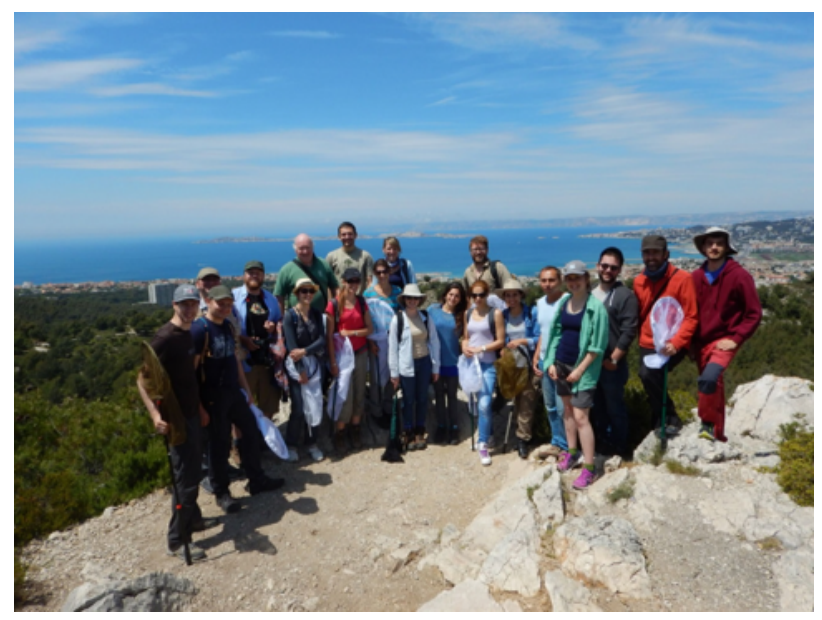

Figure I. Diversité des observateurs du Bee Course 2016. Photographie : N.Vereecken.

Cet article présente les résultats de cette étude de terrain en exposant $1 /$ les différentes espèces capturées, 2/ leur statut de conservation au regard de la liste rouge de l'UICN, et 3/ leurs traits d'histoire de vie.

\section{Méthodes}

Sites

Le Parc National des Calanques (ci-après " PNCal») est le $10^{\mathrm{e}}$ et le plus récent (2012) Parc national français (figure 3 ). Il est le premier parc national périurbain d'Europe. Il s'étend à la fois entre terre et mer, son cœur marin couvrant 435 $\mathrm{km}^{2}$ et son cœur terrestre $85 \mathrm{~km}^{2}$. En terme pédologique, le massif des Calanques est constitué principalement de roches calcaires (ElLILI et al., 2017). Sa flore est bien connue des botanistes (plus de 800 espèces végétales, VÉLA et al., 2001 ; D. Pavon com. pers.). L'habitat majoritaire du PNCal est la garrigue écorchée ou garrigue à cistes composée en majorité de Ciste blanc (Cistus albidus L.), de Ciste de Montpellier (Cistus monspeliensis L.), de Romarin (Rosmarinus officinalis L.) et de Thym (Thymus vulgaris L.) (figure 2). En revanche, la faune d'arthropodes terrestres et notamment d'abeilles sauvages reste très peu documentée. Au sein du PNCal, l'échantillonnage de la Bee Course fut déployé sur trois sites : le campus de Luminy $\left(43^{\circ} 13^{\prime} 52^{\prime \prime} \mathrm{N} ; 05^{\circ} 26^{\prime} 22^{\prime \prime} \mathrm{E}\right)$; la montagne de l'Aigle $\left(43^{\circ} 13{ }^{\prime} 59^{\prime \prime} \mathrm{N} ; 05^{\circ} 23^{\prime} 16^{\prime \prime} \mathrm{E}\right)$ et le vallon du Logisson $\left(43^{\circ} 14^{\prime} 07^{\prime \prime} \mathrm{N} ; 05^{\circ} 30^{\prime} 02^{\prime \prime} \mathrm{E}\right.$ ) (voir figure $3)$.

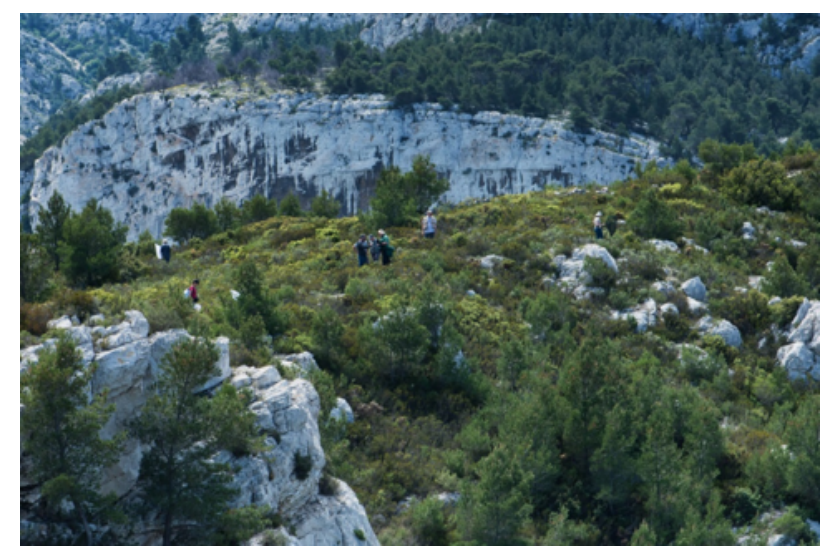

Figure 2. Garrigue écorchée, milieux caractéristique du Parc des Calanques. Photographie : N.Vereecken.

\section{Captures}

Les captures furent réalisées le mardi, mercredi et jeudi 17-18-19 mai 2016. Le temps était alors beau et sec $\left(>15^{\circ} \mathrm{C}\right.$, pas de couverture nuageuse). Ces captures ont été réalisées au filet à papillon ainsi qu'à l'aide d'aspirateurs à bouche selon la méthode de la chasse active par les 20 participants à la formation. Les spécimens ont été étalés le jour même de la capture puis identifiés par des taxonomistes spécialistes de chacun des groupes : Matthieu Aubert (Eucera), Alain Pauly (Halictidae), David Genoud (Andrena), Holger Dathe (Hylaeus), Mickael Kuhlman (Colletes), Michel Terzo (Ceratina), Sebastien Patiny (Panurgus), Pierre Rasmont (Bombus, Melecta et Anthophora) \& Maximilian 


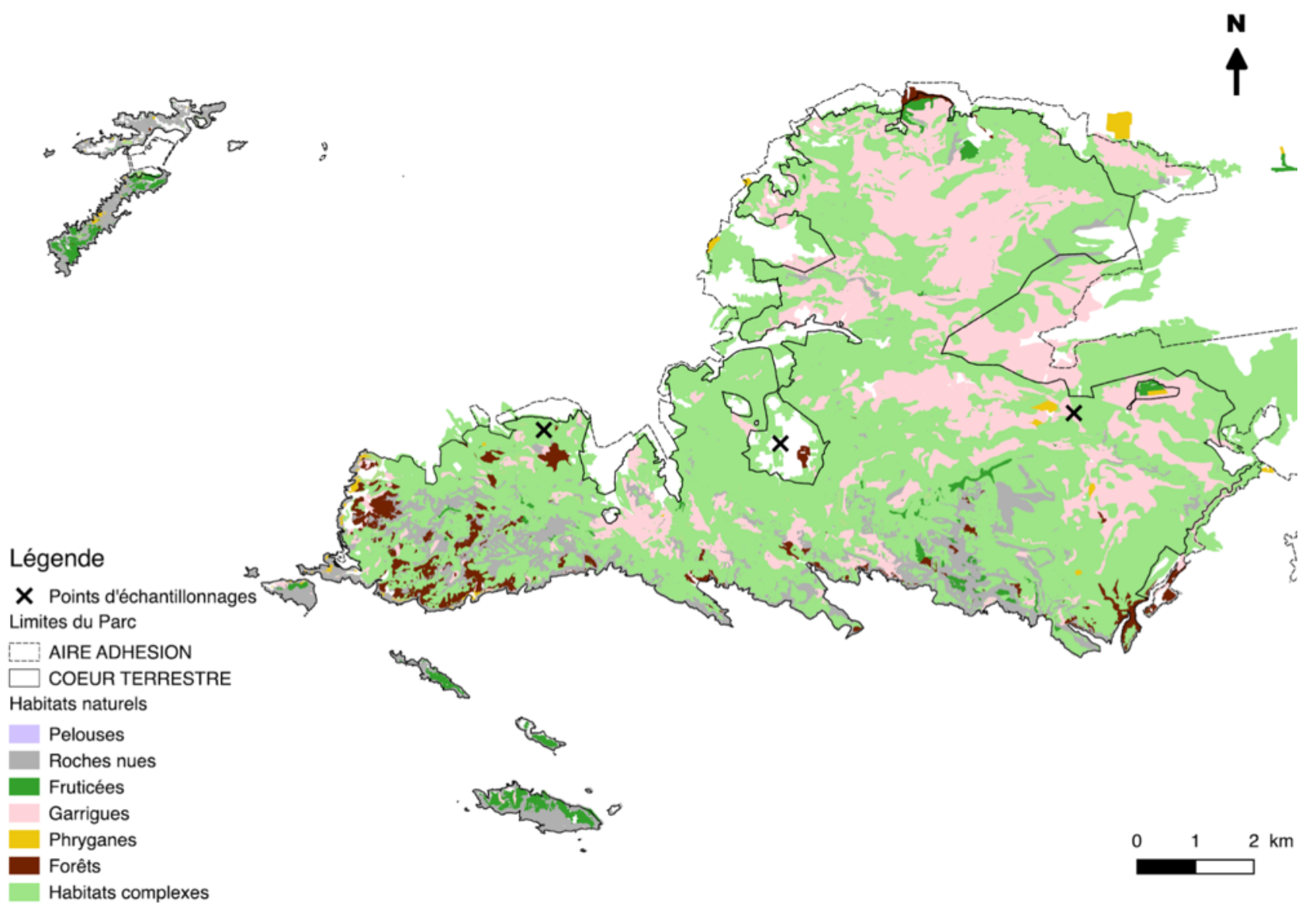

Figure 3. Carte du Parc National des Calanques.

Schwarz et Eric Dufrêne (Nomada). Les traits d'histoire de vie des espèces capturées (lectisme, socialité et substrat de nidification) ont été compilés à partir de la littérature (CHOREIN, 2007 ; CARRÉ, 2008 ; FORTEL, 2014 ; COIFFAITGOMBAUlT et al., 2016 ; GENOUd, 2017 ; MAGHNI et al., 2017) et des bases de données en ligne (de JONG et al., 2014 ; Bees, Wasps \& Ants Recording Society, 2016 ; Atlas Hymenoptera) et par les coauteurs eux-mêmes.

\section{Résultats}

Pour plus de précision sur les données, le lecteur est invité à se référer à l'intégralité de la base de données qui est accessible en ligne sur le site de l'Observatoire des abeilles ici.

\section{Abondance et richesse}

$\mathrm{Au}$ total, 586 individus ont été capturés (figure 4). Ils appartiennent aux familles Andrenidae (105 spécimens), Apidae (75 spécimens), Colletidae (67 spécimens), Halictidae (124 spécimens), Megachilidae (213 spécimens) et Melittidae (2 spécimens - à noter que le genre Dasypoda est classé au sein des Melittidae sensu lato suivant les recommandations de DANFORTH et al., 2013). Le genre le plus représenté est le genre Andrena (79 spécimens), suivi par le genre Hylaeus (60 individus).

Sur ces 586 individus, 103 espèces ont été répertoriées, la plus grande diversité se situant chez les Megachilidae.

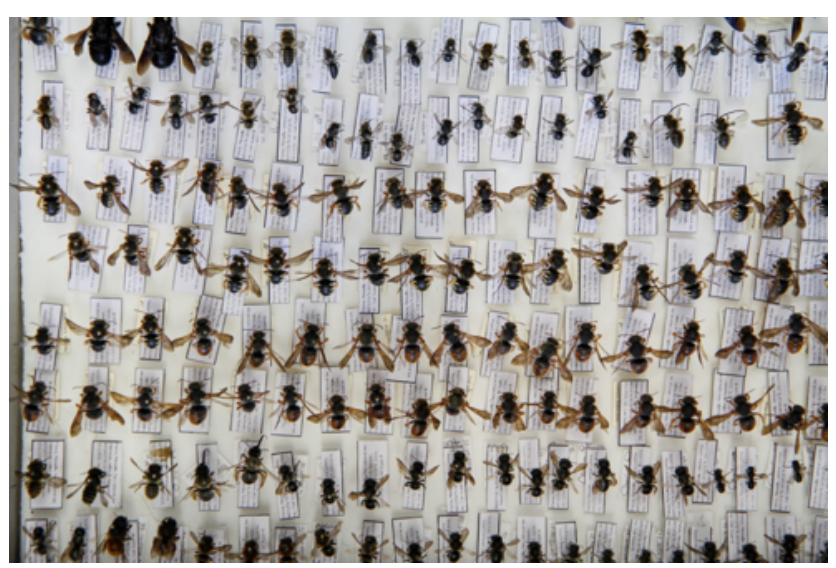

Figure 4. Diversité des spécimens échantillonnés dans le Bee Course 2016. Photographie : L. Schurr. 
Andrenidae (18 espèces) : Andrena agilissima, $A$. brumanensis, A. cinerea, A. combinata, $A$. curtula, A. fabrella, A. flavipes, A. lagopus, $A$. morio, A. nigroaenea, A. niveata, A. ovatula, $A$. pusilla, A. similis, A. simontornyella, $A$. tenuistriata, A. truncatilabris, Panurgus dentites; Apidae (21 espèces): Apis mellifera, Bombus pascuorum, B. ruderatus, B. terrestris, Eucera caspica, E. hispana, E. nigrifacies, Nomada discrepans, N. femoralis, N. glaucopis, $N$. integra, N. rubricoxa, Xylocopa violacea, Anthophora aestivalis, A. femorata, A. mucida, A. plumipes, Melecta albifrons, M. italica, Ceratina chalcites, C. dentiventris.

Colletidae (12 espèces) : Colletes nigricans, Hylaeus brachycephalus, H. clypearis, $H$. euryscapus, H. hyalinatus, H. imparilis, $H$. lineolatus, $H$. pictipes, $H$. pictus, $H$. punctatus, $H$. signatus, $H$. variegates.

Halictidae (23 espèces) : Halictus fulvipes, $H$. maculatus, H. quadricinctus, $H$. scabiosae, $H$. simplex, Lasioglossum albocinctum, L. bimaculatum, L. corvinum, L. glabriusculum, L. griseolum, L. interruptum, L. leucozonium, $L$. malachurum, L. nigripes, L. pauperatum, $L$. pauxillum, L. politum, L. pygmaeum, L. transitorium planulum, L. villosulum, Seladonia gemmea, S. smaragdula sensu lato, Sphecodes ruficrus.

Megachilidae (26 espèces) : Anthidium manicatum, Coelioxys afra, Hoplitis adunca, $H$. anthocopoides, $H$. benoisti, $H$. bisulca, $H$. cristatula, Megachile apicalis, M. melanopyga, M. parietina, M. pilidens, M. willughbiella, Osmia aurulenta, O. brevicornis, O. latreillei, $O$. ligurica, O. melanogaster, O. niveata, $O$. scutellaris, O. signata, O. submicans, $O$. tricornis, O. versicolor, Rhodanthidium septemdentatum, R. sticticum, Stelis signata.

Melittidae (2 espèces) : Dasypoda argentata, Dasypoda cingulata.

\section{Statut de conservation}

Au regard de la Liste Rouge UICN des abeilles européennes (NIETO et al., 2014), quatre espèces d'abeilles considérées comme quasi-menacées (Near-Threatened) ont été capturées : Andrena ovatula, Halictus quadricinctus, Lasioglossum pygmaeum (deux individus), et Dasypoda argentata. Une nouvelle espèce pour la région
PACA a été capturée : Osmia latreillei. Enfin, il est à noter que nous faisons ici mention pour la première fois pour la France de la capture d'un individu de Nomada rubricoxa (SCHWARZ, 1977). Cette femelle a été capturée le 19 mai 2016 sur le site du campus de Luminy. La première mention de cette espèce en France fera l'objet ultérieurement d'une publication indépendante incluant sa description complète.

\section{Traits d'histoire de vie}

En ce qui concerne les préférences alimentaires des espèces identifiées, nous avons répertorié 33 oligolectiques (32\% de la richesse spécifique). Quarante-cinq espèces ont été classées comme polylectiques s.s. (43\%); 12 espèces supplémentaires sont considérées également polylectiques mais avec des préférences particulières pour certaines familles de plantes (voir base de données). Enfin 11 espèces sont non renseignées. L'oligolectisme le plus courant porte sur les Astéracées (14 espèces). La socialité et le grégarisme sont moins représentés parmi nos individus (17 espèces - 16\%) que la nidification solitaire (73 espèces - 70\%, figure 5 ) tandis que nous manquons de précisions pour 9\% d'entre eux. Il est à noter que 51 espèces (49\%) présentent un comportement de nidification terricole, et que $28(27 \%)$ utilisent préférentiellement des cavités pour nidifier (voir la base de données pour plus de précisions). Pour ces dernières, il est intéressant de voir que quelques espèces telles que Osmia aurulenta ou encore Rhodanthidium sticticum sont hélicicoles. Enfin, 26 individus ( $0.5 \%$ de l'abondance totale) appartenant à neuf espèces cleptoparasites ( $8 \%$ de la richesse) ont été identifiés.

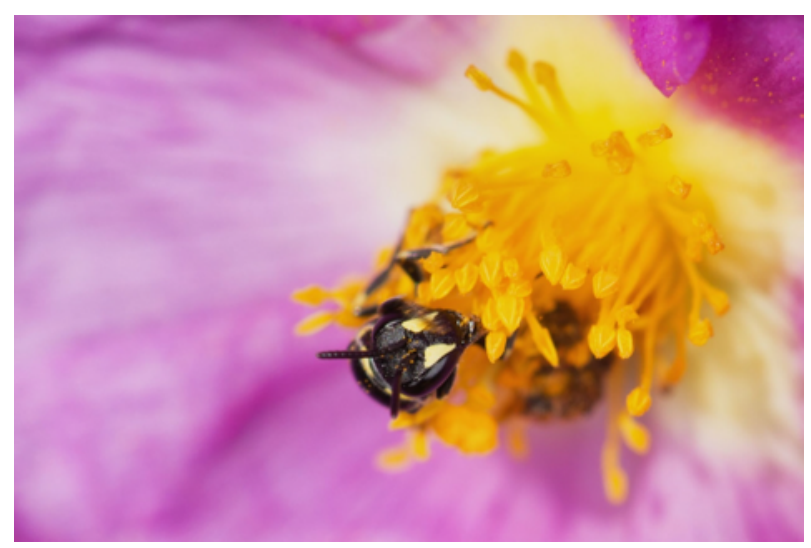

Figure 5. Femelle d'une espèce caulicole (Hylaeus sp.) en train de se nourrir. Photographie : N.Vereecken. 


\section{Discussion}

Un effort d'échantillonnage relativement modéré a permis d'augmenter de manière significative le nombre d'espèces recensées dans le PNCal. Cette étude illustre ainsi les lacunes dans les connaissances relatives à la diversité des abeilles sauvages du Parc. Outre cette étude, peu de données existent à notre connaissance sur le territoire des Calanques en dehors de quelques captures opportunistes ou de collections anciennes. En 2009, au cours de quelques journées de capture au sein du territoire du PNCal, X. Lair avait notamment capturé Lasioglossum soror classée en danger d'extinction ("EN ") par l'UICN, mais ces données n'ont pas été publiées. Des travaux comme ceux réalisés par l'Observatoire des Abeilles dans le Parc National du Mercantour (Inventaire des abeilles sauvages (Hymenoptera : Apoidea : Anthophila) dans le Parc National du Mercantour en 2012, 2013) ou par C. CoiffaitGombault dans le Parc National de Port-Cros (Coiffait-Gombault et al., 2016) sont nécessaires à l'établissement d'une liste d'espèce représentative du PNCal. C'est notamment un des objets de la thèse de doctorat de L. Ropars actuellement en cours.

Ces résultats encourageants ne peuvent former qu'un état des lieux préliminaire à l'évaluation de la diversité des abeilles sauvages dans le PNCal : les captures n'ont été réalisées que sur 3 jours, sur 3 sites, par chasse active d'observateurs plus ou moins expérimentés dans l'échantillonnage, du niveau débutant au niveau le plus chevronné. Or la méthode d'échantillonnage comme la saison de collecte affectent l'observation de la diversité des abeilles (WestPhAL et al., 2008). Nous n'avons donc ici qu'effleuré la diversité réelle des abeilles sauvages dans le PNCal. Les futures campagnes de captures devront être réalisées sur toute la saison de butinage (de février à août et éventuellement en automne), en augmentant le nombre de sites d'échantillonnage et en combinant différentes méthodes de captures (filet + coupelles par exemple).

En seulement trois journées de captures, nous pouvons tout de même noter que la richesse spécifique capturée est tout à fait remarquable puisqu'elle représente plus de $10 \%$ de la faune française d'abeilles sauvages (RASMONT et al., 1995). De plus, cette première campagne de capture présume d'une diversité bien plus importante, notamment au regard de la liste rouge de l'UICN qui évalue la richesse spécifique à près de 700 espèces sur le pourtour méditerranéen français (NIETO et al., 2014). Cependant, l'abondance d'abeilles cleptoparasites semble étonnamment faible aux vues du caractère protégé du PNCal $(0.5 \%$ de l'abondance) et de la publication de Sheffield et ses collaborateurs (SHEFFIELD et al., 2013) qui présentaient ce groupe comme indicateur de la bonne santé d'un écosystème. Ce résultat confirme d'autant plus la nécessité de réaliser de nouvelles investigations dans le PNCal.

L'étude réalisée lors de la Bee Course préfigure ainsi des études à venir et pose les premiers jalons d'une estimation réaliste de la biodiversité d'abeilles sauvages dans le PNCal. Les futures études permettront au $10^{\mathrm{e}}$ Parc national Français de mieux connaître les espèces qui vivent sur son territoire et de renforcer ainsi les mesures de protection envers sa faune sauvage et ses habitats qui doivent faire face à des pressions croissantes.

\section{Remerciements}

Nous tenons à remercier les spécialistes qui ont aidé à déterminer jusqu'au niveau spécifique les spécimens capturés : Mathieu Aubert pour les Eucera et les Megachilidae, Eric Dufrêne et Maximilian Schwarz pour les Nomadinae, David Genoud pour les Andrena, Sébastien Patiny pour les Panurgus, Michael Kuhlmann pour les Colletes, Holger Dathe pour les Hylaeus, Alain Pauly pour les Halictidae, Pierre Rasmont pour les Bombus, Melecta et Anthophora, Michael Terzo pour les Ceratina.

\section{Bibliographie}

ChOREIN A, 2007. Systématique et chorologie des Anthophorini (Hymenoptera: Apidae) de Belgique et du Nord de la France, avec une première analyse de leurs sécrétions volatiles. Mémoire de Licence, 86p.

DE JoNG et al., 2014. Fauna Europaea - all European animal species on the web. Biodivers. Data J. 2, e4034. doi:10.3897/BDJ.2.e4034

GENOUD D, 2017. Synthèse des connaissances sur les hyménoptères apoïdes. Prélèvements 2002 - 2009 et étude 2014. Travaux de la Massane, Tome $\mathrm{n}^{\circ} 108$ 
Illili A, Rabier J, Prudent P, SAlducci MD, HECKENROTH A, LACHAAL M, LAFFONTSchwoB I, 2017. Decision-making criteria for plant-species selection for phytostabilization: Issues of biodiversity and functionality. $J$. Environ. Manage. 201, 215-226

FORTEL L, 2014. Écologie et conservation des abeilles sauvages le long d'un gradient d'urbanisation. Thèse de Doctorat. Université d'Avignon.

CARRÉ G, 2008. Biodiversité, paysages et conservation de la communauté d'abeilles dans les agrosystèmes. Thèse de Doctorat. Université d'Avignon.

Coiffait-Gombault C, Crouzet N, Morisson N, Guilbaud L, VAISSIÈre B, 2016. Diversité des abeilles sauvages (Hymenoptera: Apoidea) de l'île de Porquerolles (France, Var). Sci. Rep. Port-Cros natl. Park 143, 95-143.

MAGHNi N, LOUAI K, ORTIZ-SANCHEZ FJ, RASMONT P, 2017. Les Anthophores de la région des Aurès (nord-est de l'Algérie) (Hymenoptera : Apidae : Anthophorini). Ann. la Soc. Entomol. Fr. 53, 55-73. doi:10.1080/00379271.2017.1305916

NIETO A ET AL., 2015. European Red List of Bees, Luxembourg: Publication Office of the European Union. doi:10.2779/77003

RAsmont P, Ebmer A, Banaszak J, VAN Der ZANDEN G, 1995. Hymenoptera Apoidea Gallica. Liste taxonomique des abeilles de France, de Belgique, de Suisse et du Grand-Duché de Luxembourg, Bull. Soc. Entomol. Fr. 100 (hors série), 1-98

Sheffield CS, Pindar A, Packer L, Kevan PG, 2013. The potential of cleptoparasitic bees as indicator taxa for assessing bee communities. Apidologie 44, 501-510. doi:10.1007/ s13592-013-0200-2

Vela E, Pavon D, Giraud R, Destefano M. \& SAATKAMP A, 2001. Liste des plantes vasculaires du site classé des Calanques (Marseille/Cassis). Bulletin de la Société linnéenne de Provence, 52 : 139-148.

WestPhaL C, et al., 2008. Measuring bee diversity in different European habitats and biogeographical regions. Ecol. Monogr. 78, 653671. doi:10.1890/07-1292.1
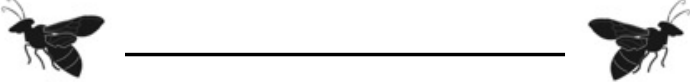


\title{
Présence de \\ Bombus argillaceus SCOPOLI 1763 (Apoidae - Apidae - Bombini) dans les Hautes-Alpes en France
}

\author{
par Bernard FRIN*
}

Résumé. La difficulté qui existe dans la nature pour déterminer les espèces de Bombus parmi les espèces françaises m'a amené à choisir les reines de Bombus argillaceus, espèce polylectique, remarquable et facile à identifier même sur photo. Cette espèce atteint sa limite occidentale de distribution géographique dans nos vallées alpines où sa présence et sa répartition sont mal connues. Les résultats de cette enquête menée essentiellement sur les saisons 2013 et 2014 montrent que Bombus argillaceus (Scopoli 1763) est bien présent dans toutes les vallées des Hautes-Alpes, de l'étage collinéen à l'étage montagnard jusqu'à $1700 \mathrm{~m}$ environ. L'espèce est aussi attestée dans la vallée de l'Ubaye dans les Alpes-de-Haute-Provence. Seuls les secteurs du Serrois, du Devoluy, du Briançonnais et du Queyras sont restés vierges d'observations à ce

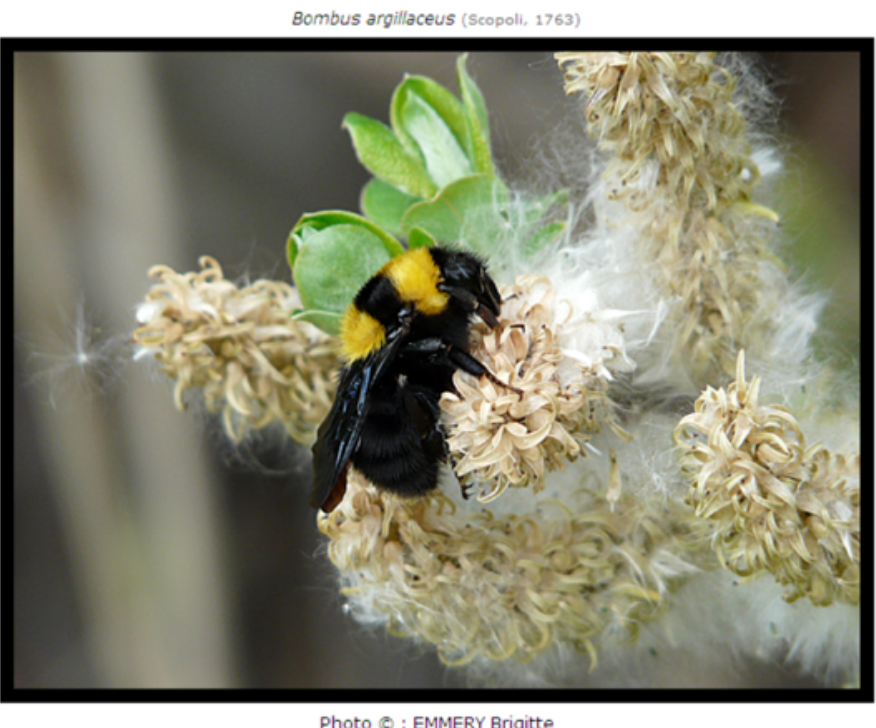

Figure I. Bombus argillaceus sur Salix sp. (Salicaceae). jour. Ceci est sans doute dû à une sousprospection de ces parties des Alpes françaises.

\begin{abstract}
As Bombus species are difficult to identify to the species level to the naked eyes, I chose to focus my observations on the queens of Bombus argillaceus among the French species. This is a remarkable species able to feed on various plants species (polylectic) and easily recognisable even on pictures. This species reaches its western limit in alpine valleys of France where its presence and distribution are not very well known. The results of our field prospections, mostly conducted during the 2013 and 2014 seasons, show that this species is well established in all the High-Alps valleys, as well as in the Ubaye Valley (Alpes-de-Haute-Provence) - up to about 1700 meters. To this day, we don't have any data in the areas of Serre, Devoluy, Queyras and Briançon, probably due to the low sampling effort in these regions.
\end{abstract}

\section{Introduction}

Je fus fort intrigué lorsque pour la première fois (le 9 avril 2009 à Châteauroux-les-Alpes) j'ai rencontré ce magnifique bourdon. Mon esprit s'est mis à vagabonder, me faisant tout d'abord penser avec ses ailes noires à une espèce de

*29 rue des Vignes, 05200 Embrun - be.frin@wanadoo.fr
Xylocopa, puis à un bourdon " coucou ». Rentré chez moi, je pus, après quelques recherches, enfin mettre un nom sur le spécimen capturé : Bombus argillaceus (figures 2 et 3). Membre du GRENHA (GRoupement des ENtomologistes des Hautes-Alpes) depuis sa création, je propose en septembre 2009 de commencer une collection du genre Bombus dans le département afin d'en faire l'inventaire. Depuis cette période, j'ai 


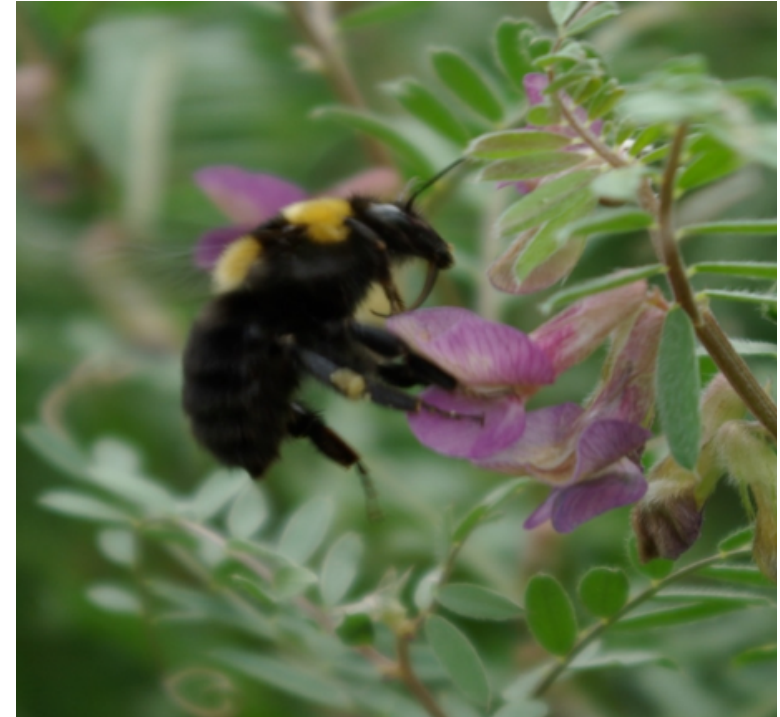

Figure 2. Bombus argillaceus sur Vicia pannonica (Fabaceae). (Photo : B. Frin)

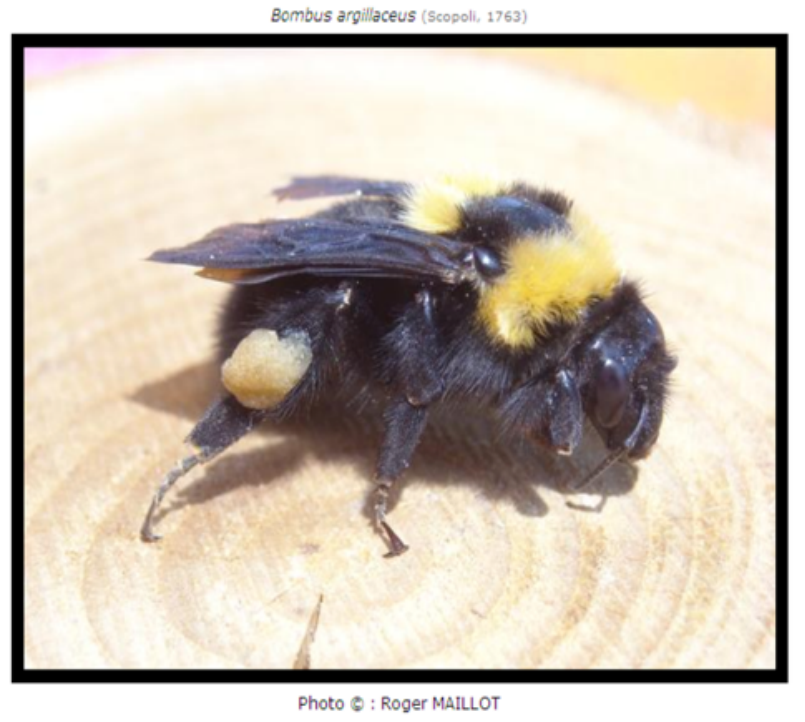

Figure 3. Reine Bombus argillaceus avec pelotes.

réalisé plein de belles découvertes dans ce genre mais également dans beaucoup d'autres genres d'Apoidea et ma collection compte actuellement 37 espèces de Bombus. C'est dans ce contexte que je suis aussi devenu membre et acteur de l'Observatoire des Abeilles (OA). Dans ce récit naturaliste, je vous présente certaines de mes observations autour de l'aire de répartition de Bombus argillaceus dans les vallées alpines françaises.

\section{Matériel et méthodes}

Début 2013, je décide de lancer une enquête sur Bombus argillaceus dans le département des Hautes-Alpes. A cet effet, je sollicite toutes mes relations naturalistes pour noter avec précision toutes leurs observations de reine de cette espèce et prendre des clichés. Les outils utilisés sont la clé des bourdons du massif armoricain par Gilles MAHÉ (2011) et la clé de détermination du Pr. Pierre RASMONT. J'ai également beaucoup utilisé le site Atlas Hymenoptera (RASMONT P. et ISERBYT S. 2010-2013). Des spécimens de références sont présents dans ma collection personnelle dans laquelle chacune des identifications ont été contrôlées par G. Mahé. L'ensemble des données sont disponibles puisque la saisie de toutes les observations a été faite dans la base de données du GRENHA, de laquelle sont extraits les documents qui sont inclus dans cette enquête.

\section{Pattern}

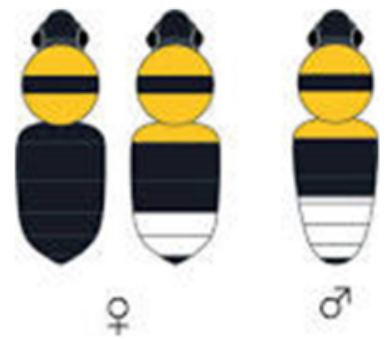

Figure 4. Pattern chez Bombus argillaceus.

\section{Résultats}

La consultation des deux cartes (figures 5 et 6 ) ci-dessus et la répartition altitudinale (figure 7)

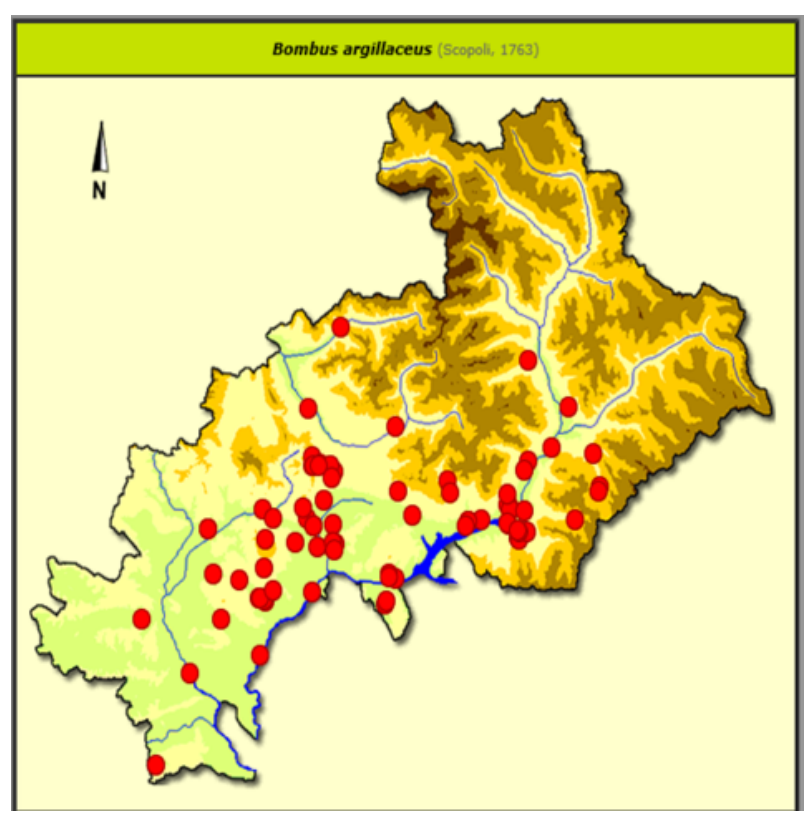

Figure 5. Observations dans les bassins fluviaux. 


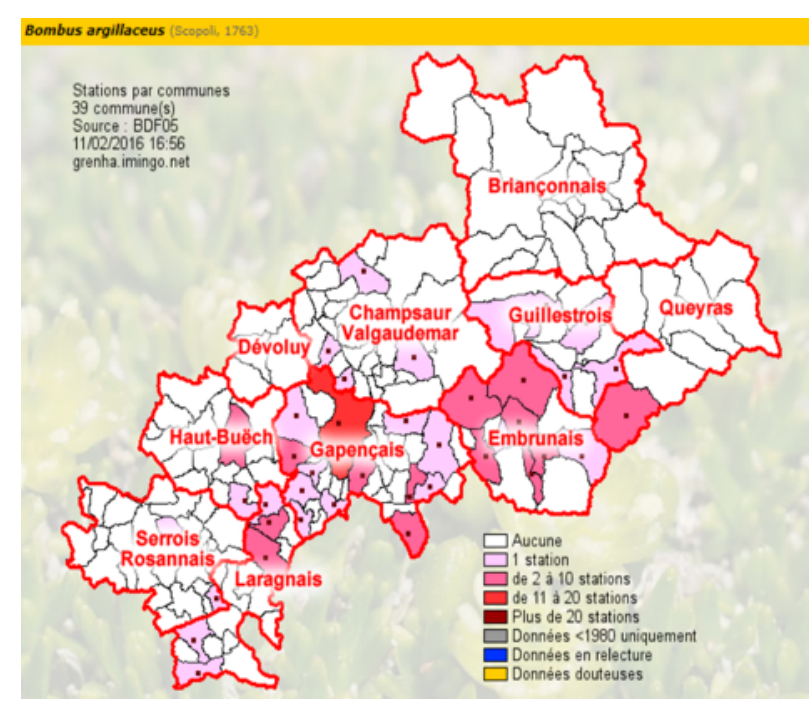

Figure 6. Communes et secteurs des Haute-Alpes.

permettent d'affirmer que $B$. argillaceus est bien présent dans toutes les vallées des HautesAlpes : Durance, Buëch, Drac (à un degré moindre) et celle de l'Ubaye dans le département voisin, les Alpes- de-Haute-Provence.

Les 92 observations enregistrées dans la carte communale (figure 6) couvrent 39 communes sur les 177 qui constituent le département. Les communes restées vierges d'observations et qui sont entourées par d'autres communes ou l'espèce a été observée, sont très certainement occupées bien que cela reste à vérifier car ces absences sont sûrement dû à un manque de pression d'observation. L'espèce occupe tout l'étage collinéen ainsi que l'étage montagnard dans sa partie, peu ou non boisée (l'observation la plus haute se situe à $1710 \mathrm{~m}$ ).

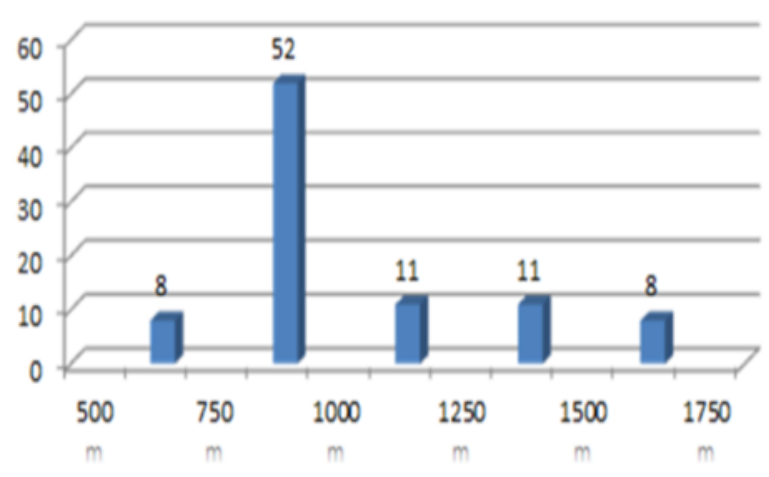

Figure 7. Répartition altitudinale des observations. Point le plus bas du département : 470 m Ribiers.

Enfin, nous pouvons noter l'absence d'observations de $B$. argillaceus dans les zones amont de la vallée de la Durance au nord, le
Briançonnais ainsi que dans le Queyras (haute vallée du Guil) puis à l'ouest dans le Serrois et le Dévoluy. Cela n'exclut pas la présence de l'espèce sur ces secteurs qui ont été très largement sous prospectés.

\section{Phénologie}

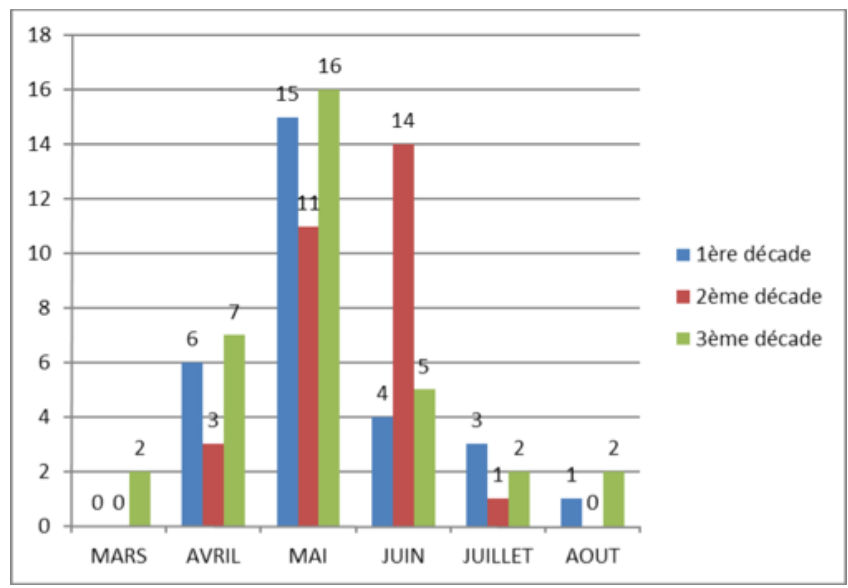

Figure 8. Phénologie de Bombus argillaceus.

L'émergence de l'espèce débute vers la fin mars (l'observation la plus précoce le 26.III.2011). Les observations continuent jusqu'à la deuxième décade de juin avec la plus grande partie (42 d'entre elles) au cours du mois de mai. L'observation la plus septentrionale a été réalisée sur la commune de Freissinières, au lieu-dit Les Aujards (4445’56.2” N, 6³1’34.3”E).

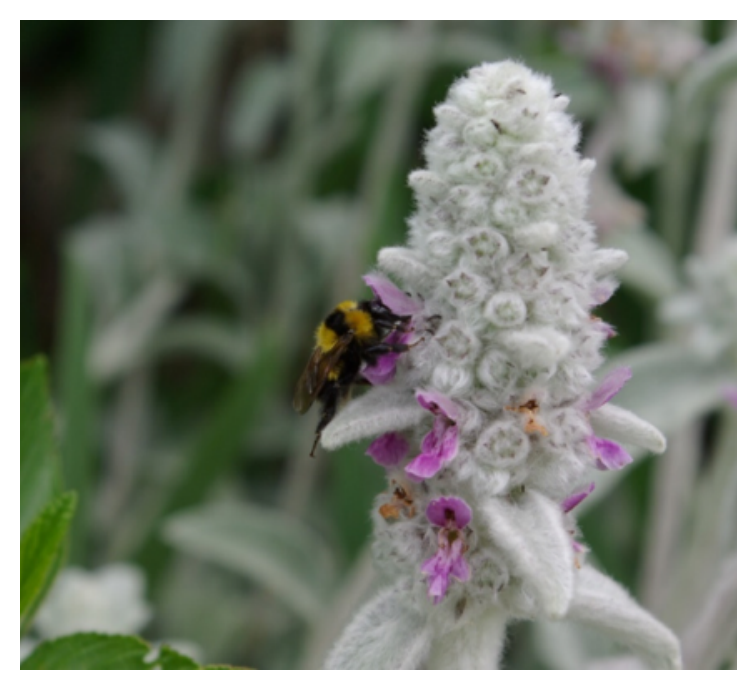

Figure 9. Mâle de B. argillaceus sur Stachys sp. (photo : B. Frin)

\section{Remarques}

Une seule ouvrière a été observée au cours de la première décade d'août (obs. pers. David 
Genoud). Deux données : une à Espinasses (18.VII.2012, Bernard Dardenne) et une à Embrun (24.VIII.2010 B. Frin), concernent des mâles collectés et identifiés car ils peuvent être confondus avec $B$. hortorum (figure 9). Une jeune reine a été observée pendant la troisième décade d'août 2014.

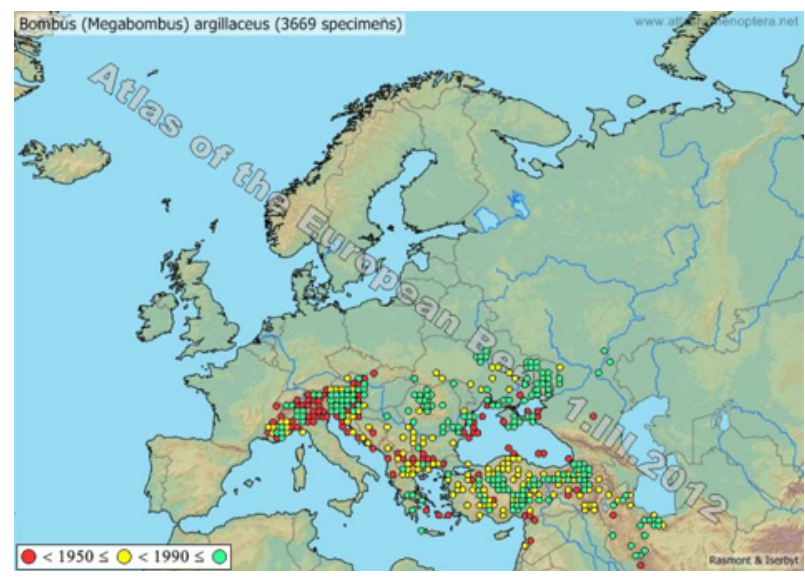

Figure 10. Bombus argillaceus en Europe et Asie.

\section{Discussion}

Le département des Hautes-Alpes est très riche par sa biodiversité et les abeilles ne font pas exception avec environ 300 espèces recensées fin 2016 (données non publiées). Parmi les espèces de Bombus qui fréquentent le département, on peut noter : $B$. gerstaeckeri, B. mendax, $B$. brodmannicus, $B$. sicheli, entre autres. $B$. argillaceus atteint dans les vallées alpines sa limite ouest de répartition (figure 10) et sa distribution mériterait d'être surveillée dans le cadre des changements climatiques en cours. De plus, l'espèce reste à rechercher dans le Briançonnais, le Queyras et le Dévoluy. Enfin nous pouvons nous poser la question du faible nombre d'ouvrière détectées ce qui peut venir du fait de la difficulté à les reconnaître avec certitude. De plus, la petite taille des colonies est aussi une des causes probables de cette faible détection. Enfin la dernière explication vient de notre protocole puisque celui-ci était ciblé sur les reines. Dans le futur nous pourront focaliser nos recherches sur les ouvrières principalement pendant la période estivale en juillet et août.

\section{Remerciements}

Mes remerciements s'adressent à tous les observateurs (Allombert Sylvain, Bernard Pierre, Boutin Michel, Braud Yoan, Brugot Didier, Classen Raphaël, Coulon Mireille, Dardenne
Bernard, Drouet Eric, Dusoulier François, Emmery Brigitte, Filosa Anne, Gagnaire Gilles, Genoud David, Maillot Roger, Paquet Isabelle, Raillot Jean, Rolland Blandine) et plus particulièrement à $\mathrm{F}$. Dusoulier (conservateur du Muséum d'histoire naturelle de Toulon et du Var) qui a bien voulu relire et corriger cette enquête et à Gilles Mahé pour le contrôle des déterminations des espèces de Bombus ainsi qu'aux membres de l'OA qui m'ont encouragé pour cette étude.

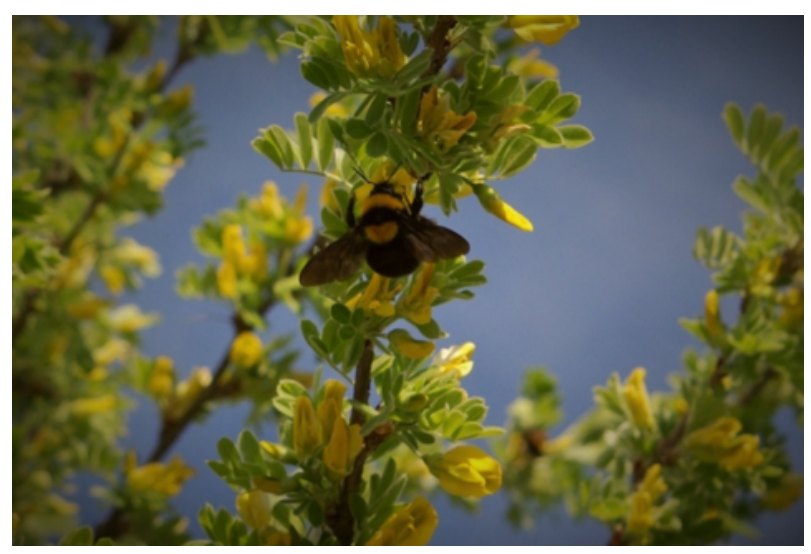

Figure II. Bombus argillaceus sur Caragana arborescens (Fabaceae). (Photo : B. Frin)

\section{Bibliographie}

EDWARdS M, Jenner M, 2018. Field guide of the Bumblebees of Great Britain and Ireland. Countryside and Garden Conservation Series. Ocelli. 108 p.

Chas E., 1994. Atlas de la flore des HautesAlpes. Conservatoire botanique national de GapCharance, Conservatoire des espaces naturels de Provence et des Alpes du sud, Parc National des Ecrins. 816 p.

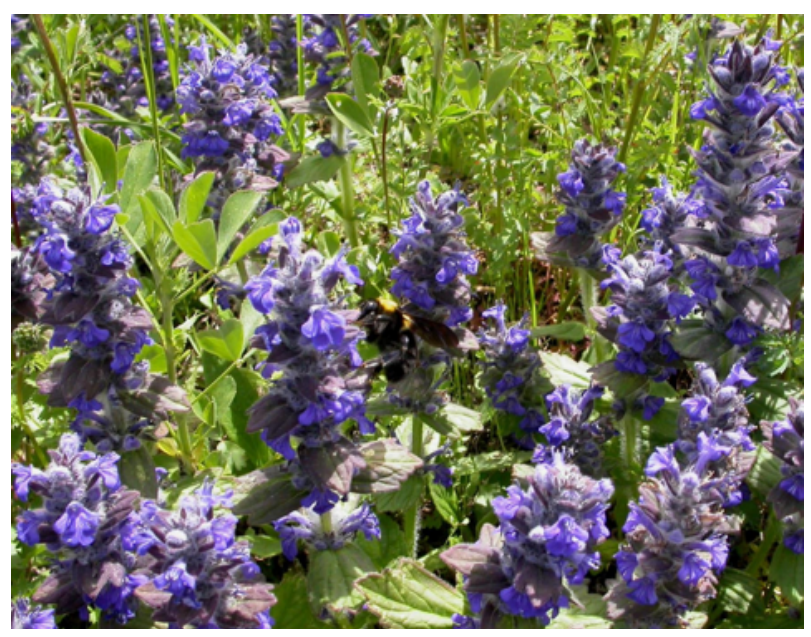

Figure 12 Bombus argillaceus sur Adjuga sp. (Lamiaceae). (Photo : B. Frin) 
Pouvreau A., 2004. Les Insectes Pollinisateurs La Bibliothèque du Naturaliste. Delachaux et Niestlè. 189 p.

RASMONT P, 1988. Monographie écologique et zoogéographique des Bourdons de France et de Belgique. Thèse de doctorat en Sciences Agronomiques, Faculté des Sciences agronomiques de l'Etat, Gembloux, 310 + LXII pp.

MAнÉ G, 2007. Observations de Bombus gerstaeckeri Morawitz (Hymenoptera, Apidae) butinant Delphinium dubium (Rouy et Fouc.) (Ranunculaceae) dans le Massif des Ecrins (Hautes-Alpes, France). Osmia 1 : 16-18.

MAHÉ G, 2008. Bourdons rares du Parc Naturel Régional du Queyras (Hautes-Alpes,France). Osmia $2: 21-25$.

MAHÉ G, 2011. Mémo pour l'atlas des bourdons armoricains. $\quad 11 \mathrm{p} . \quad \mathrm{http}: / /$ www.atlashymenoptera.net/biblio/ Memo2012_Atlas_Bombus_Armor.pdf

RASMONT P \& ISERBYT S, 2010-2013. Atlas of the European Bees: genus Bombus. 3d Edition. STEP Project, Atlas Hymenoptera, Mons, Gembloux. http://www.atlashymenoptera.net/ page.asp?ID=169 


\section{Écologie et distribution de l'abeille originaire d'Asie Megachile sculpturalis SMITH I 853 (Apoidea - Megachilidae - Megachilini) : un état des connaissances dix ans après sa première observation en Europe}

parViolette LE FÉON* et Benoît GESLIN***

Résumé. Megachile sculpturalis est une abeille originaire de l'est de l'Asie observée pour la première fois en Europe en 2008 à Allauch près de Marseille (France). Elle a également été détectée aux ÉtatsUnis dès 1994 et s'est depuis largement répandue en Amérique du Nord. Nous présentons ici un état des connaissances sur son écologie et sa distribution géographique.

Abstract. Megachile sculpturalis (the Giant Resin Bee) is a bee species native to East Asia that has been observed for the first time in Europe in 2008 in Allauch near Marseille (France). In the USA, it has been reported in 1994 and has since then rapidly expanded through North America. We here provide a review on its ecology and its geographical distribution.

Mots-clés : Megachile sculpturalis, distribution, espèce exotique, préférences alimentaires, nidification

Keywords : Megachile sculpturalis, distribution, exotic species, diet preferences, nesting behaviour

\section{Introduction}

Sur le continent américain et en Océanie, de nombreuses espèces d'abeilles exotiques (souvent originaires d'Europe) ont été observées (GOULSON 2003 ; RUSSO 2016). Leur présence hors de leur aire d'origine résulte d'introductions volontaires (par exemple Bombus terrestris (L. 1758) et Megachile rotundata (FABRICIUS 1793), espèces introduites pour la pollinisation et Apis mellifera L. 1758, introduite pour la pollinisation et la production de miel) ou involontaires. Dans le nord-est des États-Unis par exemple, BARTOMEUs et al. (2013) ont recensé 20 espèces

\footnotetext{
* Observatoire des Abeilles, 2 domaine Bellevue, I I 290 Arzens, France - violette.lefeon@gmail.com

** Aix Marseille Univ, Univ Avignon, CNRS, IRD, IMBE, AixMarseille Université - Campus Étoile Faculté des Sciences StJérôme Case 421 av Escadrille Normandie Niémen 13397 Marseille Cedex 20, France - benoit.geslin@gmail.com
}

d'abeilles exotiques et leur nombre ne cesse de croître (GIBBS \& DATHE 2017).

En Europe, les abeilles d'origine exotique sont plus rares. En 1996, une espèce nord-américaine, Xylocopa virginica (L. 1771), a été observée à quelques reprises en Angleterre (FALK \& LEWINGTON 2015). Dans leur récente mise à jour de la liste des abeilles d'Europe, RASMONT et al. (2017) citent plusieurs espèces du genre Xylocopa originaires d'Afrique ou du Proche-Orient récemment observées en Europe mais excepté pour $X$. pubescens SPINOLA 1838, bien établie en Grèce, les observations correspondantes ne semblent que ponctuelles. Enfin, Megachile disjunctiformis COCKERELL 1911, originaire de l'est de l'Asie, a été ponctuellement observée (5 spécimens au total) dans l'agglomération de Bologne en Italie en 2011, 2016 et 2017 (BorTolotTI et al. 2018). 
Megachile (Callomegachile) sculpturalis SMITH 1853, également originaire de l'est de l'Asie, est la seule espèce d'abeille introduite connue pour s'être installée dans plusieurs pays d'Europe. La première observation a été réalisée en 2008 dans le sud de la France (Vereecken \& Barbier 2009). Nous présentons ici un état des connaissances sur la distribution géographique et l'écologie de M. sculpturalis.

\section{Description}

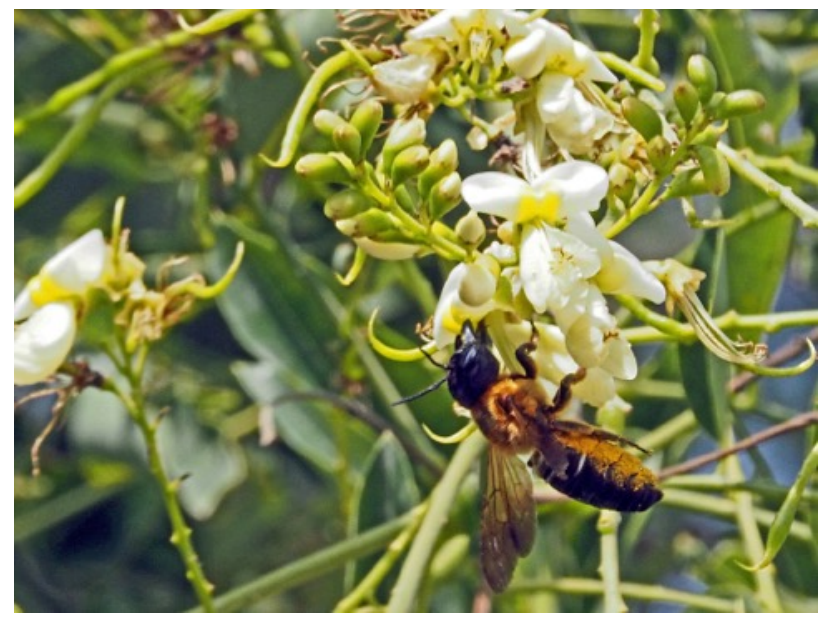

Figure I. Femelle de Megachile sculpturalis sur une fleur de Sophora japonica.

La brosse ventrale est recouverte de pollen.

Danièle Tixier-Inrep, Bouillargues (Gard), France, 2014.

M. sculpturalis est une mégachile de grande taille, les femelles mesurant de 22 à $27 \mathrm{~mm}$ de long et les mâles entre 14 et $19 \mathrm{~mm}$ (BATRA 1998). En France, rares sont les espèces dépassant deux centimètres de long : Xylocopa violacea (L. 1758), X. valga GERSTÄCKER 1872 et les reines de certains bourdons. Les individus des deux sexes ont des ailes fumées, un thorax recouvert de poils roux et un abdomen noir. Les femelles possèdent une brosse de poils (ou scopa) sous l'abdomen pour la récolte du pollen (fig. 1). Outre l'absence de brosse ventrale, les mâles se reconnaissent à la frange de poils clairs sur le clypéus, formant une sorte de moustache (fig. 2).

M. sculpturalis est facile à observer de par sa grande taille et son comportement (notamment car elle utilise fréquemment les hôtels à abeilles). Sous réserve de quelques précautions de rigueur (d'autres abeilles, des andrènes notamment, ont aussi un thorax roux et un abdomen noir), ses caractéristiques morphologiques en font une espèce facilement identifiable sur le terrain ou à partir d'une photographie. Elle ne peut normalement être confondue avec d'autres espèces d'abeilles en Europe, y compris avec l'abeille asiatique observée en Italie $M$. disjunctiformis qui est noire et blanche (BoRTOLOTTI et al. 2018). Le suivi de sa distribution géographique par un large réseau d'observateurs (sur le modèle des sciences participatives), même débutant dans l'étude des abeilles sauvages, est donc possible.

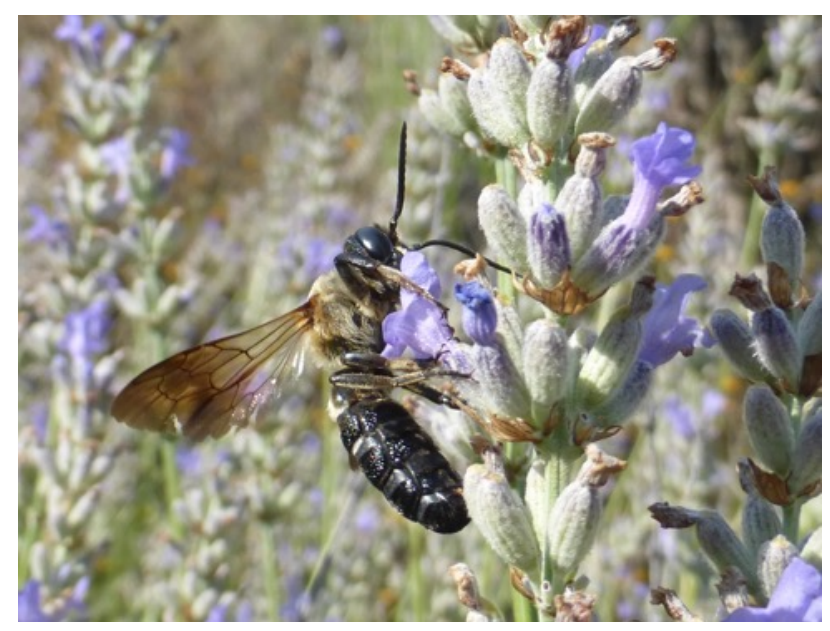

Figure 2. Mâle de Megachile sculpturalis consommant du nectar sur une fleur de lavande (Lavandula sp.).

Gilles Roux, Saint-Antonin-sur-Bayon

(Bouches-du-Rhône), France, 2016.

\section{Distribution géographique}

L'aire d'origine de $M$. sculpturalis comprend plusieurs pays de l'est de l'Asie : la Chine, le Japon, Taïwan et la péninsule coréenne (Hinojosa-DíAZ et al. 2005). C'est par ailleurs désormais une espèce exotique en Amérique du Nord et en Europe. Elle a été observée aux ÉtatsUnis (État de Caroline du Nord) dès 1994 (MANGUM \& BROOK 1997) et s'est depuis largement répandue (MANGUM \& SUMNER 2003 ; HiNOJOSA-DÍAZ 2008 ; PARYS et al. 2015). Elle occupe aujourd'hui presque toute la moitié est du pays, la limite ouest de répartition étant atteinte dans le Texas et la limite sud en Floride (PARYs et al. 2015). Elle est également présente au Canada (PAIERO \& BUCK 2003).

En Europe, M. sculpturalis a été observée pour la première fois en 2008 en France métropolitaine, à Allauch (13) près de la ville portuaire de Marseille (VEREECKEN \& BARBIER 2009). Elle a ensuite été observée en Italie en 2009 (QUARANTA et al. 2014), en Suisse près de la frontière italo-suisse en 2010 (AMIET 2012) puis au nord des Alpes en 2015 (DiLliER 2016), en Allemagne en 2015 (WESTRICH et al. 2015), en 
Hongrie en 2015 (Kovács 2015) et en Autriche en 2017 (P. Westrich, com. pers.) (fig. 3). La chronologie des découvertes ne reflète cependant pas nécessairement la chronologie réelle de son expansion. Par ailleurs, en l'absence d'analyses génétiques, il n'est pas établi si ces différentes populations sont issues d'une introduction unique (et le cas échéant si celle-ci a eu lieu en France) ou s'il y en a eu plusieurs (voir ZAYED $e t$ al. 2007 pour un exemple de ce type d'étude chez les abeilles).

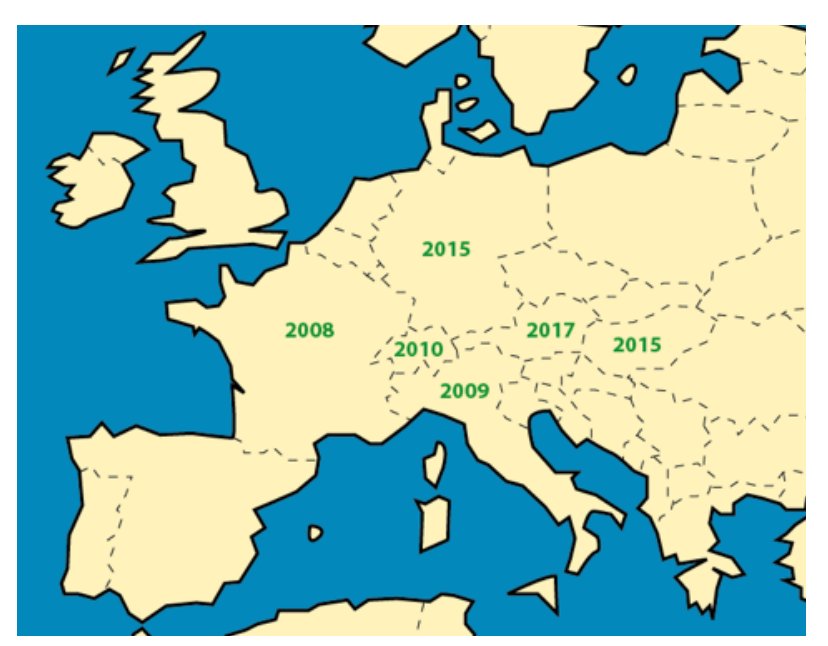

Figure 3. Année de première observation de Megachile sculpturalis dans les pays européens (Sources : Amiet 2012 ; Kovács 2015 ; Quaranta et al. 2014 ; Vereecken \& Barbier 2009 ; Westrich et al. 2015 ; Westrich, com. pers.).

En France, M. sculpturalis semble avoir rapidement étendu son aire de distribution depuis sa détection en 2008 (fig. 4). Entre 2008 et 2016, elle a été observée dans 72 communes, principalement dans le sud-est du pays, au sud de Lyon (69) et à l'est de Montpellier (34) (LE FÉON et al. 2018). Les observations ont eu lieu aussi bien au cœur de grandes villes que dans des zones rurales, et à une altitude maximale de 1540 m (un mâle observé à Matemale dans les Pyrénées-Orientales en 2013). En 2016, une nette progression vers l'ouest a été constatée (avec des observations à Toulouse (31), en Dordogne et dans les Pyrénées-Atlantiques à 520 $\mathrm{km}$ d'Allauch) et le nord (une femelle observée à Mâcon (71) à $335 \mathrm{~km}$ d'Allauch). En 2017², la présence de l'espèce dans l'ouest du pays se confirme avec une nouvelle observation aux environs de Toulouse (commune de Saint-Orens-
de-Gameville (31), 1 mâle, le 12 août 2017, par Hélène Laviron ${ }^{2}$ ) et dans le département des Landes (commune de Saint-Sever (40), 1 femelle, le 2 septembre 2017, par Philippe Dauge ${ }^{3}$ ) (fig. 4). En 2017, nous enregistrons également deux nouvelles observations en altitude, cette fois dans les Alpes-de-Haute-Provence (commune d'Allos (04), 1 mâle, le 2 août 2017, altitude = 1550 m, par Matthieu Aubert ; commune de Larche (04), 1 mâle, le 6 juillet 2017, altitude = 1846 m, par Eric Dufrêne) ainsi qu'une observation dans une région où l'espèce est déjà bien présente (commune de Saou (26), 1 individu, le 2 juillet 2017, par ascalaf074).

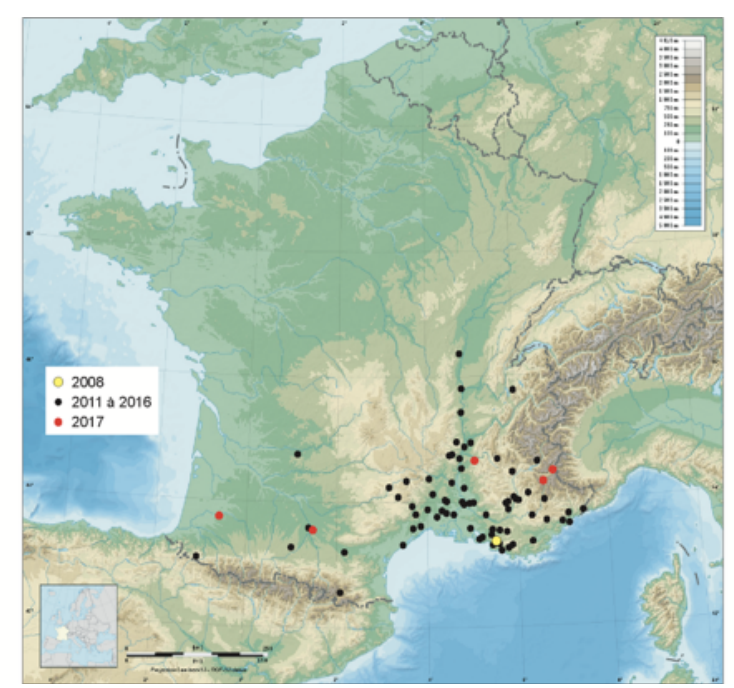

Figure 4. Distribution géographique de Megachile sculpturalis en France. Chaque point représente une commune. Le point jaune indique Allauch, lieu de la première observation de l'espèce en France, en 2008. Les points noirs indiquent les communes où une ou plusieurs observations ont eu lieu entre 2011 et 2016 (nous n'avons pas connaissance d'observations en 2009 et 2010). Les points rouges figurent les cinq communes mentionnées dans le texte, dans lesquelles la première observation a eu lieu en 2017.

Cette rapide expansion résulte probablement à la fois d'une composante naturelle (forte capacité de vol de l'espèce liée à sa grande taille) et d'une composante anthropique (déplacements d'adultes ou de larves par les échanges commerciaux, de bois notamment). Anthidium manicatum (L. 1758) (STRANGE et al. 2011) et Bombus terrestris (GESLIN \& MORALES 2015) sont deux autres exemples d'abeilles ayant rapidement étendu

\footnotetext{
I Nous mentionnons dans les lignes qui suivent les observations réalisées en 2017 constituant une première mention pour l'espèce dans la commune concernée.

${ }^{2}$ https://www.insecte.org/forum/viewtopic.php?f=3\&t=173263\&hilit=megachile+sculpturalis

3 https://www.flickr.com/photos/51044789@N02/369977I2745

${ }^{4}$ http://www.spipoll.org/galeries
} 
leur aire de distribution (respectivement en Amérique du Nord et en Patagonie) suite à une introduction hors de leur aire d'origine.

\section{Comportement alimentaire}

La nourriture exclusive des abeilles est constituée de ressources tirées des fleurs : le pollen (source de protéines), le nectar (source de sucres) et parfois des huiles florales (MICHENER 2007). Les adultes, mâles et femelles, consomment les ressources pour leurs besoins propres. De plus, les femelles récoltent les ressources (pollen principalement) pour les larves (exception faite des abeilles-coucous).

Le nombre de familles de plantes sur lequel une espèce d'abeille collecte du pollen détermine sa spécialisation alimentaire. Plusieurs auteurs ont proposé des classifications élaborées tenant compte de la diversité des comportements alimentaires (par exemple CANE \& SIPES 2006 ; Müller \& Kuhlmann 2008). Selon la classification simplifiée habituellement retenue, on qualifie d'oligolectique une espèce collectant le pollen sur des plantes d'une seule famille et de polylectique une espèce collectant le pollen sur des plantes de plusieurs familles (cf. par exemple NiETO et al. 2014). Le nectar est généralement collecté sur de nombreuses espèces végétales, y compris chez les abeilles oligolectiques.

Concernant le comportement alimentaire de $M$. sculpturalis dans son aire d'origine, BATRA (1998) indique qu'au Japon, le kudzu (Pueraria lobata = Pueraria hirsuta (THUNB.) C.K.SCHNEID. 1907, Fabacées) est sa source de pollen préférentielle, sans toutefois qu'elle ne soit exclusive, le même auteur citant plusieurs autres plantes. Pour la Chine, la plante citée est Lagerstroemia indica L. 1759, un arbuste de la famille des Lythracées.

Les observations réalisées aux États-Unis et en Europe indiquent que $M$. sculpturalis visite de nombreuses espèces végétales, de différentes familles, pour s'alimenter. Ainsi, dans leur synthèse de la littérature, PARYs et al. (2015) listent plus de 40 espèces végétales visitées, pour 21 familles. En France, entre 2008 et 2016, M. sculpturalis a été observée sur 20 espèces végétales appartenant à 8 familles différentes. 6 d'entre elles sont originaires d'Asie et ont été introduites comme plantes ornementales. Les autres sont des plantes indigènes (LE FÉON et al.
2018). Les plantes les plus fréquemment visitées étaient les lavandes (Lavandula spp., famille des Lamiacées) et l'arbre ornemental originaire d'Asie, Sophora japonica L. 1767 (famille des Fabacées). Les lavandes étaient la plante la plus visitée par les mâles, ce qui indique que c'est, au moins, une source de nectar préférentielle. Sophora japonica était la plante la plus visitée par les femelles.

Les observations de visites florales à elles seules ne permettent pas de statuer sur la spécialisation alimentaire d'une espèce d'abeille. L'analyse du contenu pollinique des scopa des femelles ou des cellules larvaires est nécessaire pour connaitre les espèces sur lesquelles le pollen est collecté et donc le statut de l'espèce, oligolectique ou polylectique. A notre connaissance, seuls sont disponibles les résultats d'analyses polliniques de quelques échantillons européens. En Italie, QUARANTA et al. (2014) ont trouvé que le contenu d'une cellule larvaire était composé de $81 \%$ de pollen de troène (genre Ligustrum, famille des Oléacées) et de $12 \%$ de pollen de châtaigner (genre Castanea, famille des Fagacées), d'autres pollens étant présents à l'état de traces. En Europe, les genres Ligustrum et Castanea comprennent une espèce indigène (respectivement L. vulgare L. 1753 et C. sativa Mill. 1768) et plusieurs espèces ornementales, notamment originaires d'Asie. Les résultats de QUARANTA et al. (2014) ne permettent pas de déterminer la nature du pollen au-delà du niveau générique et de conclure quant à l'origine européenne ou introduite des plantes utilisées.

WESTRICH et al. (2015) mentionnent le cas d'une cellule larvaire trouvée en Allemagne et de plusieurs cellules larvaires collectées en France ne contenant que du pollen de Sophora japonica. ANDRIEU-PONEL et al. (2018) ont analysé le pollen collecté sur plusieurs femelles à Nîmes et ont trouvé exclusivement du pollen de Sophora. Par ailleurs, le pollen d'une cellule larvaire collectée dans un hôtel à insectes à Montpellier était constitué de $97 \%$ de pollen de Sophora, les $3 \%$ restant provenant d'une trentaine d'espèces différentes (ANDRIEU-PONEL et al. 2018). Ces résultats posent la question des pollens présents en très petites quantités : proviennent-ils des récoltes de la femelle ou de résidus présents avant son installation dans cette cavité ?

Les analyses polliniques indiquent que $M$. sculpturalis collecte du pollen sur des plantes de 
différentes familles et peut donc être qualifiée de polylectique. Ces analyses sont cependant trop peu nombreuses pour conclure quant au niveau d'affinité de l'espèce avec certaines plantes et en particulier si elle dépend de la présence de plantes d'origine asiatique. L'espèce semble montrer une préférence pour $S$. japonica ou pour le genre Ligustrum mais dans quelles mesures ces résultats sont-ils généralisables ? C'est une question importante car l'approfondissement des connaissances permettra de déterminer si l'expansion de $M$. sculpturalis peut être limitée en l'absence de ces plantes nourricières et, parallèlement, si l'utilisation croissante de certaines plantes ornementales, telle que $S$. japonica, pourrait la favoriser.

\section{Nidification}

Comme de nombreuses espèces de Megachilidae, M. sculpturalis utilise des cavités préexistantes pour nidifier. Autrement dit, elle ne creuse pas elle-même, contrairement aux femelles de xylocopes par exemple, qui sont capables de creuser une cavité dans le bois. M. sculpturalis utilise divers types de cavités : " tiges " de bambou ou de canne de Provence, trous dans le bois mort (bûches) ou les vieux arbres, trous dans les poutres des maisons. Les cellules larvaires sont construites en résine de conifères ou d'érable par exemple (BATRA 1998), d'où son nom anglais de " Giant Resin Bee ». Les parois du nid, autour des cellules en résine, comportent de petites quantités de terre. Le bouchon terminal (qui sépare le nid de l'extérieur) est constitué de terre et parfois garni de débris divers, tels que des petits morceaux de bois, de paille, de coton (BATRA 1998) ou des pétales de fleurs (fig. 5). Sur les 39 cas de nidification recensés en France entre 2008 et 2016 (LE FÉON et al. 2018), les deux tiers concernent un hôtel à abeilles, avec dans certains cas l'emploi de tiges, dans d'autres de bûches percées. Pour le reste, la nidification a eu lieu dans des vieux arbres ou dans une poutre. Ce constat est peut-être un artefact associé à la plus forte présence d'observateurs aux abords des hôtels à abeilles (en général situés dans des lieux de passage : jardins, parcs) plutôt qu'autour des vieux arbres mais ce résultat témoigne d'une utilisation fréquente des hôtels à abeilles. Dans une étude initiée dans les parcs et jardins de la ville de Marseille, il est apparu que $M$. sculpturalis utilise massivement les hôtels à abeilles dès leur première année d'installation (B. Geslin, obs. pers.). Lors d'une étude menée sur trois ans dans la ville canadienne de Toronto, MACIVOR \& PACKER (2015) ont trouvé que les spécimens d'abeilles exotiques (parmi lesquels M. sculpturalis) représentaient $47,1 \%$ des abeilles collectées dans les hôtels à abeilles.

Dès les premières observations de $M$. sculpturalis aux États-Unis, l'utilisation de cavités précédemment utilisées par des xylocopes (X. virginica) a été constatée (MANGUM \& BROOK 1997) mais on ignorait si les femelles étaient venues occuper ces cavités une fois les occupants initiaux partis ou si celles-ci pouvait chasser les occupants pour s'installer dans leurs nids. Quelques années plus tard, LAPORT \& MINCKLEY (2012) ainsi que Roulston \& MALFI (2012) ont observé des femelles de $M$. sculpturalis chassant de leur nid des femelles de $X$. virginica puis vidant le contenu de la cavité afin d'y installer leurs propres cellules larvaires. Ce type de comportement avait déjà été observé chez une espèce européenne introduite aux États-Unis, Megachile apicalis SPINOLA 1808 (BARTHELL \& THORP 1995 in BARTHELL et al. 1998).

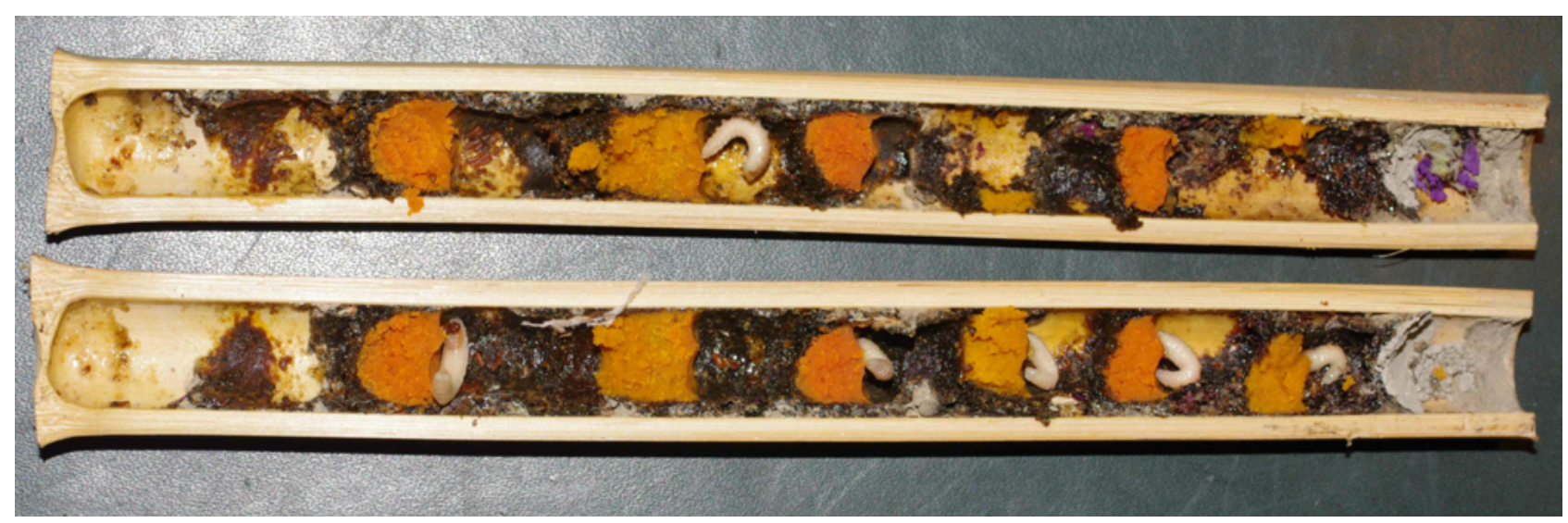

Figure 5. Tubes de bambous sectionnés pour observer la nidification de Megachile sculpturalis. Plusieurs cellules larvaires sont visibles, contenant une réserve de pollen et, parfois, une larve. Les cellules sont séparées les unes des autres par de la résine. Le bouchon terminal est constitué de terre et de pétales de fleurs (dans le bambou du haut). Daniel Mathieu,Avignon (Vaucluse), France, 2014. 


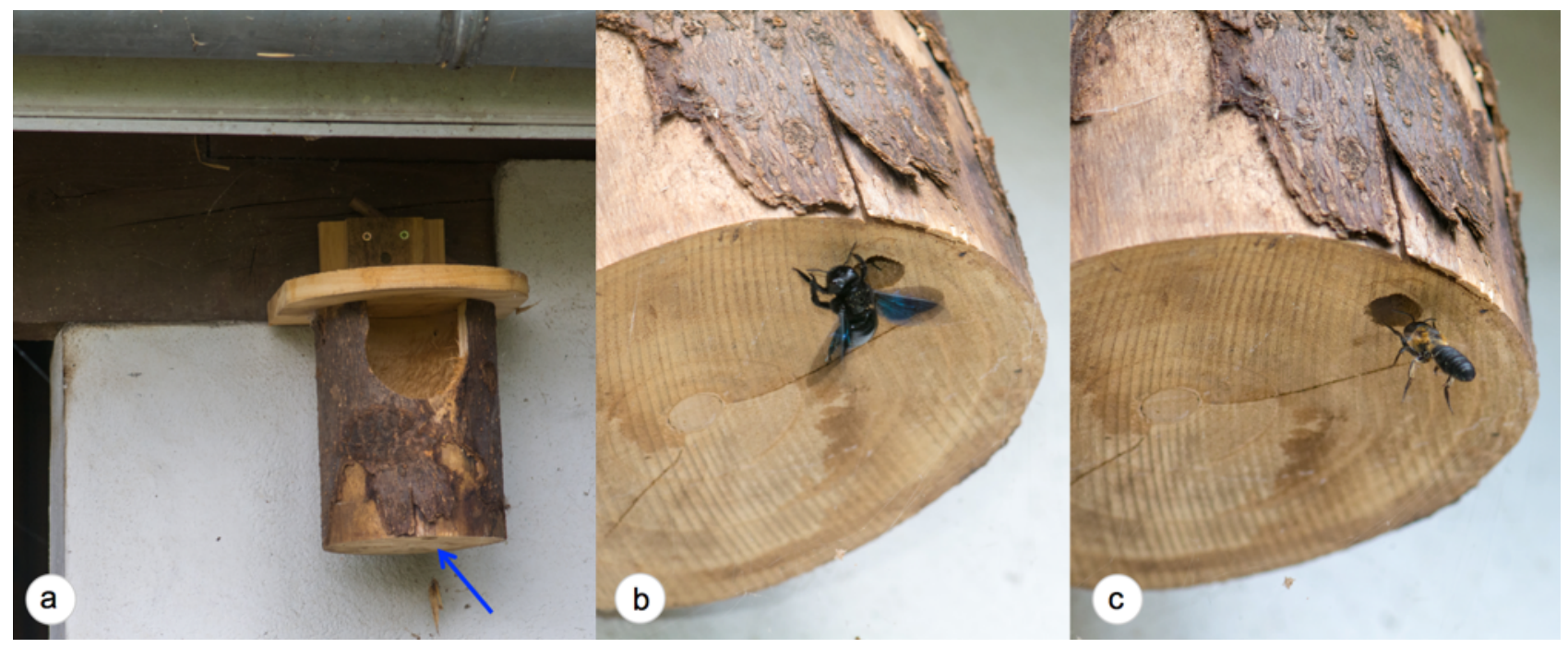

Figure 6. Trou dans une bûche (flèche bleue sur l'image a) dans lequel une femelle de Xylocopa violacea (b) et une femelle de Megachile sculpturalis (c) font des allers et venues au cours de la même journée.

Romain Baghi, Toulouse (Haute-Garonne), France, 2016.

En France, l'utilisation de cavités, dans du bois, précédemment occupées (et souvent creusées) par des xylocopes a été observée dans 5 communes différentes, selon les témoignages que nous avons recueillis. Des femelles de $X$. violacea et $M$. sculpturalis visitant la même cavité au cours de la même journée ont été observées (fig. 6) mais sans qu'un comportement agressif ou qu'un " vol » de nid (retrait du contenu d'un nid par M. sculpturalis pour y installer le sien) n'ait été constaté. Une cohabitation serait-elle possible au sein de mêmes cavités à l'image des cohabitations entre espèces du genre Xylocopa et Lithurgus (DUFRÊNE et al. 2016) ? Par contre, dans deux communes du sud de la France, des femelles de $M$. sculpturalis ont été observées alors qu'elles vidaient le contenu de cellules larvaires d'osmies (Osmia bicornis (L. 1758) et $O$. cornuta (LATREILLE 1805)) pour installer leurs propres nids. De la même façon, en Autriche, une femelle a été observée vidant le contenu du nid de la guêpe (elle-même exotique) Isodontia mexicana (SAUsSuRe, 1867) (fig. 7).

\section{Impacts écologiques}

Les abeilles véhiculent une image positive associée à l'apiculture et à leur rôle dans la pollinisation, largement médiatisé ces dernières années. Aussi, en comparaison d'autres animaux ou végétaux, une moindre attention est portée aux potentiels impacts négatifs sur les écosystèmes de l'introduction d'abeilles exotiques. Pourtant, plusieurs exemples montrent que ces introductions peuvent avoir des conséquences écologiques graves. Par exemple,
Bombus dahlbomii GUERIN-MeneviLle 1835, seule espèce de bourdon naturellement présente, et endémique, en Patagonie a rapidement décliné suite à l'introduction de deux espèces de bourdons européens, Bombus ruderatus (FABRICIUS 1775) et B. terrestris (MORALES et al. 2013).

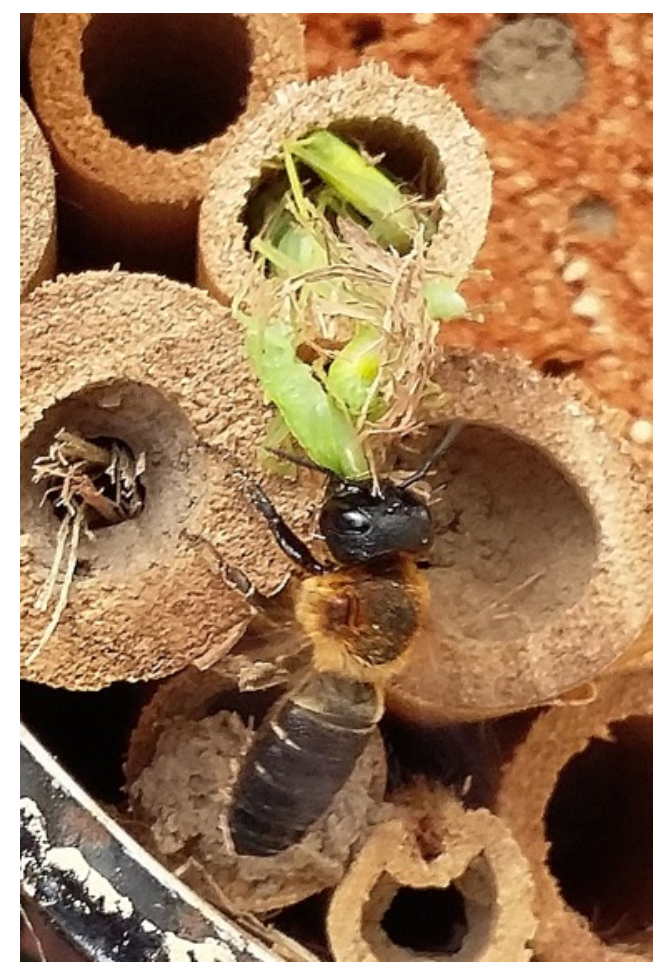

Figure 7. Femelle de Megachile sculpturalis en train de retirer d'une tige de bambou d'un hôtel à abeilles les larves d'orthoptères apportées pour sa progéniture par une femelle de la guêpe exotique Isodontia mexicana. Christian Treitner,Vienne, Autriche, 2017. 
GOULSON (2003) liste cinq types d'impacts négatifs associés à l'introduction d'abeilles exotiques : (1) la compétition pour les ressources florales avec les pollinisateurs indigènes ; (2) la compétition pour les sites de nidification ; (3) l'introduction conjointe de pathogènes ; (4) la pollinisation de plantes exotiques et (5) les effets négatifs sur la reproduction des plantes indigènes.

Nous manquons de recul aujourd'hui pour évaluer les impacts associés à la présence de $M$. sculpturalis hors de son aire d'origine. Les premières observations de terrain, en Amérique $\mathrm{du}$ Nord et en Europe, incitent cependant à porter une attention particulière à la compétition possible pour les sites de nidification entre $M$. sculpturalis et les espèces d'abeilles indigènes.

Si certains types de substrat sont en quantité limitée dans certains environnements, un phénomène de compétition peut subvenir. Par exemple, l'occupation par M. sculpturalis de cavités creusées par des xylocopes peut-elle affecter à plus ou moins long terme les populations de ces derniers ? Par ailleurs, $M$. sculpturalis a un impact direct sur la mortalité d'autres insectes en vidant les nids et les larves qui s'y trouvent. Il est difficile d'estimer la fréquence de ce type d'évènements et ses conséquences pour les populations d'insectes concernés. En Europe, certaines espèces des genres Anthidium, Lithurgus, Osmia, Xylocopa ou encore Megachile lagopoda (L., 1761) (David Genoud, com. pers.) sont des espèces susceptibles d'être négativement affectées par la compétition pour les sites de nidification ou l'usurpation de nids, dans des situations de populations fragiles ou de sites de nidification en nombre limité.

\section{L'histoire continue}

A l'instar du Frelon asiatique (Vespa velutina LEPELETIER 1836), de la Coccinelle asiatique (Harmonia axyridis (PALLAS 1773)), de la Pyrale $\mathrm{du}$ buis (Cydalima perspectalis (WALKER 1859)), $\mathrm{du}$ Longicorne asiatique (Anoplophora glabripennis (MotschULSKY 1854)) ou du Moustique tigre (Aedes albopictus (SKUSE,1894)), M. sculpturalis a rejoint la longue liste d'insectes originaires d'Asie installés en France métropolitaine. Où en est sa présence en France? Dans les zones du pays qu'elle occupe désormais, elle est fréquemment signalée dans les espaces urbains, où de nombreuses personnes circulent et sont interpelées par cette grande abeille peu discrète. Est-elle autant présente dans les zones naturelles ? Quel est le niveau d'affinité avec Sophora japonica? Se maintiendrait-elle dans des espaces où Sophora japonica (et autres espèces végétales originaires d'Asie) seraient absentes (en particulier les zones naturelles) ? Nous encourageons les observateurs à prendre des photos et à noter le maximum de détails (photographies, date, lieu, comportement, plantes visitées, substrats utilisés pour la nidification, interactions avec d'autres abeilles). Cela permettra d'approfondir les connaissances sur l'écologie de $M$. sculpturalis et de mieux évaluer ses impacts sur la faune et la flore des pays d'introduction.

\section{Remerciements}

Nous remercions toutes les personnes qui ont partagé leurs observations de Megachile sculpturalis (voir LE FÉON et al. 2018 pour la liste complète), notamment sur les forums Apoidea-Gallica et Le Monde des insectes et sur le site du Spipoll. Merci à Valérie Andrieu-Ponel, Matthieu Aubert, Philippe Dauge, Mathieu de Flores, David Genoud, Gilles Roux et Paul Westrich pour leur aide dans la collecte d'informations sur la distribution et l'écologie de l'espèce. Merci à Romain Baghi, Daniel Mathieu, Gilles Roux, Danièle Tixier-Inrep et Christian Treitner pour avoir accepté l'utilisation de leur photo. Merci à Gérard Le Goff pour l'identification du xylocope de la figure 6. Merci à Matthieu Aubert et Lise Ropars pour leur relecture. Les auteurs des fonds de carte sont Arnaud Le Nevé (fig. 3) et Eric Gaba (fig. 4).

\section{Références bibliographiques}

Amiet F, 2012. Die Blattschneiderbiene Megachile sculpturalis Smith, 1853 (Hymenoptera, Apidae) nun auch in der Schweiz. Entomo Helvetica 5 : 157 - 159.

ANdrieu-Ponel V, Ponel P, Le FÉON V, Geslin B \& Duvallet G, 2018. À propos du comportement de butinage de Megachile sculpturalis Smith, 1853, en France méditerranéenne (Nîmes et Montpellier) (Hymenoptera, Megachilidae). Bulletin de la Société entomologique de France 123 : 49 - 54.

BARThell JF, Frankie GW \& Thorp RW, 1998. Invader effects in a community of cavity nesting megachilid bees (Hymenoptera: 
Megachilidae). Environmental Entomology 27 : $240-47$.

BARThell JF, ThORP RW, 1995. Nest usurpation among females of an introduced leafcutter bee, Megachile apicalis. Southwestern Entomologist $20: 117$ - 124 .

Bartomeus I, Ascher JS, Gibbs J, DANFORTH BN, WAGNER DL, HEDTKE SM \& WiNFREE R, 2013. Historical changes in northeastern US bee pollinators related to shared ecological traits. Proceedings of the National Academy of Sciences $110: 4656$ - 4660.

BATRA SWT, 1998. Biology of the Giant Resin Bee, Megachile sculpturalis Smith, a conspicuous new immigrant in Maryland. The Maryland Naturalist $42: 1-3$.

Bortolotti L, Luthi F, Flaminio S, Bogo G, SGolastra F, 2018. First record of the Asiatic bee Megachile disjunctiformis in Europe. Bulletin of Insectology $71: 143-149$.

Dillier F-X, 2016. Eingeschleppte Asiatische Mörtelbiene Megachile sculpturalis Smith,

1853 (Hymenoptera, Apidae) erstmals nördlich der Alpen gesichtet. Entomo Helvetica $9: 153-$ 156.

Dufrêne É, Genoud D \& Bourlet P, 2016. Sur la distribution en France de Lithurgus cornutus Fabricius, 1827 (Hymenoptera Megachilidae) : apport de données récentes. Osmia $6: 16-21$.

FALK S \& LeWINGTON R, 2015. Field guide of the bees of Great Britain and Ireland. British Wildlife Field Guides. Bloomsbury.

GeSLin B \& MoRALES CL, 2015. New records reveal rapid geographic expansion of Bombus terrestris Linnaeus, 1758 (Hymenoptera: Apidae), an invasive species in Argentina. Checklist $11: 1620$.

GIBBS J \& DATHE HH, 2017. First records of Hylaeus (Paraprosopis) pictipes Nylander, 1852 (Hymenoptera: Colletidae) in North America. Check List 13 : 2116.

Goulson D, 2003. Effects of introduced bees on native ecosystems. Annual Review of Ecology, Evolution and Systematics 34 : 1-26.

HinoJoza-Diaz IA, YAÑEZ-ORdoñez O, CheN G, Perterson AT \& Engel MS, 2005. The North American invasion of the giant resin bee (Hymenoptera: Megachilidae). Journal of Hymenoptera Research $14: 69-77$.

HinOJOSA-DIAZ IA, 2008. The giant resin bee making its way west: first record in Kansas (Hymenoptera: Megachilidae). ZooKeys 1: 67 71.
KovÁCs T, 2015. Megachile sculpturalis Smith, 1853 in Hungary (Hymenoptera, Megachilidae). Folia Historico-naturalia Musei Matraensis 39 : $73-76$.

LAPORT RG \& MINCKLEY RL, 2012. Occupation of active Xylocopa virginica nests by the recently invasive Megachile sculpturalis in Upstate New York. Journal of the Kansas Entomological Society $85: 384-386$.

Le FÉon V, Aubert M, Genoud D, AndrieuPonel V, Westrich P \& Geslin B, 2018. Range expansion of the Asian native giant resin bee Megachile sculpturalis (Hymenoptera, Apoidea, Megachilidae) in France. Ecology and Evolution 8: $1534-1542$.

MANGUM WA \& BRoOKS RW, 1997. First records of Megachile (Callomegachile) sculpturalis Smith (Hymenoptera: Megachilidae) in the continental United States. Journal of the Kansas Entomological Society 70 : 140 - 142.

MACIVOR JS \& PACkER L, 2015. 'Bee hotels' as tools in native pollinator conservation: a premature verdict? PLoS ONE 10 : e0122126.

Mangum WA, Sumner S, 2003. A survey of the North American range of Megachile (Callomegachile) sculpturalis, an adventive species in North America. Journal of the Kansas Entomological Society $76: 658-662$.

MICHENER CD, 2007. The bees of the world. 2nd ed. The Johns Hopkins University Press, Baltimore.

Morales CL, Arbetman MP, Cameron SA \& AIZEN MA, 2013. Rapid ecological replacement of a native bumble bee by invasive species. Frontiers in Ecology and the Environment 11 : $529-534$.

NiETO A, ROBERTS SPM, KEMP J, RASMONT P, Kuhlmann M, García Criado M, BiEsmeiJer JC, Bogusch P, DAthe HH, De La RÚa P, DE Meulemeester $T$, Dehon $M$, Dewulf $A$, Ortiz-Sánchez FJ, Lhomme P, Pauly A, Potts SG, Praz C, QUaranta M, RAdChenko VG, Scheuchl E, SMit J, Straka J, Terzo M, TomoziI B, Window J \& Michez D, 2014. European Red List of bees. Luxembourg, Publication Office of the European Union.

Quaranta M, Sommaruga A, Balzarini P \& FELICIOLI A, 2014. A new species for the bee fauna of Italy: Megachile sculpturalis continues its colonization of Europe. Bulletin of Insectology $67: 287-293$.

Parys K, Tripodi A, Sampson B, 2015. The Giant Resin Bee, Megachile sculpturalis Smith: new distributional records for the Mid- and Gulfsouth USA. Biodiversity Data Journal 3:e6733 
Rasmont P, Devalez J, Pauly A, Michez D \& RADChENKo VG, 2017. Addition to the checklist of IUCN European wild bees (Hymenoptera: Apoidea). Annales de la Société Entomologique de France (N.S.) $53: 17$ - 32.

ROUlston T \& MALFI R, 2012. Aggressive eviction of the Eastern Carpenter Bee (Xylocopa virginica (Linnaeus)) from its nest by the Giant Resin Bee (Megachile sculpturalis Smith). Journal of the Kansas Entomological Society 85 : $387-388$.

RUSSo L, 2016. Positive and negative impacts of non-native bee species around the world. Insects $7: 69$.

Strange JP, KOCH JB, Gonzalez VH, NemelKa L \& GRISWOLd T, 2011. Global invasion by Anthidium manicatum (Linnaeus) (Hymenoptera: Megachilidae): assessing potential distribution in North America and beyond. Biological Invasions 13 : 2115 - 2133.

VEREECKEN NJ \& BARBIER E, 2009. Premières données sur la présence de l'abeille asiatique Megachile (Callomegachile) sculpturalis Smith (Hymenoptera, Megachilidae) en Europe. Osmia $3: 4-6$.

WESTRICH P, KNAPP A \& BERNEY I, 2015. Megachile sculpturalis Smith 1853 (Hymenoptera, Apidae), a new species for the bee fauna of Germany, now north of the Alps. Eucera $9: 3-10$.

Zayed A, Constantin SA, Packer L, 2007. Successful biological invasion despite a severe genetic load. PLoS ONE 2 : e868. 


\section{Quelques notes de lecture}

par Tanguy JEAN*

\section{Quelques publications récentes d'Entomofauna}

Entomofauna, Zeitschrift für Entomologie, est une revue bien connue des entomologistes publiée par Thomas Witt et dont le rédacteur-enchef n'est autre que l'apidologue reconnu Maximilian Schwarz. Cette revue propose régulièrement des travaux sur les Abeilles. Récemment, trois publications d'importance ont attiré notre attention.

Entomofauna peut être commandée auprès de Maximilian Schwarz (maximilian.schwarz@liwest.at) ou de Thomas Witt (twittmuseum@gmail.com); il est même recommandé d'écrire à ces deux personnes en même temps, en allemand ou en anglais. Les articles sont généralement expédiés rapidement avec une facture pro-forma à acquitter par virement sur le compte bancaire de Th. Witt.

\section{Deux publications sur les Anthidiini}

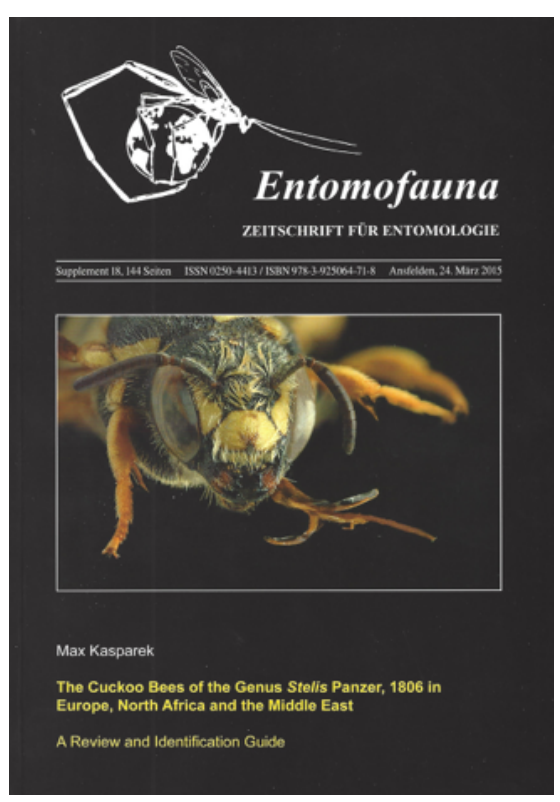

MAX KASPAREK, 2015. The Cuckoo Bees of the Genus Stelis Panzer, 1806 in Europe, North Africa and the Middle East. A Review and Identification Guide. Entomofauna, supplement 16: 1-144.

*Tanguy JEAN - Observatoire des Abeilles, 8 allée du Château, F - 59700 Marcq-en-Barœul, France - tanguy.jean4@gmail.com

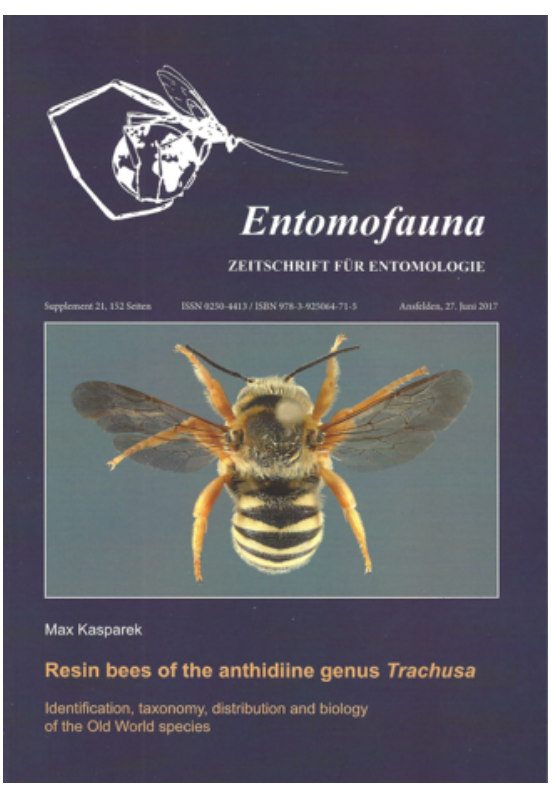

MAX KASPAREK, 2017. Resin bees of the Anthidiine genus Trachusa. Identification, taxonomy, distribution and biology of the Old World species. Entomofauna, supplement 21: 1-152.

Max Kasparek est un zoologiste allemand, plutôt vertébriste à l'origine et fortement tourné vers le Proche et le Moyen-Orient puisqu'il est rédacteur-en-chef de la revue Zoology in the Middle East. Son entrée dans le monde des publications entomologiques est fracassante puisqu'il a décidé de consacrer les années à venir à la révision de la tribu des Anthidiini dans la zone ouest-paléarctique (comm. pers. de l'auteur). Il reçoit les encouragements et l'aide de Maximilian Schwarz - qui est aussi caution scientifique sur ces genres qu'il maîtrise bien.

En 2015, Max Kasparek publie un premier travail de révision complète sur le genre Stelis de la zone ouest-paléarctique puis, en 2017, un second travail sur le genre Trachusa, cette fois-ci étendu à "l'Ancien Monde " puisque ce genre est très représenté en Afrique, les espèces estpaléarctiques voire orientales (au sens biogéographique) étant par ailleurs incluses, même si beaucoup reste sans doute à faire à leur sujet. Par rapport à d'autres travaux plus ou moins récents qui reprenaient des synthèses 
anciennes, il s'agit ici d'un travail en grande partie original se basant aussi bien sur la littérature passée que sur une révision approfondie des taxons, avec l'élucidation de diverses difficultés taxonomiques.

Chaque ouvrage comprend une partie introductive très détaillée, des clefs de détermination des sous-genres et des espèces et des monographies d'espèces relativement exhaustives. Ces monographies incluent la synonymie, des descriptions précises des deux sexes et ce qu'on sait de la biologie des espèces et de leur répartition, avec des cartes montrant les pays d'où l'espèce est connue. L'ensemble est très richement illustré de clichés originaux abondants et de nombreuses figures au trait, aussi bien extraites de travaux antérieurs qu'originales, qui rendent aisé l'usage de ce travail très complet. Des tableaux comparatifs très utiles sont par ailleurs proposés pour les clusters d'espèces proches. L'ensemble est édité en couleurs, sous forme de revues brochées.

Devant un travail de cette qualité exceptionnelle, on attend avec une impatience manifeste et difficile à cacher la suite des publications de l'auteur sur les autres genres de la tribu des Anthidiini !

\section{Une publication sur les Nomada européennes}

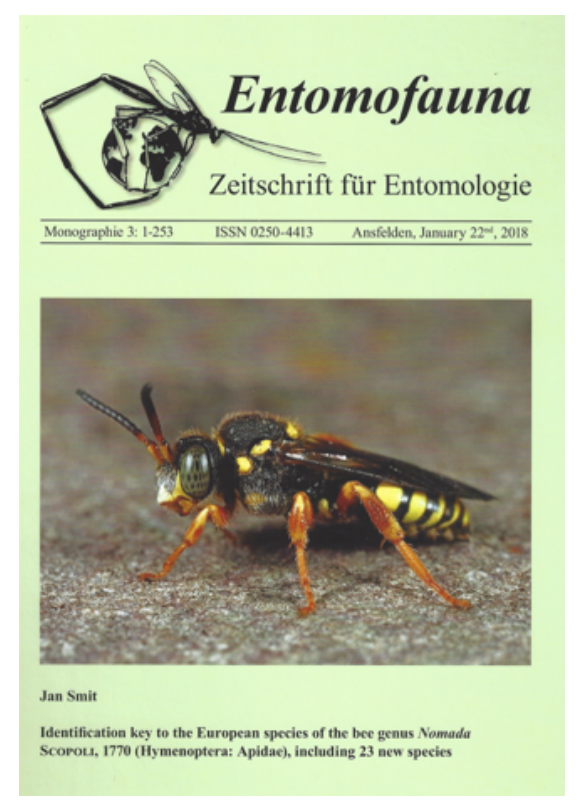

JAN SMIT, 2018. Identification key to the European species of the bee genus Nomada Scopoli, 1770 (Hymenoptera: Apidae), including 23 new species. Entomofauna, Monographie 3: 1-253.
Le genre Nomada est une des bêtes noires des apidologues et les personnes capables de les identifier sérieusement, notamment si l'on se réfère aux espèces méditerranéennes qui manquaient jusqu'ici cruellement d'une synthèse récente, se comptent peut-être sur les doigts d'une ou deux mains en Europe. Le Néerlandais Jan Smit s'intéresse à ce genre depuis plusieurs années et a bénéficié du compagnonnage de Maximilian Schwarz qui a passé sa vie à étudier les Nomada et est aussi co-auteur des descriptions d'espèces nouvelles dans cette toute nouvelle synthèse européenne.

Le travail réalisé dans cet ouvrage est monumental et objectivement remarquable : outre une clef originale (E. Dufrêne, comm. pers. sur la liste Apoidea-gallica) illustrée de nombreux clichés de détail très utiles, ce travail comprend en outre une courte partie introductive et des monographies d'espèces, où sont abordées la synonymie, la répartition (avec des cartes montrant les pays où chaque espèce est connue en Europe), la saison de vol et la ou les espèceshôtes quand elles sont connues et, bien sûr, la diagnose des deux sexes. La description de 23 espèces nouvelles est également à noter ; des clichés d'habitus et de détails complètent ces descriptions.

Ainsi, il est désormais possible à n'importe quel entomologiste d'avoir accès à tout ce qu'on sait à ce jour des 208 espèces européennes du genre, $\mathrm{y}$ compris les taxons méditerranéens ! Et d'essayer de les identifier, même si le travail reste évidemment corsé. Nul doute que ce travail très précieux est déjà une bible et est appelé à le rester pour plusieurs décennies! 


\section{Quelques livres}

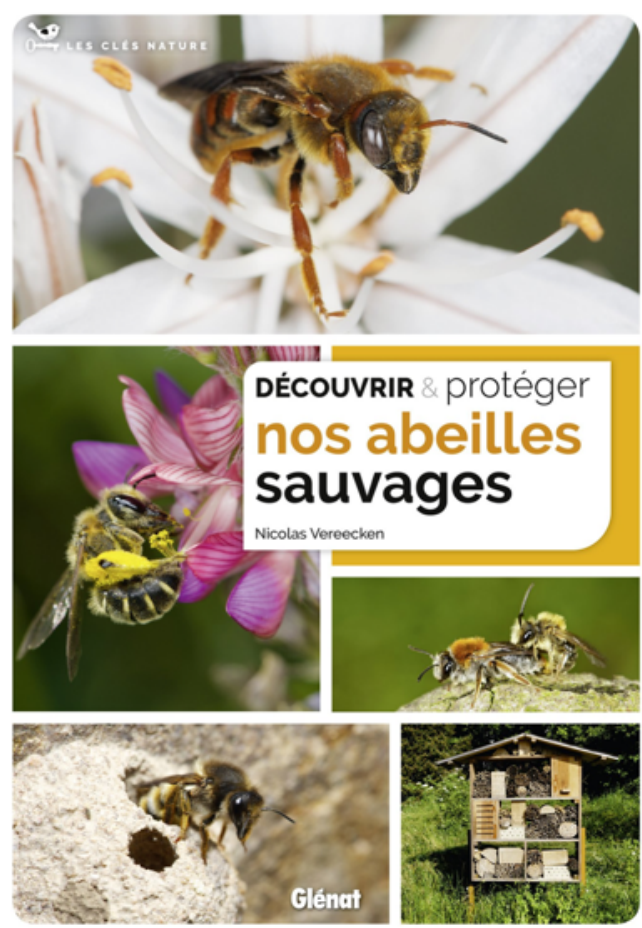

NiCOLAS VeREECKEN, 2017. Découvrir et protéger nos abeilles sauvages. Glénat, Grenoble, 191 pages.

Bien connu de la communauté apidologique francophone, Nicolas Vereecken est un entomologiste belge aujourd'hui professeur à l'Université libre de Bruxelles. Celui qui a fondé la revue Osmia s'intéresse aux Abeilles depuis de nombreuses années et a toujours montré un dynamisme entomologique impressionnant.

Dans ce travail richement illustré de magnifiques photographies pour beaucoup dues à l'auteur, Nicolas ne nous déçoit pas et nous emmène dans un voyage initiatique passionnant au plus près de ces Insectes. L'ouvrage intéressera aussi bien les débutants qui pourront ainsi entrer dans le monde des Abeilles sauvages que les entomologistes plus chevronnés qui ne manqueront pas de découvrir ou redécouvrir des informations variées au sujet de ces Hyménoptères surprenants.

La première partie de l'ouvrage est dédiée à une présentation biologique et écologique des Abeilles. On y aborde ainsi leur origine et leur diversité, leur morphologie, leur cycle de vie, des aspects variés de leur comportement (mimétisme, comportements sociaux), leur cycle de vie, leur importance écologique et évolutive, leurs ennemis naturels ou encore les lieux où les chercher.

La deuxième partie de l'ouvrage se penche plus en détail sur la diversité du groupe en l'abordant d'un point de vue non pas systématique - peutêtre plus austère pour le débutant - mais plus original, à savoir éthologique, en abordant successivement les différents modes de vie de ces Insectes : Abeilles terricoles, caulicoles, " squatteuses " d'anfractuosités variées, maçonnes, cardeuses (cotonnières), coupeuses de feuilles et de fleurs, hélicicoles, charpentières, résinières et parasites.

Chère à l'auteur, la protection des Abeilles n'est pas en reste puisqu'elle est l'objet de la troisième partie. Nicolas évoque ainsi le déclin de ces Insectes mais aussi les initiatives qui peuvent contribuer à maintenir leur diversité. Un tableau des espèces végétales appréciées des Abeilles complète l'ouvrage, ainsi que des pistes bibliographiques et associatives pour se lancer dans leur étude.

Un livre à la passion communicative qui peut et doit - être mis en toutes les mains !

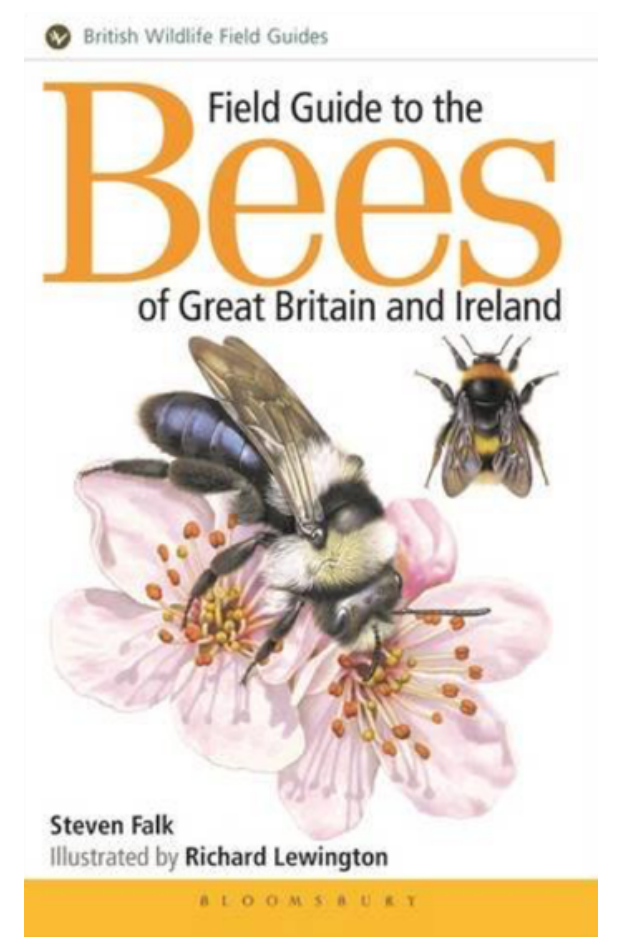

Steven FalK \& Richard LeWington, 2015. Field Guide to the Bees of Great Britain and Ireland. Blomsbury, London, 432 pages. 
Voilà un ouvrage de qualité comme les naturalistes anglais savent les faire. On ne regrettera qu'une chose : qu'il soit réduit aux espèces des Îles britanniques et qu'il n'en existe pas encore d'équivalent pour les espèces françaises.

Dans ce livre, les auteurs proposent une introduction très précise et bien pensée, des clefs de détermination avec des clichés de détails ou dessins au trait illustrant bon nombre de critères, et des monographies d'espèces très complètes. Ces dernières comprennent de courtes descriptions mais aussi des indications biologiques qui y tiennent la part majeure (saison de vol, fleurs visitées, habitudes de nidification, parasites/hôtes...). Des petites cartes de distribution les complètent. Il convient de préciser que chaque monographie comprend des clichés en couleur, généralement des deux sexes! Vingt planches originales de dessins en couleurs dues à $R$. Lewington complètent le tout.

Cet ouvrage trouvera sa place dans la bibliothèque de n’importe quel apidologue!

\section{Solitary bees}

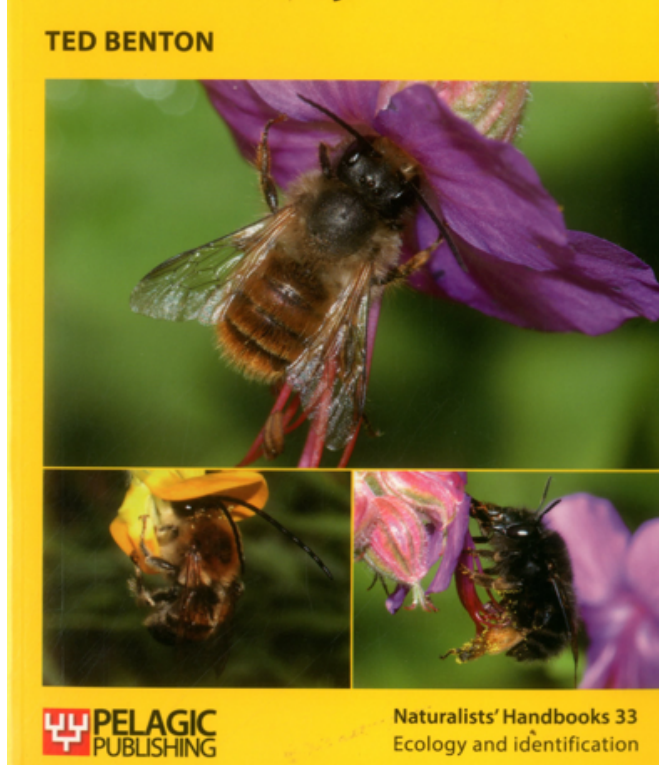

Ted Benton, 2017. Naturalists' Handbooks 33. Solitary bees. Pelagic Publishing, Exeter (UK), 202 pages.

Cet ouvrage naturaliste est avant tout une présentation biologique et écologique des
Abeilles. On y trouve successivement une introduction où la morphologie est abordée, un panorama de la diversité du groupe, une longue présentation des modes de vie des Abeilles, une partie sur les Abeilles coucous, une autre sur les liens entre fleurs et Abeilles, une avant-dernière sur la conservation de ces Insectes et enfin une clef de détermination des genres. Un livre intéressant que l'on ne peut que recommander.

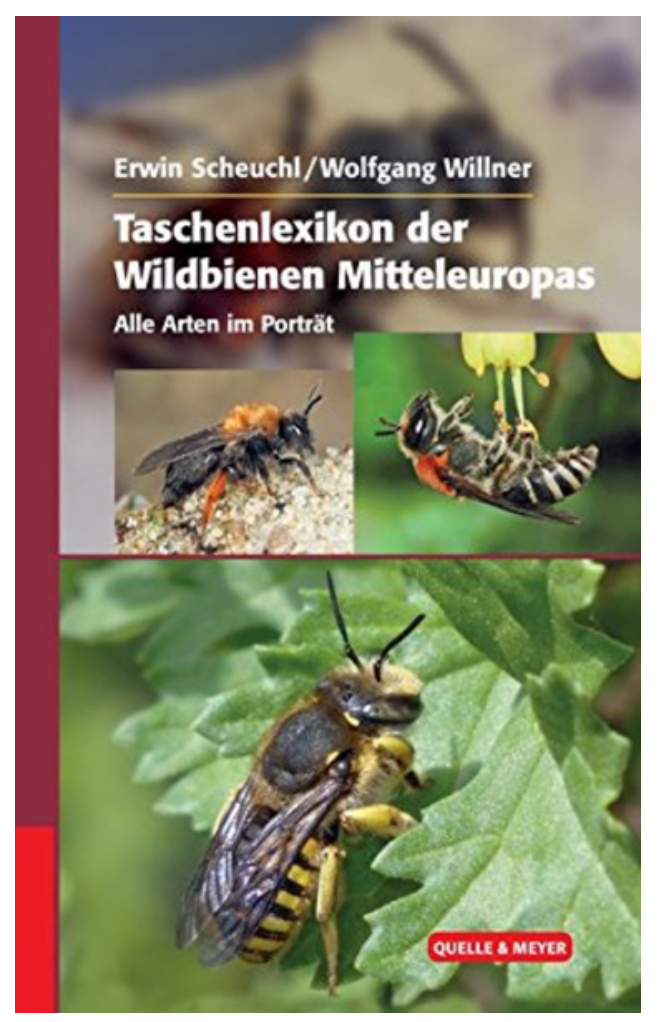

ErWin Scheuchl \& Wolfgang Willner, 2017. Taschenlexikon der Wildbienen Mitteleuropas. Quelle $\&$ Meyer, Wiebeksheim (G), 917 pages.

Rêveriez-vous d'un ouvrage qui décrit en détail la biologie et la répartition de chaque espèce d'Abeille de votre pays et dans votre langue ? Eh bien, cela n'existe pas encore pour nous. Mais les apidologues germanophones possèdent désormais ce gros pavé pour les espèces d'Europe moyenne. Après de courtes parties introductives, cet ouvrage propose des monographies (rangées par ordre alphabétique) de toutes les Abeilles de cette zone dont un certain nombre est enrichi de clichés photographiques. Attention, toutefois, il n'y a ni descriptions morphologiques, ni clefs de détermination ; ce n'est donc clairement pas un livre d'identification. Cet office est rempli par les clefs allemandes du premier auteur ou les faunes suisses. 


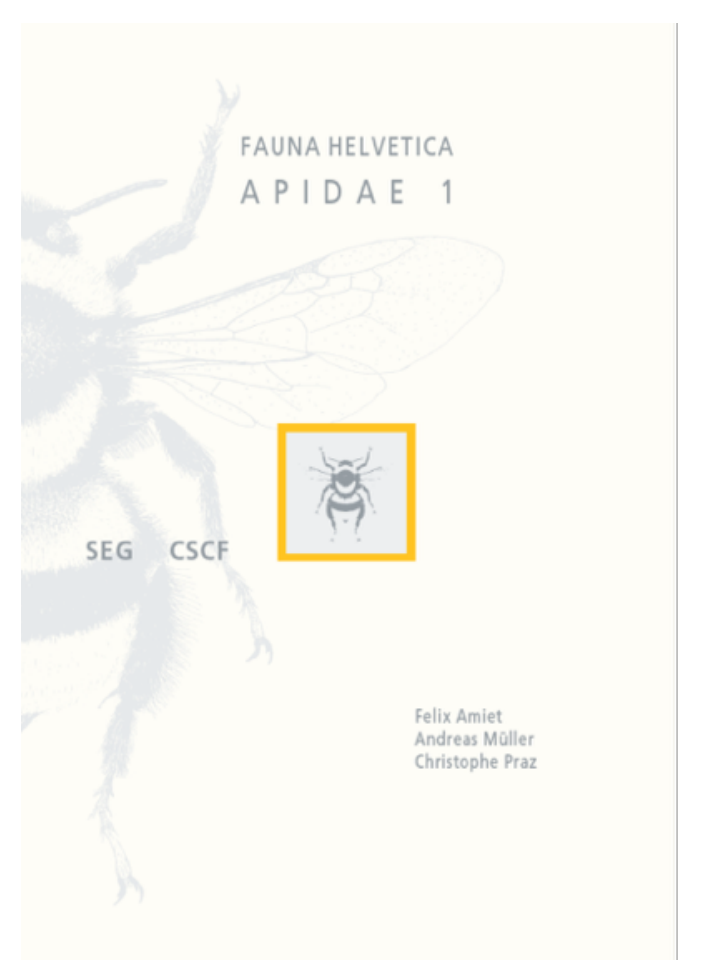

Felix Amiet, ANdreas Müller \& Christophe

Praz, 2017. Fauna helvetica. 29. Apidae 1. Partie générale. Genres. Bombus. Apis. CSCF-SEG, Neûchatel $(\mathrm{CH}), 188$ pages.

La série Fauna helvetica s'élargit avec une révision de l'ouvrage jadis publié par Felix Amiet au sujet des Bourdons, cette fois-ci enrichi d'une traduction française des clefs de détermination et d'une mise à jour des données cartographiques. Certains dessins ont été également refaits pour l'occasion. Cet ouvrage de très grande qualité, dans la lignée des précédents, saura nous rendre de fiers services dans nos identifications. 


\section{GALERIE PHOTOS}

Quelques exemples d'oligolectisme chez les abeilles sauvages. Par Géraud DE PRÉMOREL.

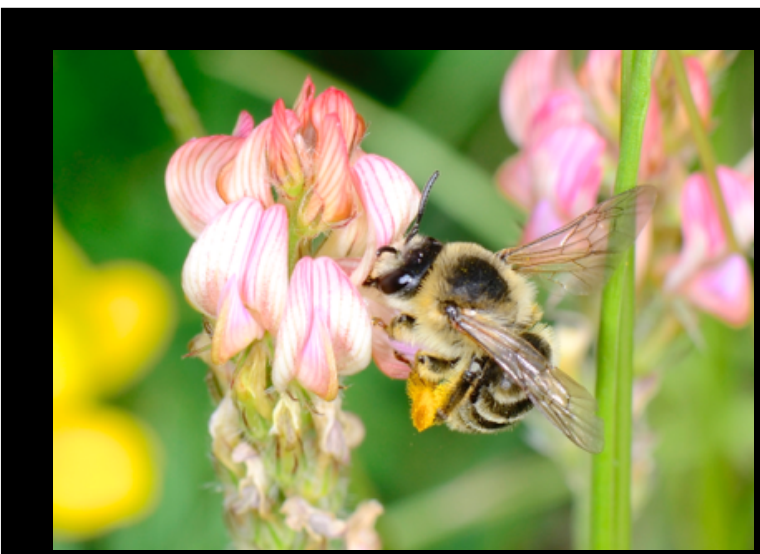

1. Melitta dimidiata femelle (Melittidae) sur Onobrychis sp. (Fabaceae)

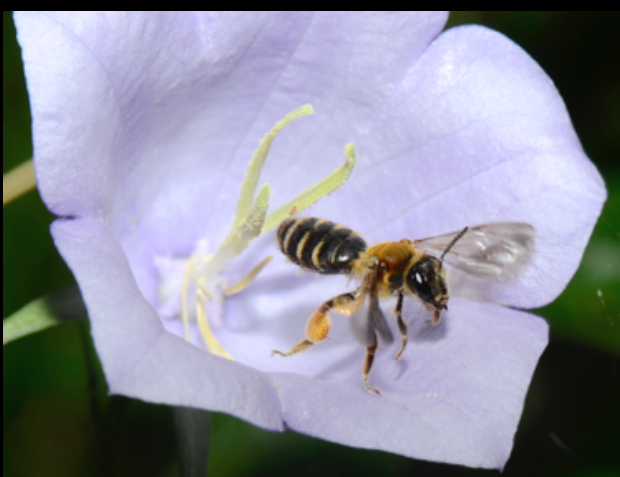

2. Andrena curvungula femelle (Andrenidae) sur Campanula persicifolia (Campanulaceae)

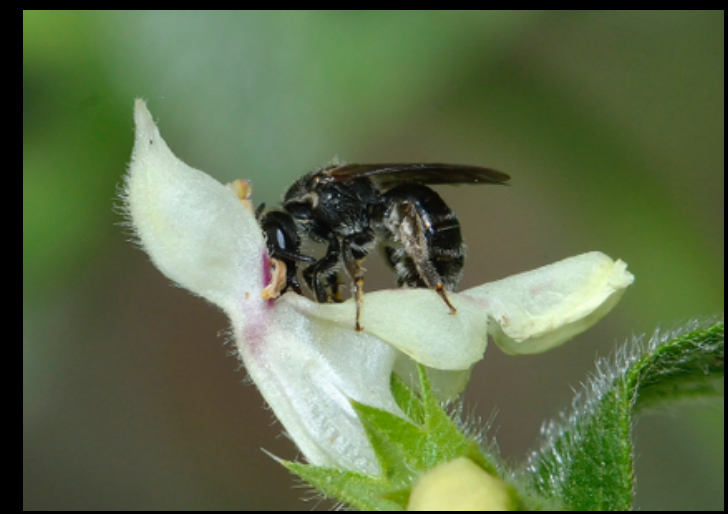

3. Lasioglossum clypeare femelle (Halictidae) sur Stachys recta (Lamiaceae)

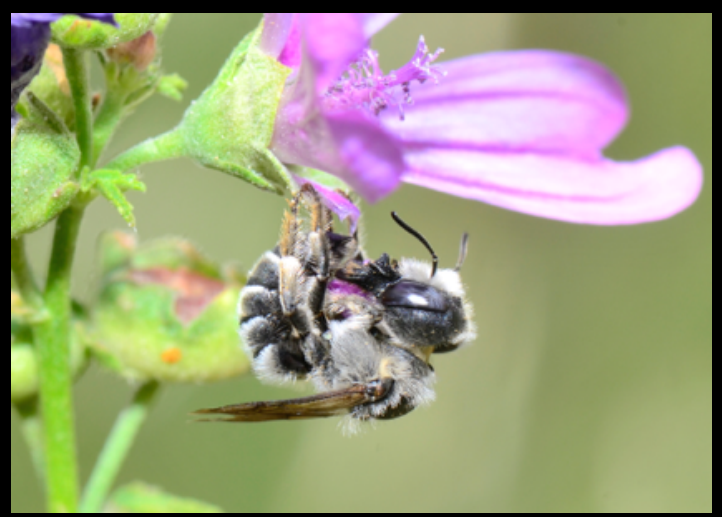

5. Hoplitis cristatula femelle (Megachilidae) sur Malva sylvestris (Malvaceae)

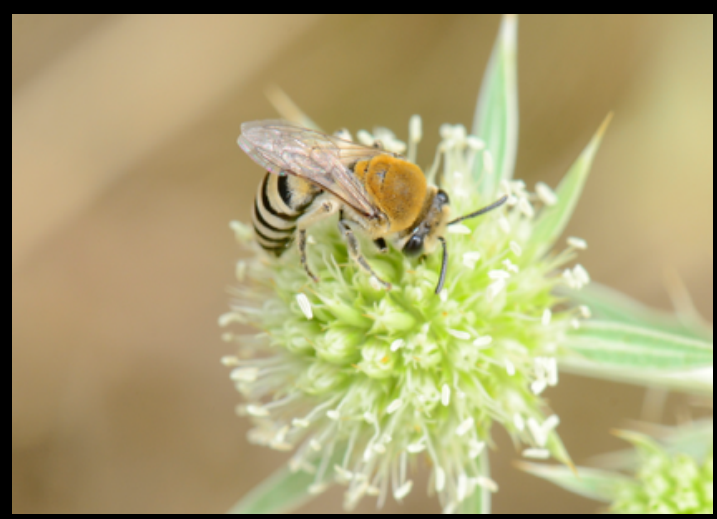

7. Colletes hylaeiformis femelle (Colletidae) sur Eryngium campestre (Apiaceae)

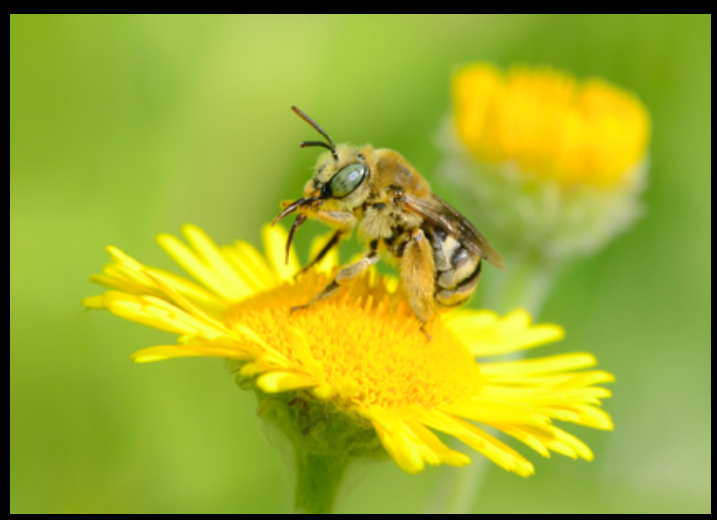

4. Tetraloniella alticincta femelle (Apidae) sur Pulicaria dysenterica (Asteraceae)

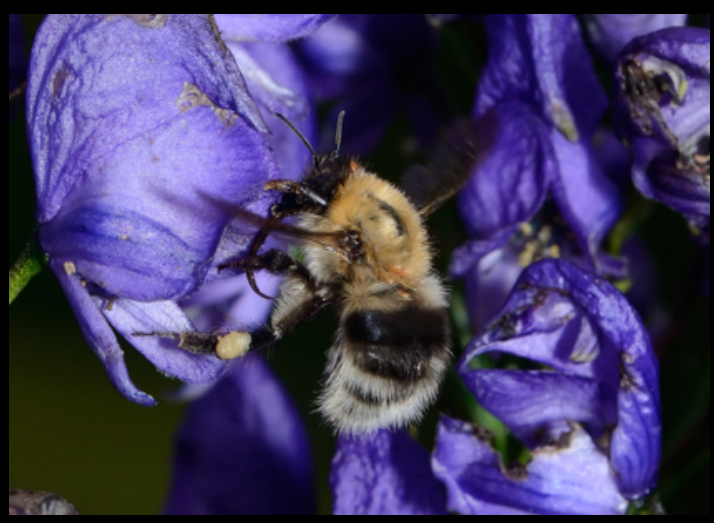

6. Bombus gerstaeckeri femelle (Apidae) sur Aconitum napellus (Ranunculaceae)

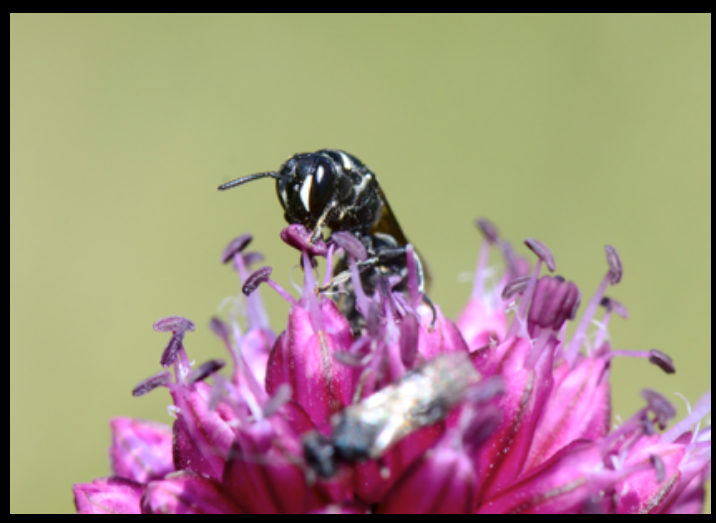

8. Hylaeus punctulatissimus femelle (Colletidae) sur Allium sphaerocephalon (Liliaceae) 